\title{
Asymmetric Synthesis of ent-Fissistigmatin C
}

Dongyang Xu, ${ }^{\S, 1}$ Jiadong Hu, ${ }^{\S, 2}$ Le Chen ${ }^{1}$, Lu Chen ${ }^{1}$, Jiang $\mathrm{Su}^{1}$, Jinjin

$$
\text { Yang }^{1} \text {, Siyu Deng }{ }^{1} \text {, Hongli Zhang }{ }^{*, 3} \text { and Weiqing Xie }{ }^{*, 1,4}
$$

${ }^{1}$ Shaanxi Key Laboratory of Natural Products \& Chemical Biology, College of Chemistry \& Pharmacy, Northwest A\&F University, 22 Xinong Road, Yangling 712100, Shaanxi, China

${ }^{2}$ School of Medicinal and Chemical Engineering, Yangling Vocational \& Technical College, 10 Xinong Road, Yangling 712100, Shaanxi, China

${ }^{3}$ State Key Laboratory of Stress Biology for Arid Areas, Northwest A\&F University, Yangling, Shaanxi, China

${ }^{4}$ Key Laboratory of Botanical Pesticide R\&D in Shaanxi Province, Yangling, Shaanxi 712100, China

${ }^{\S}$ D.X. and J.H. contributed equally

${ }^{*}$ Corresponding authors

*Email: xiewq@nwafu.edu.cn.

*Email: honglizhang@126.com.

\section{Contents}

1. General Information .1

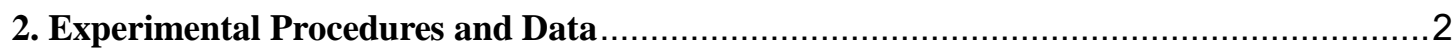

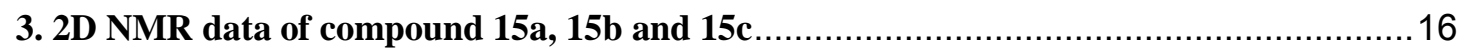

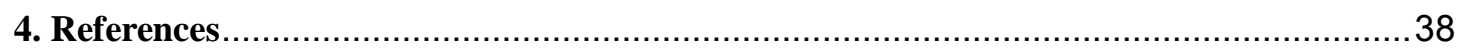

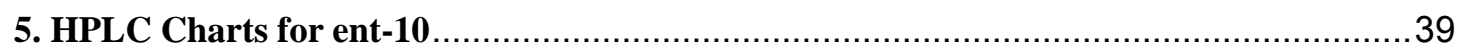

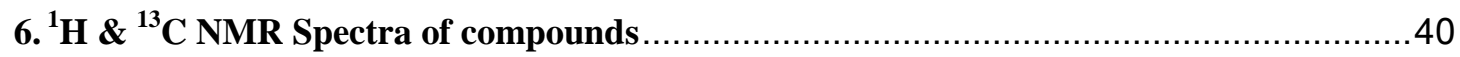

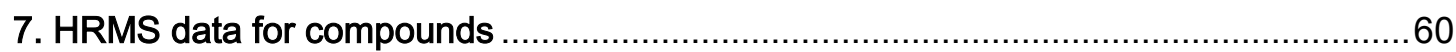




\section{General Information}

Unless otherwise noted, reagents were obtained from commercial sources and used without further purification. Non-aqueous reaction were conducted under an inert atmosphere of argon in flame-dried glassware. Anhydrous solvent were treated as follow: tetrahydrofuran and diethyl ether were distilled from sodium under argon atmosphere, dichcloromethane and toluene was distilled from calciumhydride under argon atmosphere. Anhydrous chloroform, acetonitrile, 1,2-dichloroethane, methanol and ethyl acetate were commercial available (Adamas, SafeDry, with molecular sieves).

Thin layer chromatography was conducted on Merck 60 F254 pre-coated silica gel plates. Column chromatography was carried out by normal silica gel (40-60 $\mu \mathrm{m}, 200-400$ mesh, Silicycle P60). NMR data including ${ }^{1} \mathrm{H}$ NMR and ${ }^{13} \mathrm{C}$ NMR spectra were recorded on Bruker AVANCE III 500MHz. The chemical shifts $(\delta)$ for ${ }^{1} \mathrm{H}$ and ${ }^{13} \mathrm{C}$ are given in ppm relative to residual signals of the solvents $\left(\mathrm{CHCl}_{3}\right.$ @ 7.26 ppm ${ }^{1} \mathrm{H}$ NMR, 77.16 ppm ${ }^{13} \mathrm{C}$ NMR). Coupling constants are given in $\mathrm{Hz}$.

Low mass spectra were measured on a Shimadzu LCMS-2010EV mass spectrometer (ESI). High resolution mass spectra were obtained from IonSpec 4.7 Tesla FTMS mass spectrometer (MALDI), Bruker APEXIII 7.0 TESLA FTMS (ESI)

Optical rotations were measured on a Anton Paar MCP 300 polarimeter and are reported as follows: $[\alpha]_{\mathrm{D}}^{\mathrm{rt}}(\mathrm{C}$ in $\mathrm{g} / 100 \mathrm{~mL}$, solvent). 


\section{Experimental Procedures and Data}

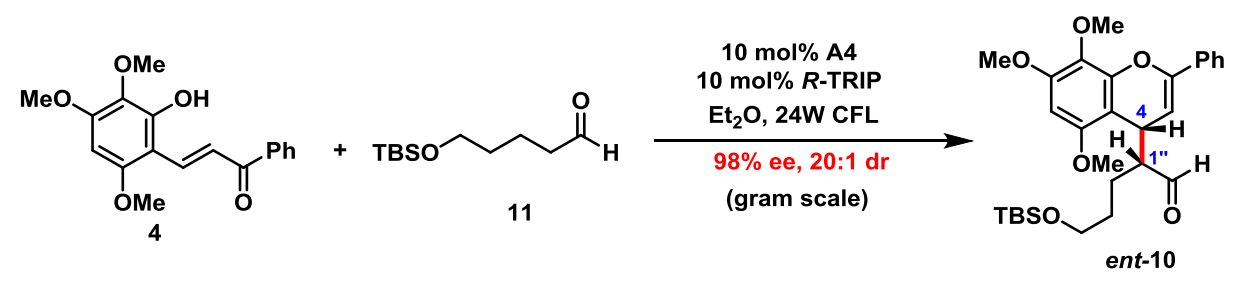

This compound was prepared according to our previous work ${ }^{1}$. A $250 \mathrm{~mL}$ flask was charged with the A4 (10 mol\%), R-TRIP (10 mol\%), 2-hydroxychalcone 4 (10 mmol), and corresponding aldehyde $11(15 \mathrm{mmol})$ dissolved in $\mathrm{Et}_{2} \mathrm{O}(100 \mathrm{~mL})$. After that, the vial was sealed and positioned approximately $5 \mathrm{~cm}$ away from the light source. A household full spectrum $24 \mathrm{~W}$ compact fluorescent light (CFL) bulb was used for irradiating the reaction mixture. A fan was used above the reaction apparatus for insuring the reaction temperature at RT. The reaction mixture was stirred at this condition until all 2-hydroxychalcone $\mathbf{4}$ was consumed as judged by TLC. The solvent was removed under vacuum. The residue was purified by flash column chromatography (ethyl acetate/petroleum ether $=1 / 10, \mathrm{v} / \mathrm{v}$ ) to afford the product $(4.49 \mathrm{~g}, 90 \%$ yield, $98 \% e e$, d.r. $>20: 1)$ as a pale-yellow oil. $\mathrm{R}_{\mathrm{f}}=0.51$ (ethyl acetate/petroleum ether $=1 / 5, \mathrm{v} / \mathrm{v})$.

${ }^{1} \mathbf{H}$ NMR $\left(\mathbf{5 0 0} \mathbf{~ M H z}, \mathbf{C D C l}_{3}\right) \delta 9.83(\mathrm{~d}, J=1.6 \mathrm{~Hz}, 1 \mathrm{H}), 7.70(\mathrm{~d}, J=7.3 \mathrm{~Hz}, 2 \mathrm{H}), 7.38(\mathrm{t}, J=7.4 \mathrm{~Hz}$, 2H), 7.37-7.30 (m, 1H), $6.25(\mathrm{~s}, 1 \mathrm{H}), 5.42(\mathrm{~d}, J=5.4 \mathrm{~Hz}, 1 \mathrm{H}), 4.36-4.20(\mathrm{~m}, 1 \mathrm{H}), 3.90(\mathrm{~s}, 3 \mathrm{H}), 3.89(\mathrm{~s}$, $3 \mathrm{H}), 3.82(\mathrm{~s}, 3 \mathrm{H}), 3.53-3.39(\mathrm{~m}, 1 \mathrm{H}), 2.90-2.78(\mathrm{~m}, 1 \mathrm{H}), 1.81-1.71(\mathrm{~m}, 1 \mathrm{H}), 1.57-1.49(\mathrm{~m}, 1 \mathrm{H}), 1.48-$ $1.40(\mathrm{~m}, 1 \mathrm{H}), 1.38-1.29(\mathrm{~m}, 1 \mathrm{H}), 0.80(\mathrm{~s}, 9 \mathrm{H}),-0.06(\mathrm{~d}, J=4.2 \mathrm{~Hz}, 6 \mathrm{H})$.

${ }^{13} \mathbf{C}$ NMR (126 MHz, $\left.\mathbf{C D C l}_{3}\right)$ : 204.6, 152.4, 152.3, 150.6, 147.23, 133.9, 131.7, 128.8, 128.5, 124.8, 104.0, 97.4, 91.7, 62.9, 61.5, 57.2, 56.6, 55.7, 31.1, 30.8, 26.0, 21.4, 18.3, -5.3, -5.2.

HRMS (ESI) $\mathrm{m} / \mathrm{z}$ : $[\mathrm{M}+\mathrm{H}]^{+}$Calcd for $\mathrm{C}_{29} \mathrm{H}_{39} \mathrm{O}_{6} \mathrm{Si}$ 511.2510; Found 511.2509.

NOTE: The A4 and R-TRIP could be recycled for 10 times without noticeable loss of reactivity for the $10 \mathrm{mmol}$ scale synthesis of ent-10.

Table S1. The Statistical Data of the Organocatalysts Recycled for the Synthesis of ent-10 ${ }^{a}$.

\begin{tabular}{lllllllllllll}
\hline Times & $1^{\text {st }}$ & $2^{\text {nd }}$ & $3^{\text {rd }}$ & $4^{\text {th }}$ & $5^{\text {th }}$ & $6^{\text {th }}$ & $7^{\text {th }}$ & $8^{\text {th }}$ & $9^{\text {th }}$ & $10^{\text {th }}$ & $11^{\text {th }}$ & $12^{\text {th }}$ \\
\hline scale (mmol) & 10 & 10 & 10 & 15 & 10 & 10 & 10 & 10 & 10 & 10 & 10 & 10 \\
t (d) & 3 & 3 & 3 & 5 & 3 & 4 & 4 & 4 & 4 & 5 & 5 & 5 \\
yield (\%) & 93 & 93 & 92 & 90 & 92 & 91 & 92 & 90 & 90 & 90 & 91 & 90 \\
$\boldsymbol{e} \boldsymbol{e}(\boldsymbol{\%})$ & 98 & 98 & 98 & 98 & 98 & 98 & 98 & 98 & 98 & 98 & 98 & 98 \\
\hline
\end{tabular}

a $\boldsymbol{d r}>20: 1$ for all tests

$[\alpha]_{\mathrm{D}}^{25.0}=+88.5\left(c 0.265, \mathrm{CHCl}_{3}\right)$.

Enantiomeric excess was found to be $98 \%$ by chiral HPLC (ChiralPak OD-H column, hexane $/ i$-PrOH $=$ 9:1, $\left.214 \mathrm{~nm}, 0.7 \mathrm{~mL} / \mathrm{min}, \mathrm{t}_{\text {major }}=9.00 \mathrm{~min}, \mathrm{t}_{\text {minor }}=13.46 \mathrm{~min}\right)$.
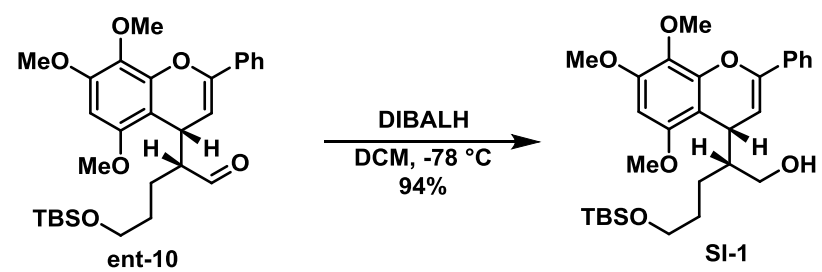

To a solution of ent-10 (4.44 g, $8.7 \mathrm{mmol}, 1.0$ equiv.) in dichloromethane $20 \mathrm{~mL}$ at $-78{ }^{\circ} \mathrm{C}$ under argon atomosphere was added DIBAL-H (12 mL, $1.5 \mathrm{M}$ in Toluene, 2.0 equiv.) dropwise. The resulting mixture was stirred at this temperature until ent-10 were consumed completely as judged by 
TLC (about $30 \mathrm{~min}$ ). Then excess DIBAL-H was destroyed by addition of water dropwise, and the resulting mixture was poured into an ice-cold 5\% aqueous solution of tartaric acid. The mixture was extracted with EtOAc and washed with saturated $\mathrm{NaHCO}_{3}$ and brine, the combined organic layers were dried $\left(\mathrm{Na}_{2} \mathrm{SO}_{4}\right)$ and evaporated. The residue was purified by flash column chromatography (ethyl acetate/petroleum ether $=1 / 5, \mathrm{v} / \mathrm{v})$ to afford the corresponding alcohol SI-1 (4.2 g, 94\%yield) as a colorless oil. $[\alpha]_{\mathrm{D}}{ }^{25.0}=+76.54\left(c 0.105, \mathrm{CHCl}_{3}\right) . \mathrm{R}_{\mathrm{f}}=0.32($ ethyl acetate/petroleum ether $=1 / 3, \mathrm{v} / \mathrm{v})$

${ }^{1}$ H NMR (500 MHz, CDCl $) \delta 7.74(\mathrm{~d}, J=7.3 \mathrm{~Hz}, 2 \mathrm{H}), 7.41-7.35(\mathrm{~m}, 2 \mathrm{H}), 7.34-7.29(\mathrm{~m}, 1 \mathrm{H}), 6.26(\mathrm{~s}$, $1 \mathrm{H}), 5.68(\mathrm{~d}, J=5.8 \mathrm{~Hz}, 1 \mathrm{H}), 3.98(\mathrm{t}, J=5.3 \mathrm{~Hz}, 1 \mathrm{H}), 3.90(\mathrm{~s}, 3 \mathrm{H}), 3.89(\mathrm{~s}, 3 \mathrm{H}), 3.82(\mathrm{~s}, 3 \mathrm{H}), 3.73-3.62$ $(\mathrm{m}, 2 \mathrm{H}), 3.46(\mathrm{t}, J=6.1 \mathrm{~Hz}, 1 \mathrm{H}), 2.16(\mathrm{~s}, 1 \mathrm{H}), 2.01-1.88(\mathrm{~m}, 1 \mathrm{H}), 1.56-1.20(\mathrm{~m}, 4 \mathrm{H}), 0.81(\mathrm{~s}, 9 \mathrm{H}),-0.04$ (d, $J=4.8 \mathrm{~Hz}, 6 \mathrm{H})$.

${ }^{13}$ C NMR (126 MHz, $\left.\mathbf{C D C l}_{3}\right) \delta 152.4,151.8,149.9,147.4,134.2,131.7,128.5,128.4,124.7,106.6$, $99.4,91.8,63.8,63.5,61.5,56.5,55.9,46.5,31.6,30.8,26.0,23.6,18.3,-5.3,-5.2$.

HRMS (ESI) m/z: $[\mathrm{M}+\mathrm{H}]^{+}$Calcd for $\mathrm{C}_{29} \mathrm{H}_{43} \mathrm{O}_{6} \mathrm{Si}$ 515.2823; Found 515.2823.
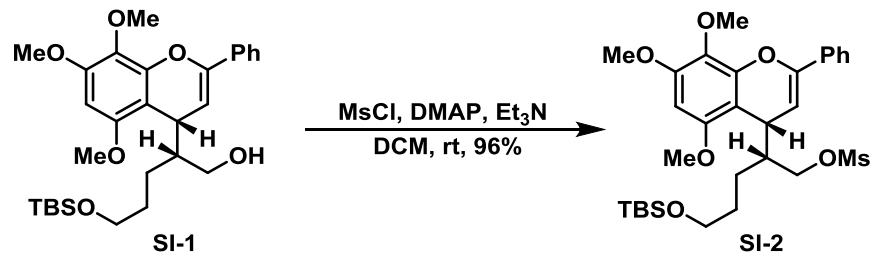

To a solution of SI-1 (3.036 g, $5.9 \mathrm{mmol}, 1.0$ equiv) in dry dichloromethane $(20 \mathrm{~mL})$ at $0{ }^{\circ} \mathrm{C}$ were added $\mathrm{Et}_{3} \mathrm{~N}$ (1.7 mL, $12 \mathrm{mmol}, 2.0$ equiv), methanesulfonyl chloride ( $0.7 \mathrm{~mL}, 8.8 \mathrm{mmol}, 1.5$ equiv), and DMAP (cat.). The resulting mixture was stirred at room temperature until SI-1 were consumed completely as judged by TLC. The reaction was quenched with $1 \mathrm{~N} \mathrm{HCl}$ and extracted with dichloromethane $(3 \times 15 \mathrm{~mL})$. The organic fractions were collected, dried over $\mathrm{Na}_{2} \mathrm{SO}_{4}$, and filtered, and the solvent was removed in vacuo. The residue was purified by flash column chromatography (ethyl acetate/petroleum ether $=1 / 10, \mathrm{v} / \mathrm{v}$ ) to afford the corresponding SI-2 (3.36 g, 96\%yield) as a pale-yellow oil. $[\alpha]_{\mathrm{D}}{ }^{25.0}=+80.03\left(c 0.12, \mathrm{CHCl}_{3}\right) . \mathrm{R}_{\mathrm{f}}=0.61($ ethyl acetate/petroleum ether $=1 / 3, \mathrm{v} / \mathrm{v})$

${ }^{1} \mathbf{H}$ NMR (500 MHz, $\left.\mathbf{C D C l}_{3}\right) \delta$ 7.78-7.70 (m, 2H), 7.47-7.36 (m, 2H), 7.35-7.32 (m, 1H), $6.25(\mathrm{~s}, 1 \mathrm{H})$, $5.58(\mathrm{~d}, J=5.7 \mathrm{~Hz}, 1 \mathrm{H}), 4.40-4.24(\mathrm{~m}, 2 \mathrm{H}), 4.09-4.01(\mathrm{~m}, 1 \mathrm{H}), 3.90(\mathrm{~s}, 3 \mathrm{H}), 3.89(\mathrm{~s}, 3 \mathrm{H}), 3.81(\mathrm{~s}, 3 \mathrm{H})$, $3.41(\mathrm{t}, J=6.0 \mathrm{~Hz}, 2 \mathrm{H}), 3.07(\mathrm{~s}, 3 \mathrm{H}), 2.38-2.26(\mathrm{~m}, 1 \mathrm{H}), 1.49-1.41(\mathrm{~m}, 1 \mathrm{H}), 1.37-1.20(\mathrm{~m}, 3 \mathrm{H}), 0.78$ (s, 9H), $-0.07(\mathrm{~d}, J=3.5 \mathrm{~Hz}, 6 \mathrm{H})$.

${ }^{13}$ C NMR (126 MHz, $\left.\mathbf{C D C l}_{3}\right) \delta 152.5,152.1,150.5,147.3,133.9,131.5,128.6,128.5,124.7,105.0$, 97.1, 91.6, 71.1, 63.0, 61.5, 56.5, 55.7, 42.8, 37.6, 31.6, 30.6, 26.0, 23.1, 18.3, -5.4, -5.3.

HRMS (ESI) m/z: $[\mathrm{M}+\mathrm{H}]^{+}$Calcd for $\mathrm{C}_{30} \mathrm{H}_{45} \mathrm{O}_{8} \mathrm{SSi}$ 593.2599; Found 593.2593
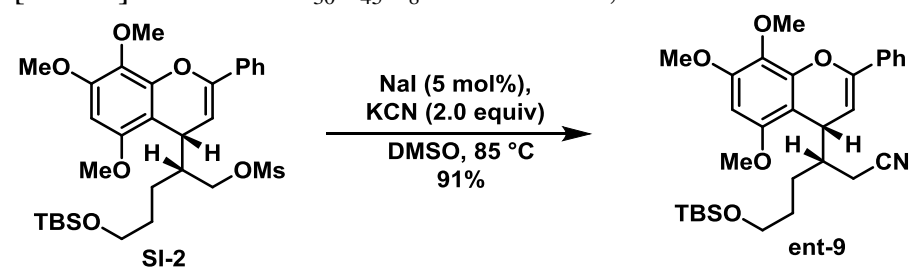

To a solution of SI-2 (3.36 g, $5.7 \mathrm{mmol}, 1.0$ equiv) in DMSO (20 mL) at room temperature were added $\mathrm{KCN}$ (740 mg, $11.4 \mathrm{mmol}, 2.0$ equiv), NaI (43 mg, $0.28 \mathrm{mmol}, 0.05$ equiv). After the resulting mixture was stirred at $85{ }^{\circ} \mathrm{C}$ for 5 hours. The reaction was cooled to room temperature and quenched with water and extracted with ethyl acetate $(3 \times 20 \mathrm{~mL})$. The organic fractions were collected, and washed with brine $(3 \times 10 \mathrm{~mL})$, dried over $\mathrm{Na}_{2} \mathrm{SO}_{4}$, and filtered, concentrated. The residue was purified by flash column chromatography (ethyl acetate/petroleum ether $=1 / 15, \mathrm{v} / \mathrm{v}$ ) to afford the corresponding ent-9 $\left(2.72 \mathrm{~g}, 91 \%\right.$ yield) as a yellow oil. $[\alpha]_{\mathrm{D}}^{25.0}=+56.38\left(c 0.1125, \mathrm{CHCl}_{3}\right) . \mathrm{R}_{\mathrm{f}}=0.60$ (ethyl acetate/petroleum ether $=1 / 5, \mathrm{v} / \mathrm{v})$

${ }^{1} \mathbf{H}$ NMR (500 MHz, $\left.\mathbf{C D C l}_{3}\right) \delta 7.75(\mathrm{~d}, J=7.3 \mathrm{~Hz}, 2 \mathrm{H}), 7.44-7.35(\mathrm{~m}, 2 \mathrm{H}), 7.37-7.31(\mathrm{~m}, 1 \mathrm{H}), 6.26(\mathrm{~s}$, $1 \mathrm{H}), 5.61(\mathrm{~d}, J=5.7 \mathrm{~Hz}, 1 \mathrm{H}), 4.04-3.93(\mathrm{~m}, 1 \mathrm{H}), 3.89$ (s, 6H), $3.82(\mathrm{~s}, 3 \mathrm{H}), 3.61-3.35$ (m, 2H), 2.56- 

$\mathrm{Hz}, 6 \mathrm{H})$.

${ }^{13} \mathbf{C}$ NMR (126 MHz, $\left.\mathbf{C D C l}_{3}\right) \delta 152.4,152.2,150.8,147.1,133.8,131.5,128.6,128.4,124.7,119.3$, 104.9, 97.0, 91.7, 62.8, 61.4, 56.4, 55.6, 40.6, 34.0, 30.3, 26.0, 25.9, 19.8, 18.2, -5.5, -5.4.

HRMS (ESI) m/z: $[\mathrm{M}+\mathrm{H}]^{+}$Calcd for $\mathrm{C}_{30} \mathrm{H}_{42} \mathrm{NO}_{5} \mathrm{Si}$ 524.2827; Found 524.2827.

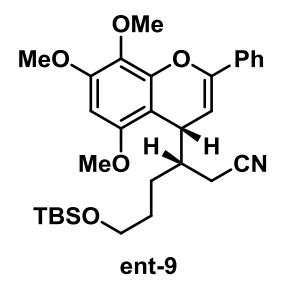
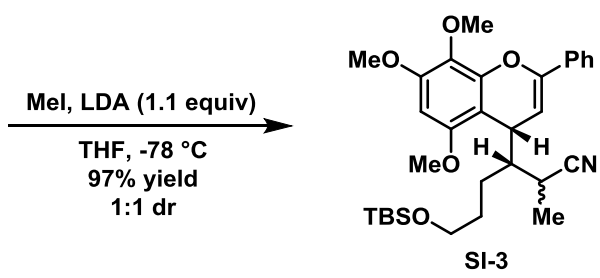

A solution of $n$ - $\mathrm{BuLi}$ (2.5 M in hexane, $2.68 \mathrm{~mL}, 6.7 \mathrm{mmol}, 1.3$ equiv) was added dropwise to a solution of $i-\mathrm{Pr}_{2} \mathrm{NH}\left(0.94 \mathrm{~mL}, 6.7 \mathrm{mmol}, 1.3\right.$ equiv) in dry $\mathrm{THF}(15 \mathrm{~mL})$ at $0{ }^{\circ} \mathrm{C}$ under argon atomosphere. The LDA solution was stirred for $0.5 \mathrm{~h}$ at this temperature, then cooled to $-78{ }^{\circ} \mathrm{C}$. Now, a solution of nitrile ent-9 $(2.72 \mathrm{~g}, 5.2 \mathrm{mmol})$ in THF $(5 \mathrm{~mL})$ was added dropwise within $10 \mathrm{~min}$. A yellowish color of the nitrile anion appeared within minutes. The mixture was stirred for $1 \mathrm{~h}$ at $-78{ }^{\circ} \mathrm{C}$ before MeI $(0.38 \mathrm{~mL}, 6.2 \mathrm{mmol}, 1.2$ equiv) was introduced at this temperature. The mixture was stirred for $30 \mathrm{~min}$ at $-78^{\circ} \mathrm{C}$. Then the mixture was treated with saturated $\mathrm{NH}_{4} \mathrm{Cl}$ solution $(25 \mathrm{~mL})$ and warmed to room temperature. The mixture was extracted with ethyl acetate $(3 \times 40 \mathrm{~mL})$. The combined organic extracts were washed with saturated brine, dried over $\mathrm{Na}_{2} \mathrm{SO}_{4}$, filtered, and concentrated, and the residue purified by flash chromatography (ethyl acetate/petroleum ether $=1 / 30, \mathrm{v} / \mathrm{v}$ ) to afford the corresponding SI-3 (2.71 g, 97\%yield, $\mathrm{dr}=1: 1)$ as a yellow oil. This diastereomers was no consequence for the subsequent reaction.

\section{Synthesis of compound 12}

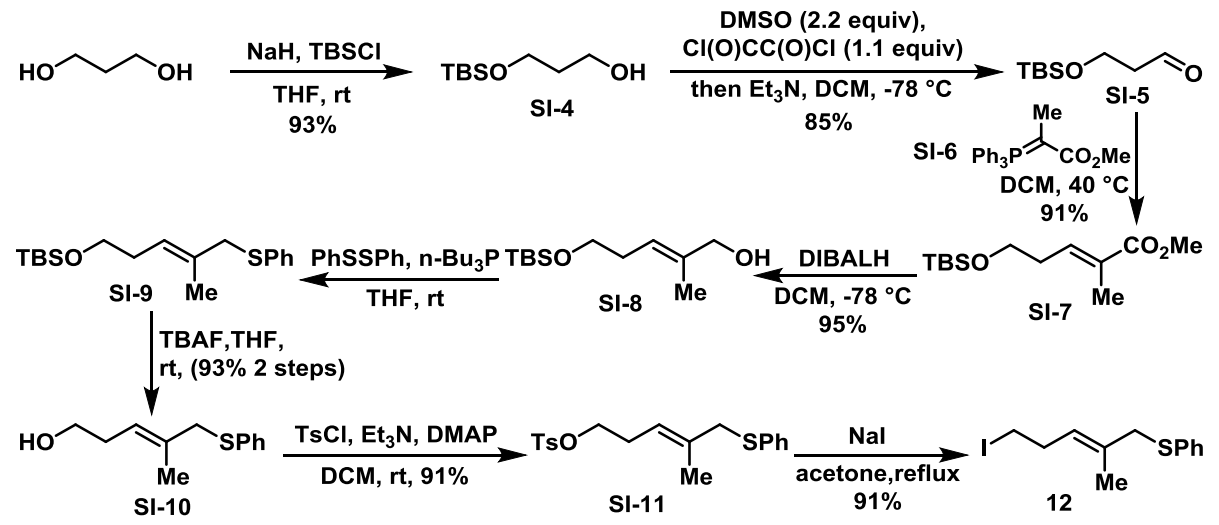

To a solution of 1,3-propanediol ( $7.2 \mathrm{~mL}, 100 \mathrm{mmol}, 1.0$ equiv) in anhydrous THF (200 mL) was added $\mathrm{NaH}\left(60 \%\right.$ suspension in mineral oil, $4 \mathrm{~g}, 100 \mathrm{mmol}, 1.0$ equiv) in several portions at $0{ }^{\circ} \mathrm{C}$. The resulting mixture was allowed to warm up to room temperature and stirred for an additional $45 \mathrm{~min}$ before a solution of TBSCl (15 g, $100 \mathrm{mmol}, 1.0$ equiv) in anhydrous THF (100 mL) was added via a cannula. After $3 \mathrm{~h}$, TLC analysis indicated the reaction was complete. The reaction mixture was quenched with water $(100 \mathrm{~mL})$ and extracted with ether $(3 \times 100 \mathrm{~mL})$. The combined organic layers were washed with sat aq $\mathrm{NaHCO}_{3}(100 \mathrm{~mL})$ and sat aq $\mathrm{NaCl}(100 \mathrm{~mL})$, dried over $\mathrm{Na}_{2} \mathrm{SO}_{4}$, filtered, and concentrated in vacuo. The crude product was purified by flash column chromatography (ethyl acetate/petroleum ether $=1 / 10, \mathrm{v} / \mathrm{v})$ to afford the desired mono-TBS protected alcohol SI-4 $(17.7 \mathrm{~g}, 95 \%$ yield) as colorless oil. $R_{\mathrm{f}}=0.40$ (ethyl acetate/petroleum ether $=1 / 3$, v/v) 
To a solution of Oxalyl Chloride $\left(4.3 \mathrm{~mL}, 55 \mathrm{mmol}, 1.1\right.$ equiv) in DCM $(100 \mathrm{~mL})$ at $-78^{\circ} \mathrm{C}$ under argon atomosphere was added DMSO $(7.8 \mathrm{~mL}, 110 \mathrm{mmol}, 2.2$ equiv) dropwise. The reaction mixture was stirred at this temperature for $30 \mathrm{~min}$, then to the resulting solution was added mono-protected alcohol SI-4 (9.52 g, $50 \mathrm{mmol}, 1.0$ equiv) slowly in DCM $(50 \mathrm{~mL})$. The reaction mixture was stirred at $-78^{\circ} \mathrm{C}$ for $1 \mathrm{~h}$, then $\mathrm{Et}_{3} \mathrm{~N}(35 \mathrm{~mL}, 250 \mathrm{mmol}, 5.0$ equiv) was added and the reaction mixture was stirred same temperature for $10 \mathrm{~min}$ before being warm up to room temperature. When TLC analysis showed the disappearance of the starting material, the reaction was quenched with water $(100 \mathrm{~mL})$ and extracted with DCM $(3 \times 150 \mathrm{~mL})$. The combined organic layers were washed with water $(2 \times 100 \mathrm{~mL})$ and sat aq $\mathrm{NaCl}(200 \mathrm{~mL})$, dried over $\mathrm{Na} 2 \mathrm{SO} 4$, filtered, and concentrated in vасио. The crude aldehyde was purified by flash column chromatography (ethyl acetate/petroleum ether $=1 / 20, \mathrm{v} / \mathrm{v})$ to afford the desired aldehyde SI-5 $(8.05 \mathrm{~g}, 85 \%$ yield $)$ as colorless oil. $\mathrm{R}_{\mathrm{f}}=0.40$ (ethyl acetate/petroleum ether $=1 / 5, \mathrm{v} / \mathrm{v})$

To a solution of the aldehyde SI-5 (4.52 g, $24 \mathrm{mmol}, 1.0$ equiv) in DCM (60 mL) was added SI-6 ${ }^{2}$ (20.6 g, 60 mmol, 2.5 equiv) in several portions at rt. The resulting mixture was refluxed for $6 \mathrm{~h}$, then cooled down to rt and concentrated in vacuo. The crude mixture was purified using silica gel flash column chromatography (ethyl acetate/petroleum ether $=1 / 30, \mathrm{v} / \mathrm{v}$ ) to provide the desired unsaturated ester SI-7 (5.68 g, 91\% yield) as an inseparable 20:1 (E/Z) mixture. $\mathrm{R}_{\mathrm{f}}=0.6$ (ethyl acetate/petroleum ether $=1 / 8, \mathrm{v} / \mathrm{v})$

${ }^{1}$ H NMR (500 MHz, CDCl $)$ ) 6.84-6.71 (m, 1H), $3.72(\mathrm{~s}, 3 \mathrm{H}), 3.69(\mathrm{t}, J=6.7 \mathrm{~Hz}, 2 \mathrm{H}), 2.46-2.31(\mathrm{~m}$, $2 \mathrm{H}), 1.84(\mathrm{~s}, 3 \mathrm{H}), 0.88(\mathrm{~s}, 9 \mathrm{H}), 0.04(\mathrm{~s}, 6 \mathrm{H})$.

${ }^{13}$ C NMR (126 MHz, $\mathbf{C D C l}_{3}$ ) $\delta$ 168., 139.0, 129.2, 61.9, 51.8, 32.6, 26.0, 18.4, 12.7, -5.2.

HRMS (ESI) m/z: $[\mathrm{M}+\mathrm{H}]^{+}$Calcd for $\mathrm{C}_{13} \mathrm{H}_{27} \mathrm{O}_{3} \mathrm{Si} 259.1724$; Found 259.1724.

To a solution of the aforementioned 20:1 (E:Z) mixture of unsaturated ester $(5.68 \mathrm{~g}, 22 \mathrm{mmol}$, 1.0 equiv) in anhydrous $\mathrm{CH}_{2} \mathrm{Cl}_{2}(50 \mathrm{~mL})$ was added slowly DIBAL-H (32 mL, $1.5 \mathrm{M}$ solution in Toluene, $48.4 \mathrm{mmol}, 2.2$ equiv) at $-78{ }^{\circ} \mathrm{C}$. The reaction was stirred at $-78^{\circ} \mathrm{C}$ for $2 \mathrm{~h}$ and the TLC analysis indicated the complete conversion from the starting material to the product. The reaction was quenched with water $(20 \mathrm{~mL})$ at $-78{ }^{\circ} \mathrm{C}$ carefully, and the resulting mixture warm up to room temperature and poured into an ice-cold $5 \%$ aqueous solution of tartaric acid. The mixture was extracted with DCM and washed with saturated $\mathrm{NaHCO}_{3}$ and brine, the combined organic layers were dried $\left(\mathrm{Na}_{2} \mathrm{SO}_{4}\right)$ and evaporated. The residue was purified by flash column chromatography (ethyl acetate/petroleum ether $=1 / 10, \mathrm{v} / \mathrm{v})$ to afford the corresponding alcohol SI-8 (4.8 g, 95\%yield) as a colorless oil. $\mathrm{R}_{\mathrm{f}}=0.40$ (ethyl acetate/petroleum ether $=1 / 8, \mathrm{v} / \mathrm{v}$ )

${ }^{1} \mathbf{H}$ NMR (500 MHz, CDCl $) \delta$ 5.43-5.34 (m, 1H), $3.95(\mathrm{~s}, 2 \mathrm{H}), 3.58(\mathrm{t}, J=7.0 \mathrm{~Hz}, 2 \mathrm{H}), 2.24(\mathrm{q}, J=$ $7.1 \mathrm{~Hz}, 2 \mathrm{H}), 2.13(\mathrm{~s}, 1 \mathrm{H}), 1.64(\mathrm{~s}, 3 \mathrm{H}), 0.86(\mathrm{~s}, 9 \mathrm{H}), 0.02(\mathrm{~s}, 6 \mathrm{H})$.

${ }^{13}$ C NMR (126 MHz, $\mathbf{C D C l}_{3}$ ) $\delta$ 136.8, 122.0, 68.7, 62.9, 31.5, 26.0, 18.4, 13.8, -5.17.

HRMS (ESI) m/z: [M + H]+ Calcd for $\mathrm{C}_{12} \mathrm{H}_{27} \mathrm{O}_{2} \mathrm{Si} 231.1775$; Found 231.1772.

To a solution of alcohol SI-8 (4.62 g, 20 mmol, 1.0 equiv) and diphenyldisulfide (13 g, $60 \mathrm{mmol}$, 3.0 equiv) in dry THF $(40 \mathrm{~mL})$ at ambient temperature under argon atomosphere was added Tributylphosphine ( $15 \mathrm{~mL}, 60 \mathrm{mmol}, 3.0$ equiv), and the resulting mixture was stirred for 5 hours, before water $(40 \mathrm{~mL})$ was added. The phases were separated and extracted with ethyl acetate $(3 \times 40$ $\mathrm{mL})$ and washed with $10 \% \mathrm{NaOH}(\mathrm{aq})(80 \mathrm{~mL})$ and brine, the combined organic layers were dried $\left(\mathrm{Na}_{2} \mathrm{SO}_{4}\right)$ and evaporated. The residue was used directly without further purification.

To a solution of crude Phenyl sulfide SI-9 in THF (40 mL) at room temperature was added TBAF (24 mL, 1.0 M in THF, $24 \mathrm{mmol}, 1.2$ equiv) slowly, and stirred at this temperature for 6 hours. Then water $(40 \mathrm{~mL})$ was added and extracted with ethyl acetate $(3 \times 40 \mathrm{~mL})$ and washed with brine, the combined organic layers were dried $\left(\mathrm{Na}_{2} \mathrm{SO}_{4}\right)$ and evaporated. The residue was purified by flash column chromatography (ethyl acetate/petroleum ether $=1 / 10, \mathrm{v} / \mathrm{v}$ ) to afford the corresponding alcohol 
SI-10 (3.87 g, 93\%yield over 2 steps) as a colorless oil. $\mathrm{R}_{\mathrm{f}}=0.5$ (ethyl acetate/petroleum ether $=1 / 5$, $\mathrm{v} / \mathrm{v})$

${ }^{1} \mathbf{H}$ NMR (500 MHz, $\left.\mathbf{C D C l}_{3}\right) \delta$ 7.35-7.30 (m, 2H), 7.30-7.23 (m, 2H), 7.21-7.16 (m, 1H), 5.18-5.11 (m, 1H), $3.49(\mathrm{~s}, 3 \mathrm{H}), 3.43(\mathrm{t}, J=6.5 \mathrm{~Hz}, 2 \mathrm{H}), 2.19(\mathrm{q}, J=6.8 \mathrm{~Hz}, 2 \mathrm{H}), 1.75(\mathrm{~s}, 3 \mathrm{H})$.

${ }^{13}$ C NMR (126 MHz, $\mathbf{C D C l}_{3}$ ) $\delta$ 135.9, 133.5, 131.2, 128.7, 126.6, 124.7, 62.0, 44.5, 31.6, 15.3.

HRMS (ESI) m/z: [M + H]+ Calcd for $\mathrm{C}_{12} \mathrm{H}_{17} \mathrm{OS} 209.0995$; Found 209.0994.

To a solution of SI-10 (3.83 g, $18.4 \mathrm{mmol}, 1.0$ equiv) in dry dichloromethane $(40 \mathrm{~mL})$ at $0{ }^{\circ} \mathrm{C}$ were added $\mathrm{Et}_{3} \mathrm{~N}$ (5.2 mL, $37 \mathrm{mmol}, 2.0$ equiv), 4-Toluolsulfonyl chloride (4.2 g, 22 mmol, 1.2 equiv), and DMAP $(225 \mathrm{mg})$. The resulting mixture was stirred at room temperature until SI-10 were consumed completely as judged by TLC. The reaction was quenched with $1 \mathrm{~N} \mathrm{HCl}$ and extracted with dichloromethane $(3 \times 40 \mathrm{~mL})$. The organic fractions were collected, dried over $\mathrm{Na}_{2} \mathrm{SO}_{4}$, and filtered, and the solvent was removed in vacuo. The residue was purified by flash column chromatography (ethyl acetate/petroleum ether $=1 / 10, \mathrm{v} / \mathrm{v})$ to afford $\mathbf{S I - 1 1}(6.07 \mathrm{~g}, 91 \%$ yield $)$ as a pale-yellow oil. $\mathbf{R}_{\mathrm{f}}=$ 0.6 (ethyl acetate/petroleum ether $=1 / 4, \mathrm{v} / \mathrm{v}$ )

${ }^{1}$ H NMR (500 MHz, $\left.\mathbf{C D C l}_{3}\right) \delta 7.76(\mathrm{~d}, J=8.3 \mathrm{~Hz}, 2 \mathrm{H}), 7.33(\mathrm{~d}, J=8.0 \mathrm{~Hz}, 2 \mathrm{H}), 7.31-7.27$ (m, 2H), 7.26-7.21 (m, 2H), 7.20-7.13 (m, 1H), 5.15-4.94 (m, 1H), $3.84(\mathrm{t}, J=7.0 \mathrm{~Hz}, 2 \mathrm{H}), 3.42(\mathrm{~s}, 2 \mathrm{H})$, $2.44(\mathrm{~s}, 3 \mathrm{H}), 2.30(\mathrm{q}, J=7.2 \mathrm{~Hz}, 2 \mathrm{H}), 1.69(\mathrm{~s}, 3 \mathrm{H})$.

${ }^{13}$ C NMR (126 MHz, $\mathbf{C D C l}_{3}$ ) $\delta 144.8,136.1,134.6,133.3,130.9,129.9,128.8,128.0,126.6,122.0$, 69.4, 44.2, 28.0, 21.8, 15.5 .

HRMS (ESI) m/z: $[\mathrm{M}+\mathrm{H}]^{+}$Calcd for $\mathrm{C}_{19} \mathrm{H}_{23} \mathrm{O}_{3} \mathrm{~S}_{2}$ 363.1083; Found 363.1081.

To a solution of sodium iodide $(7.7 \mathrm{~g}, 51 \mathrm{mmol}, 3.0$ equiv) in acetone $(30 \mathrm{~mL})$ was added a solution of tosylate SI-11 (6.178 g, $17 \mathrm{mmol}, 1.0$ equiv) in acetone $(20 \mathrm{~mL})$ at room temperature. The mixture was heated for $5 \mathrm{~h}$ under reflux. The reaction mixture was concentrated in vacuo. The mixture was poured into water $(40 \mathrm{~mL})$ and extracted three times with ethyl acetate. The combined organic layers were dried over $\mathrm{Na}_{2} \mathrm{SO}_{4}$ and concentrated in vacuo. The residue was purified by flash column chromatography (petroleum ether) to afford 12 (4.953 g, 91\%yield) as a yellow oil. $\mathrm{R}_{\mathrm{f}}=0.8$ (ethyl acetate/petroleum ether $=1 / 30, \mathrm{v} / \mathrm{v})$

${ }^{1} \mathbf{H}$ NMR (500 MHz, $\left.\mathbf{C D C l}_{3}\right) \delta$ 7.36-7.31 (m, 2H), 7.30-7.23 (m, 2H), 7.21-7.15 (m, 1H), 5.22-5.10 (m, 1H), $3.48(\mathrm{~s}, 2 \mathrm{H}), 2.96(\mathrm{t}, J=7.4 \mathrm{~Hz}, 2 \mathrm{H}), 2.54(\mathrm{q}, J=7.3 \mathrm{~Hz}, 2 \mathrm{H}), 1.74(\mathrm{~s}, 3 \mathrm{H})$.

${ }^{13}$ C NMR (126 MHz, $\mathbf{C D C l}_{3}$ ) $\delta$ 136.1, 133.4, 130.9, 128.8, 127.2, 126.6, 44.2, 32.5, 15.6, 4.9.

HRMS (ESI) m/z: [M + H] $]^{+}$Calcd for $\mathrm{C}_{12} \mathrm{H}_{16} \mathrm{IS} 319.0012$; Found 319.0009.
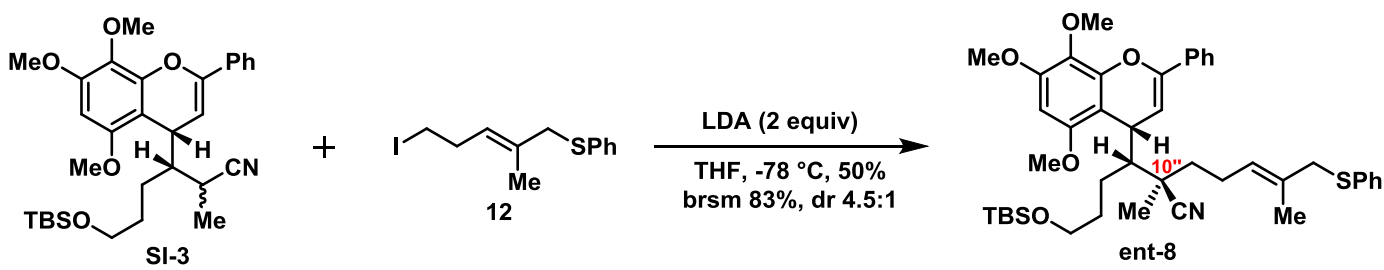

A solution of $n$-BuLi (2.5 M in hexane, $0.28 \mathrm{~mL}, 2.0 \mathrm{mmol}, 2.0$ equiv) was added dropwise to a solution of $i-\mathrm{Pr}_{2} \mathrm{NH}\left(0.8 \mathrm{~mL}, 2.0 \mathrm{mmol}, 2.0\right.$ equiv) in dry THF $(5 \mathrm{~mL})$ at $0{ }^{\circ} \mathrm{C}$ under argon atomosphere. The LDA solution was stirred for $0.5 \mathrm{~h}$ at this temperature, then cooled to $-78{ }^{\circ} \mathrm{C}$. Now, a solution of nitrile SI-3 (538 mg, $1.0 \mathrm{mmol}, 1.0$ equiv) in THF (5 mL) was added dropwise within $10 \mathrm{~min}$. The reaction mixture was stirred for $1 \mathrm{~h}$ at $-78{ }^{\circ} \mathrm{C}$ before iodoalkane 12 (480 mg, $1.5 \mathrm{mmol}, 1.5$ equiv) in dry THF $(3 \mathrm{~mL})$ was introduced at this temperature. The mixture was stirred for 1 hour at $-78^{\circ} \mathrm{C}$. Then the mixture was treated with saturated $\mathrm{NH}_{4} \mathrm{Cl}$ solution $(20 \mathrm{~mL})$ and warmed to room temperature. The mixture was extracted with ethyl acetate $(3 \times 20 \mathrm{~mL})$. The combined organic extracts were washed with saturated brine, dried over $\mathrm{Na}_{2} \mathrm{SO}_{4}$, filtered, and concentrated, and the residue purified by flash 
chromatography (ethyl acetate/petroleum ether $=1 / 40, \mathrm{v} / \mathrm{v})$ to afford the corresponding ent-8 $(364 \mathrm{mg}$, $50 \%$ yield) as a yellow oil and starting material SI-3 (215 mg, $0.4 \mathrm{mmol})$.

$\mathrm{R}_{\mathrm{f}}=0.5$ (ethyl acetate/petroleum ether $=1 / 8, \mathrm{v} / \mathrm{v}$ )

${ }^{1}$ H NMR (500 MHz, CDCl $)_{3}$ both diastereomers $\delta 7.83(\mathrm{~d}, J=7.1 \mathrm{~Hz}, 2 \mathrm{H}), 7.44(\mathrm{~d}, J=7.5 \mathrm{~Hz}, 2 \mathrm{H})$, 7.42-7.35 (m, 3H), $7.32(\mathrm{~d}, J=8.6 \mathrm{~Hz}, 2 \mathrm{H}), 7.26-7.20(\mathrm{~m}, 1 \mathrm{H}), 6.28(\mathrm{~s}, 1 \mathrm{H}), 5.72(\mathrm{~d}, J=5.4 \mathrm{~Hz}, 1 \mathrm{H})$, $5.26(\mathrm{t}, J=7.2 \mathrm{~Hz}, 1 \mathrm{H}), 3.95(\mathrm{~s}, 3 \mathrm{H}), 3.86(\mathrm{~s}, 3 \mathrm{H}), 3.55(\mathrm{~s}, 2 \mathrm{H}), 3.40-3.17(\mathrm{~m}, 2 \mathrm{H}), 2.42-2.14(\mathrm{~m}, 2 \mathrm{H})$, $1.95-1.88(\mathrm{~m}, 1 \mathrm{H}), 1.84(\mathrm{~s}, 3 \mathrm{H}), 1.82-1.76(\mathrm{~m}, 1 \mathrm{H}), 1.71-1.60(\mathrm{~m}, 1 \mathrm{H}), 1.56(\mathrm{~s}, 3 \mathrm{H}), 1.54-1.47(\mathrm{~m}$, $1 \mathrm{H}), 1.45-1.35(\mathrm{~m}, 1 \mathrm{H}), 1.31-1.21(\mathrm{~m}, 1 \mathrm{H}), 1.05-0.91(\mathrm{~m}, 1 \mathrm{H}), 0.84(\mathrm{~s}, 9 \mathrm{H}),-0.03(\mathrm{~d}, J=2.1 \mathrm{~Hz}, 6 \mathrm{H})$.

${ }^{13}$ C NMR (126 MHz, $\mathbf{C D C l}_{3}$ ) major diastereomers $\delta$ 152.4, 152.3, 150.8, 147.3, 136.3, 134.2, 131.7, $131.7,130.9,128.8,128.7,128.5,127.2$, 126.5, 125.2, 124.7, 105.4, 97.6, 91.6, 63.1, 61.6, 56.5, 55.8, 49.7, 44.3, 40.3, 38.3, 34.1, 31.8, 26.0, 24.5, 23.7, 22.4, 18.3, 15.4, -5.3, -5.2.

HRMS (ESI) m/z: $[\mathrm{M}+\mathrm{H}]^{+}$Calcd for $\mathrm{C}_{43} \mathrm{H}_{58} \mathrm{NO}_{5} \mathrm{SSi} 728.3799$; Found 726.3637.

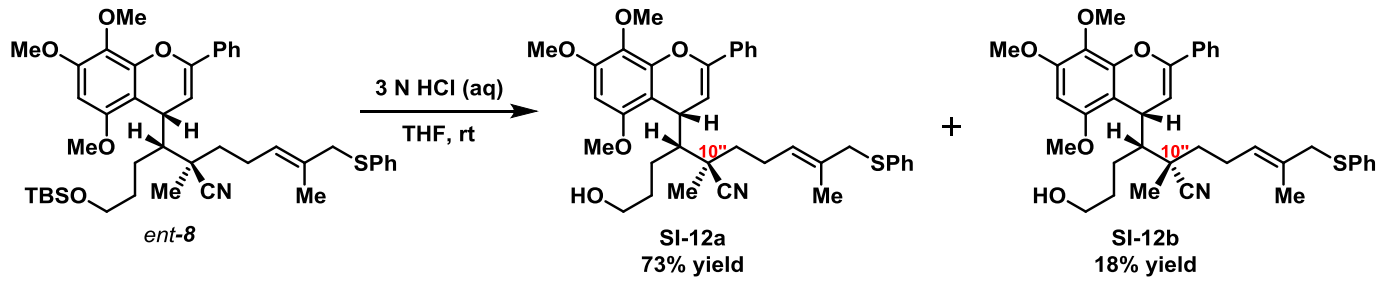

To a solution of ent-8 (1.84 g, $2.55 \mathrm{mmol}, 1.0$ equiv) in THF $(20 \mathrm{~mL})$ at room temperature was added $3 \mathrm{~N}$ aqueous hydrogen chloride $(9 \mathrm{~mL})$. The resulting mixture was stirred at room temperature until ent-5 were consumed completely as judged by TLC, the reaction mixture was diluted by water (10 $\mathrm{mL})$, and extracted with ethyl acetate $(3 \times 20 \mathrm{~mL})$. The combined organic extracts were washed with saturated brine, dried over $\mathrm{Na}_{2} \mathrm{SO}_{4}$, filtered, and concentrated, and the residue purified by flash chromatography (ethyl acetate/petroleum ether $=1 / 3$, v/v) to afford the corresponding SI-12a $(1.15 \mathrm{~g}$, 73\%yield) and SI-12b (110 mg, $18 \%$ yield).

SI-12a: $R_{\mathrm{f}}=0.5$ (ethyl acetate/petroleum ether $\left.=1 / 3, \mathrm{v} / \mathrm{v}\right) .[\alpha]_{\mathrm{D}}^{25.0}=+85.20\left(c 0.1025, \mathrm{CHCl}_{3}\right)$.

${ }^{1} \mathbf{H}$ NMR $\left(\mathbf{5 0 0} \mathbf{M H z}_{\mathbf{C}} \mathbf{C D C l}_{3}\right) \delta 7.77(\mathrm{~d}, J=7.2 \mathrm{~Hz}, 2 \mathrm{H}), 7.39(\mathrm{~d}, J=7.5 \mathrm{~Hz}, 2 \mathrm{H}), 7.36-7.30(\mathrm{~m}, 3 \mathrm{H})$, $7.28-7.22(\mathrm{~m}, 2 \mathrm{H}), 7.17(\mathrm{~d}, J=7.6 \mathrm{~Hz}, 1 \mathrm{H}), 6.23(\mathrm{~s}, 1 \mathrm{H}), 5.65(\mathrm{~d}, J=5.6 \mathrm{~Hz}, 1 \mathrm{H}), 5.21(\mathrm{t}, J=7.2 \mathrm{~Hz}$, $1 \mathrm{H}), 4.12(\mathrm{~d}, J=2.9 \mathrm{~Hz}, 1 \mathrm{H}), 3.89(\mathrm{~s}, 6 \mathrm{H}), 3.80(\mathrm{~s}, 3 \mathrm{H}), 3.49(\mathrm{~s}, 2 \mathrm{H}), 3.28(\mathrm{~d}, J=4.1 \mathrm{~Hz}, 2 \mathrm{H}), 2.21(\mathrm{dd}$ $J=34.2,6.4 \mathrm{~Hz}, 2 \mathrm{H}), 1.92-1.85(\mathrm{~m}, 1 \mathrm{H}), 1.79(\mathrm{~s}, 3 \mathrm{H}), 1.76-1.62(\mathrm{~m}, 2 \mathrm{H}), 1.50(\mathrm{~s}, 3 \mathrm{H}), 1.47-1.42(\mathrm{~m}$, $1 \mathrm{H}), 1.41-1.34(\mathrm{~m}, 1 \mathrm{H}), 1.27-1.17(\mathrm{~m}, 1 \mathrm{H}), 0.97-0.90(\mathrm{~m}, 1 \mathrm{H})$.

${ }^{13}$ C NMR (126 MHz, $\left.\mathbf{C D C l}_{3}\right) \delta 152.3,152.2,150.9,147.3,136.2,134.0,131.8,131.6,130.8,128.8$, $128.7,128.5,127.1,126.5,125.1,124.7,105.3,97.7,91.7,62.7,61.5,56.5,55.8,49.4,44.2,40.4,38.2$, $33.8,31.6,24.5,23.6,22.4,15.4$.

HRMS (ESI) m/z: [M + H] $]^{+}$Calcd for $\mathrm{C}_{37} \mathrm{H}_{44} \mathrm{NO}_{5} \mathrm{~S}$ 614.2935; Found 614.2938.

SI-12b: $R_{\mathrm{f}}=0.35$ (ethyl acetate/petroleum ether $\left.=1 / 3, \mathrm{v} / \mathrm{v}\right) .[\alpha]_{\mathrm{D}}{ }^{25.0}=+88.5\left(c 0.265, \mathrm{CHCl}_{3}\right)$.

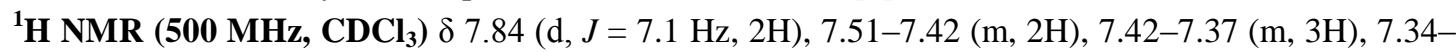
$7.28(\mathrm{~m}, 2 \mathrm{H}), 7.25-7.18(\mathrm{~m}, 1 \mathrm{H}), 6.29(\mathrm{~s}, 1 \mathrm{H}), 5.71(\mathrm{~d}, J=5.5 \mathrm{~Hz}, 1 \mathrm{H}), 5.26(\mathrm{t}, J=6.9 \mathrm{~Hz}, 1 \mathrm{H}), 4.22-$ $4.08(\mathrm{~m}, 1 \mathrm{H}), 3.95(\mathrm{~s}, 6 \mathrm{H}), 3.86(\mathrm{~s}, 3 \mathrm{H}), 3.55(\mathrm{~s}, 2 \mathrm{H}), 3.40-3.25(\mathrm{~m}, 2 \mathrm{H}), 2.42-2.26(\mathrm{~m}, 1 \mathrm{H}), 2.25-2.15$ (m, 1H), 2.02-1.89 (m, 1H), $1.86(\mathrm{~s}, 3 \mathrm{H}), 1.82-1.75(\mathrm{~m}, 1 \mathrm{H}), 1.74-1.59(\mathrm{~m}, 2 \mathrm{H}), 1.56-1.46(\mathrm{~m}, 1 \mathrm{H})$, $1.44(\mathrm{~s}, 3 \mathrm{H}), 1.29-1.23(\mathrm{~m}, 1 \mathrm{H}), 1.04-0.91(\mathrm{~m}, 1 \mathrm{H})$.

${ }^{13}$ C NMR (126 MHz, $\left.\mathbf{C D C l}_{3}\right) \delta 152.3,152.3,150.7,147.2,135.9,134.0,131.7,131.6,131.2,128.7$, $128.7,128.5,127.2$, 126.6, 125.1, 124.9, 105.2, 97.5, 91.5, 62.6, 61.5, 56.5, 55.6, 49.0, 44.4, 40.0, 37.3, 33.9, 31.9, 24.2, 23.6, 23.0, 15.2.

HRMS (ESI) m/z: [M+ H] $]^{+}$Calcd for $\mathrm{C}_{37} \mathrm{H}_{44} \mathrm{NO}_{5} \mathrm{~S}$ 614.2935; Found 614.2938. 

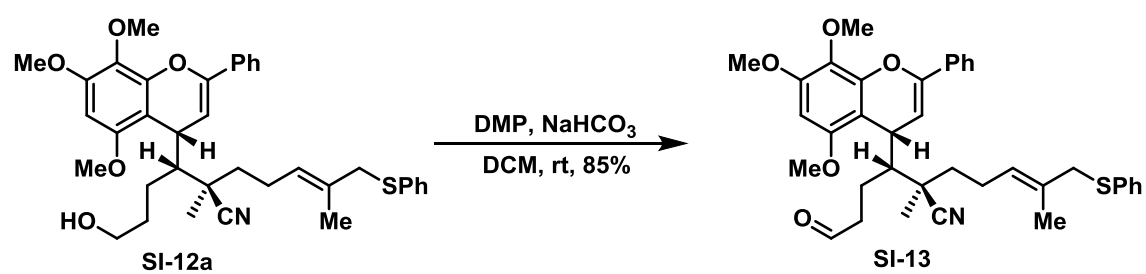

Dess-Martin reagent (377 mg, $0.89 \mathrm{~mol}, 1.5$ equiv) was added in one portion to a stirred solution of alcohol SI-12a (364 mg, 0.59 mol, 1.0 equiv) and $\mathrm{NaHCO}_{3}$ (299 mg, $3.6 \mathrm{mmol}, 6.0$ equiv) in DCM (10 $\mathrm{mL}$ ) at $0{ }^{\circ} \mathrm{C}$. The mixture was allowed to warm to room temperature and stirred for $5 \mathrm{~h}$ before it was treated with water $(20 \mathrm{~mL})$ and stirred for additional $15 \mathrm{~min}$. The organic phase was separated and the aqueous phase extracted with DCM $(3 \times 20 \mathrm{~mL})$. The combined organic layers were washed with saturated $\mathrm{NaCl}$ solution, dried over $\mathrm{Na}_{2} \mathrm{SO}_{4}$, filtered, and concentrated in vacuo. The residue was purified by flash chromatography (ethyl acetate/petroleum ether $=1 / 5, \mathrm{v} / \mathrm{v}$ ). to afford the corresponding SI-13 (307 mg, 85\% yield) as a colorless oil. $[\alpha]_{\mathrm{D}}^{25.0}=+56.52\left(c 0.1075, \mathrm{CHCl}_{3}\right) . \mathrm{R}_{\mathrm{f}}=0.60$ (ethyl acetate/petroleum ether $=1 / 4, \mathrm{v} / \mathrm{v}$ )

${ }^{1} \mathbf{H}$ NMR (500 MHz, $\left.\mathbf{C D C l}_{3}\right) \delta 9.27(\mathrm{~s}, 1 \mathrm{H}), 7.77(\mathrm{~d}, J=7.1 \mathrm{~Hz}, 2 \mathrm{H}), 7.47-7.38(\mathrm{~m}, 2 \mathrm{H}), 7.37-7.30(\mathrm{~m}$ $3 \mathrm{H}), 7.28-7.22(\mathrm{~m}, 2 \mathrm{H}), 7.20-7.12(\mathrm{~m}, 1 \mathrm{H}), 6.24(\mathrm{~s}, 1 \mathrm{H}), 5.66(\mathrm{~d}, J=5.5 \mathrm{~Hz}, 1 \mathrm{H}), 5.30-5.11(\mathrm{~m}, 1 \mathrm{H})$, 4.16-4.10 (m, 1H), 3.90 (s, 3H), 3.89 (s, 3H), 3.80 (s, 3H), 3.50 (s, 2H), 2.28-2.14 (m, 2H), 2.08-2.00 (m, 1H), 1.99-1.90 (m, 3H), 1.79 (s, 3H), 1.76-1.68 (m, 1H), 1.52 (s, 3H), 1.44-1.37 (m, 1H).

${ }^{13}$ C NMR (126 MHz, $\left.\mathbf{C D C l}_{3}\right) \delta$ 201.6, 152.5, 152.2, 150.9, 147.1, 136.2, 133.8, 131.9, 131.6, 130.9, $128.9,128.7,128.6,126.9,126.5,125.0,124.3,104.8,97.2,91.7,61.6,56.5,55.7,48.2$, 44.2, 43.9, $40.1,38.1,31.4,23.5,22.4,20.6,15.4$.

HRMS (ESI) m/z: [M + H] $]^{+}$Calcd for $\mathrm{C}_{37} \mathrm{H}_{42} \mathrm{NO}_{5} \mathrm{~S}$ 612.2778; Found 612.2784.
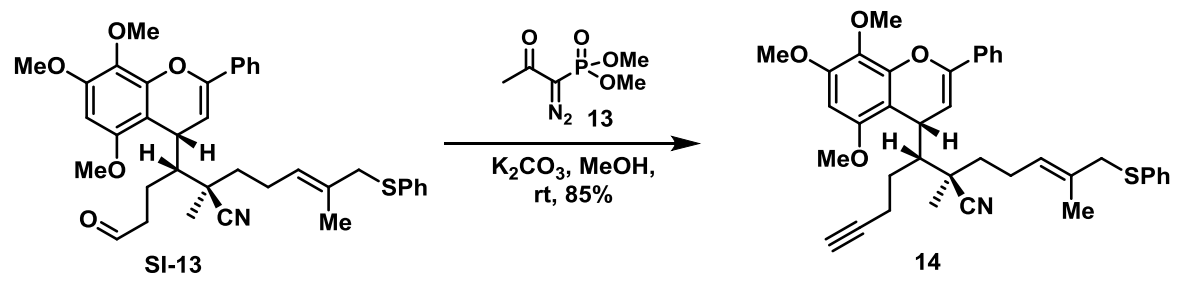

To a stirred solution of potassium carbonate (138 mg, $1.0 \mathrm{mmol}, 2.0$ equiv) in $\mathrm{MeOH}(1 \mathrm{~mL})$ at $0{ }^{\circ} \mathrm{C}$ was added a solution of Ohira-Bestmann reagent $(192 \mathrm{mg}, 1.0 \mathrm{mmol}, 2.0$ equiv) in $\mathrm{MeOH}(1 \mathrm{~mL})$ dropwise. After $10 \mathrm{~min}$, a solution of aldehyde $\mathbf{S I - 1 3}$ (308 mg, $0.5 \mathrm{mmol}, 1.0$ equiv) in $\mathrm{MeOH}(1 \mathrm{~mL})$ was added dropwise. The reaction mixture was stirred at room temperature for $4 \mathrm{~h}$. The reaction mixture was quenched by water at $0{ }^{\circ} \mathrm{C}$. The aqueous layer was extracted with ethyl acetate $(3 \times 4 \mathrm{~mL})$. The combined organic layers were washed with brine, dried over $\mathrm{Na}_{2} \mathrm{SO}_{4}$, and concentrated in vacuo. The crude residue was purified by flash column chromatography (ethyl acetate/petroleum ether $=1 / 10$, $\mathrm{v} / \mathrm{v})$. The desired acetylene $14(258 \mathrm{mg}, 85 \%$ yield $)$ as a colorless oil. $[\alpha]_{\mathrm{D}}{ }^{25.0}=+50.92\left(c 0.04, \mathrm{CHCl}_{3}\right)$. $\mathrm{R}_{\mathrm{f}}=0.52$ (ethyl acetate/petroleum ether $=1 / 8, \mathrm{v} / \mathrm{v}$ )

${ }^{1} \mathbf{H}$ NMR (500 MHz, $\left.\mathbf{C D C l}_{3}\right) \delta 7.84(\mathrm{~d}, J=7.2 \mathrm{~Hz}, 2 \mathrm{H}), 7.50-7.44(\mathrm{~m}, 2 \mathrm{H}), 7.42-7.37$ (m, 3H), 7.33$7.29(\mathrm{~m}, 2 \mathrm{H}), 7.27-7.19(\mathrm{~m}, 1 \mathrm{H}), 6.29(\mathrm{~s}, 1 \mathrm{H}), 5.72(\mathrm{~d}, J=5.4 \mathrm{~Hz}, 1 \mathrm{H}), 5.26(\mathrm{t}, J=7.2 \mathrm{~Hz}, 1 \mathrm{H}), 4.27-$ $4.17(\mathrm{~m}, 1 \mathrm{H}), 3.96(\mathrm{~s}, 3 \mathrm{H}), 3.95(\mathrm{~s}, 3 \mathrm{H}), 3.55(\mathrm{~s}, 2 \mathrm{H}), 2.41-2.19(\mathrm{~m}, 2 \mathrm{H}), 2.20-2.10(\mathrm{~m}, 1 \mathrm{H}), 2.08-1.91$ $(\mathrm{m}, 1 \mathrm{H}), 1.85(\mathrm{~s}, 3 \mathrm{H}), 1.83-1.77(\mathrm{~m}, 3 \mathrm{H}), 1.70-1.62(\mathrm{~m}, 1 \mathrm{H}), 1.58(\mathrm{~s}, 3 \mathrm{H}), 1.55-1.51(\mathrm{~m}, 1 \mathrm{H}), 1.48-$ $1.41(\mathrm{~m}, 1 \mathrm{H})$.

${ }^{13}$ C NMR (126 MHz, $\left.\mathbf{C D C l}_{3}\right) \delta 152.4,152.3,150.9,147.1,136.2,134.0,131.7,131.7,130.8,128.8$, $128.7,128.5,127.1,126.5,125.1,124.4,104.8,97.2,91.8,83.8,68.6,61.6,56.5,55.7,47.5,44.2$, 39.6, $38.1,31.5,27.1,23.5,22.3,18.8,15.4$.

HRMS (ESI) m/z: [M + H] ${ }^{+}$Calcd for $\mathrm{C}_{38} \mathrm{H}_{42} \mathrm{NO}_{4} \mathrm{~S}$ 608.2829; Found 608.2830. 

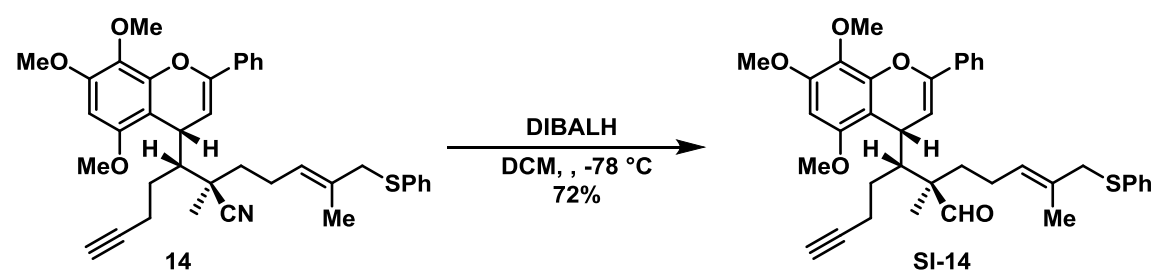

To a solution of acetylene 14 (258 $\mathrm{mg}, 0.42 \mathrm{mmol}, 1.0$ equiv) in anhydrous $\mathrm{CH}_{2} \mathrm{Cl}_{2}(5 \mathrm{~mL})$ was added slowly DIBAL-H $\left(0.71 \mathrm{~mL}, 1.5 \mathrm{M}\right.$ solution in Toluene, $1.06 \mathrm{mmol}, 2.5$ equiv) at $-78^{\circ} \mathrm{C}$. The reaction was stirred at $-78{ }^{\circ} \mathrm{C}$ for $4 \mathrm{~h}$ and the TLC analysis indicated the complete conversion from the starting material to the product. The reaction was quenched with water $(20 \mathrm{~mL})$ at $-78^{\circ} \mathrm{C}$ carefully, and the resulting mixture warm up to room temperature and poured into an ice-cold $5 \%$ aqueous solution of tartaric acid. The mixture was extracted with DCM and washed with saturated $\mathrm{NaHCO}_{3}$ and brine, the combined organic layers were dried $\left(\mathrm{Na}_{2} \mathrm{SO}_{4}\right)$ and evaporated. The residue was purified by flash column chromatography (ethyl acetate/petroleum ether $=1 / 10, \mathrm{v} / \mathrm{v}$ ) to afford the corresponding alcohol SI-14 $\left(188 \mathrm{mg}, 72 \%\right.$ yield) as a colorless oil. $[\alpha]_{\mathrm{D}}{ }^{25.0}=+57.78\left(c 0.1055, \mathrm{CHCl}_{3}\right) . \mathrm{R}_{\mathrm{f}}=0.61$ (ethyl acetate/petroleum ether $=1 / 8, \mathrm{v} / \mathrm{v}$ )

${ }^{1} \mathbf{H}$ NMR $\left(\mathbf{5 0 0} \mathbf{~ M H z}, \mathbf{C D C l}_{3}\right) \delta 9.52(\mathrm{~s}, 1 \mathrm{H}), 7.69(\mathrm{~d}, J=7.6 \mathrm{~Hz}, 2 \mathrm{H}), 7.43-7.37(\mathrm{~m}, 2 \mathrm{H}), 7.37-7.31(\mathrm{~m}$ $3 \mathrm{H}), 7.27-7.21(\mathrm{~m}, 3 \mathrm{H}), 6.25(\mathrm{~s}, 1 \mathrm{H}), 5.38(\mathrm{~d}, J=5.7 \mathrm{~Hz}, 1 \mathrm{H}), 5.19(\mathrm{t}, J=7.3 \mathrm{~Hz}, 1 \mathrm{H}), 4.07-4.00(\mathrm{~m}$, $1 \mathrm{H}), 3.90$ (s, 3H), 3.89 (s, 3H), 3.83 (s, 3H), 3.49 (s, 2H), 2.44-2.27 (m, 1H), 2.02-1.86 (m, 2H), 1.74 (s, 3H), 1.73-1.62 (m, 4H), 1.58-1.40 (m, 3H), 1.19 (s, 3H).

${ }^{13}$ C NMR (126 MHz, $\mathbf{C D C l}_{3}$ ) $\delta$ 206.5, 152.3, 152.2, 150.7, 147.2, 136.3, 133.9, 131.7, 131.1, 130.8, $128.7,128.7,128.5,128.2,126.4,124.7,106.0,99.0,91.9,84.2,68.4,61.5,56.5,55.7,53.0,46.2,44.3$, $34.7,30.9,26.1,22.8,18.7,16.9,15.3$.

HRMS (ESI) m/z: $[\mathrm{M}+\mathrm{H}]^{+}$Calcd for $\mathrm{C}_{38} \mathrm{H}_{43} \mathrm{O}_{5} \mathrm{~S}$ 611.2826; Found 611.2826.
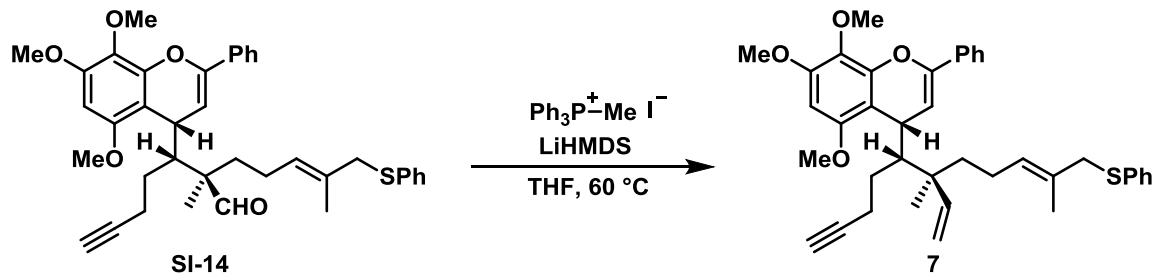

To a stirred solution of methyltriphenylphosphonium iodide ( $300 \mathrm{mg}, 0.75 \mathrm{mmol}, 2.5$ equiv) in 2 $\mathrm{mL}$ of THF at $0{ }^{\circ} \mathrm{C}$ was slowly added LiHMDS ( $0.75 \mathrm{~mL}, 1.0 \mathrm{M}$ in THF, $0.75 \mathrm{mmol}, 2.5$ equiv). After stirring for $30 \mathrm{~min}$, the solution was warmed to $\mathrm{rt}$ and was stirred for an additional $1 \mathrm{~h}$. The mixture was cooled to $0{ }^{\circ} \mathrm{C}$ and a solution of aldehyde SI-14 (188 mg, $0.3 \mathrm{mmol}, 1.0$ equiv) in THF ( $1 \mathrm{~mL}$ ) was added dropwise and the mixture was allowed to warm to $60{ }^{\circ} \mathrm{C}$ and was stirred for $2 \mathrm{~h}$. The reaction was quenched with $\mathrm{H}_{2} \mathrm{O}$ and extracted with ethyl acetate $(3 \times 4 \mathrm{~mL})$. The combined organic layer was dried over $\mathrm{Na}_{2} \mathrm{SO}_{4}$ and the solvent was removed under reduced pressure. The crude residue was purified by flash silica gel column chromatography (ethyl acetate/petroleum ether $=1 / 10, \mathrm{v} / \mathrm{v}$ ) to provide alkene 7 $\left(157 \mathrm{mg}, 86 \%\right.$ yield) as a yellow oil. $[\alpha]_{\mathrm{D}}^{25.0}=+35.3\left(c 0.333, \mathrm{CHCl}_{3}\right) . \mathrm{R}_{\mathrm{f}}=0.61$ (ethyl acetate/petroleum ether $=1 / 9, \mathrm{v} / \mathrm{v})$

${ }^{1}$ H NMR $\left(\mathbf{5 0 0 ~ M H z}, \mathbf{C D C l}_{\mathbf{3}}\right): \delta$ 7.72-7.68 $(\mathrm{m}, 2 \mathrm{H}), 7.40(\mathrm{t}, J=7.6 \mathrm{~Hz}, 2 \mathrm{H}), 7.36-7.31(\mathrm{~m}, 3 \mathrm{H})$, 7.28-7.23 (m, 2H), $7.16(\mathrm{t}, J=7.4 \mathrm{~Hz}, 1 \mathrm{H}), 6.24(\mathrm{~s}, 1 \mathrm{H}), 5.88-5.80(\mathrm{~m}, 1 \mathrm{H}), 5.60(\mathrm{~d}, J=5.7 \mathrm{~Hz}, 1 \mathrm{H})$, $5.23(\mathrm{t}, J=7.2 \mathrm{~Hz}, 1 \mathrm{H}), 5.15-5.10(\mathrm{~m}, 1 \mathrm{H}), 5.02-4.96(\mathrm{~m}, 1 \mathrm{H}), 4.12-4.08(\mathrm{~m}, 1 \mathrm{H}), 3.90(\mathrm{~s}, 3 \mathrm{H}), 3.89(\mathrm{~s}$, $3 \mathrm{H}), 3.82(\mathrm{~s}, 3 \mathrm{H}), 3.50(\mathrm{~s}, 2 \mathrm{H}), 1.98-1.88(\mathrm{~m}, 3 \mathrm{H}), 1.75(\mathrm{~s}, 3 \mathrm{H}), 1.71(\mathrm{t}, J=2.2 \mathrm{~Hz}, 1 \mathrm{H}), 1.68-1.52(\mathrm{~m}$, $4 \mathrm{H}), 1.45-1.33(\mathrm{~m}, 2 \mathrm{H}), 1.20(\mathrm{~s}, 3 \mathrm{H})$. 
${ }^{13} \mathbf{C}$ NMR (126 MHz, $\left.\mathbf{C D C l}_{3}\right): \delta 152.5,152.0,149.7,147.4,146.4,136.6,134.5,131.8,130.8,130.1$, 129.7, 128.8, 128.5, 128.4, 126.4, 124.7, 112.6, 107.3, 100.6, 92.1, 85.0, 67.9, 61.6, 56.7, 55.8, 51.0, 44.5, 43.9, 39.6, 31.4, 26.0, 23.2, 20.6, 19.5, 15.3.

HRMS (ESI) m/z: [M + H] ${ }^{+}$Calcd for $\mathrm{C}_{39} \mathrm{H}_{45} \mathrm{O}_{4} \mathrm{~S}$ 609.3033; Found 609.3023.
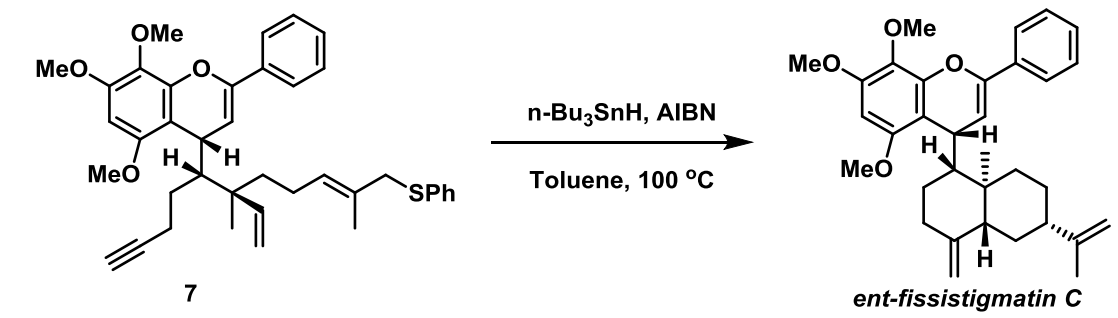
$(10 \%)$
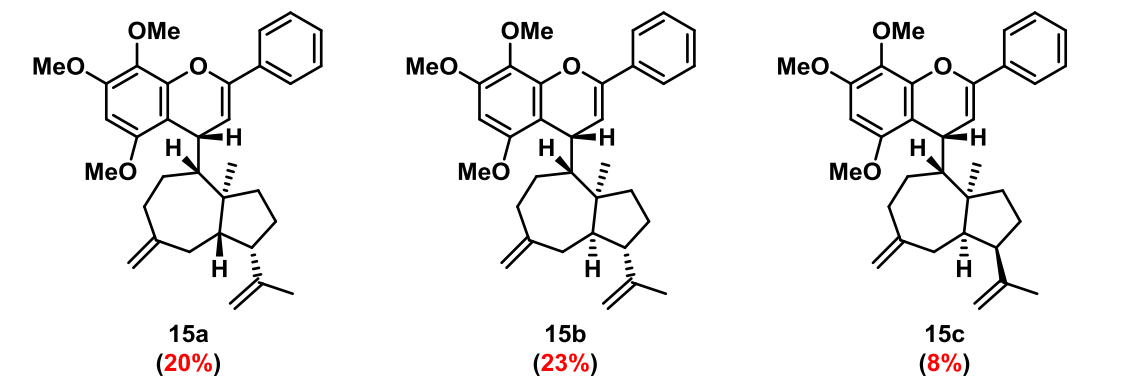

To a stirred solution of 7 (61 mg, $0.1 \mathrm{mmol}, 1.0$ equiv) in $9 \mathrm{~mL}$ of Toluene at $80{ }^{\circ} \mathrm{C}$ was slowly added a solution of AIBN ( $49 \mathrm{mg}, 0.3 \mathrm{mmol}, 3.0$ equiv) and $n-\mathrm{Bu}_{3} \mathrm{SnH}(80 \mu \mathrm{L}, 0.3 \mathrm{mmol}, 3.0$ equiv) in $1 \mathrm{~mL}$ of Toluene within $30 \mathrm{~min}$. The resulting mixture was stirred at same temperature until 7 were consumed completely as judged by TLC, then cooled to room temperature and $\mathrm{TsOH} \bullet \mathrm{H}_{2} \mathrm{O}(285 \mathrm{mg}$, $1.5 \mathrm{mmol}, 5.0$ equiv) was added one a portion, and the resulting mixture stirred at room temperature for $30 \mathrm{~min}$ before it was treated with water and the organic phase was separated and the aqueous phase extracted with ethyl acetate $(3 \times 10 \mathrm{~mL})$. The combined organic layers were washed with saturated $\mathrm{NaCl}$ solution, dried over $\mathrm{Na}_{2} \mathrm{SO}_{4}$, filtered, and concentrated in vacuo. The residue was purified by flash chromatography (ethyl acetate/petroleum ether $=1 / 20, \mathrm{v} / \mathrm{v}$ ). to afford the corresponding products as a mixture of diastereoisomers $(12 \mathrm{mg}, 58 \%$ yield). The mixture was separated via LC semi-prep column chromatography (Exsil Mono C18 $5 \mu \mathrm{m}, \mathrm{MeOH} / \mathrm{H}_{2} \mathrm{O}=95: 5,2 \mathrm{~mL} / \mathrm{min}$ ) to afford ent-fissistigmatin $\boldsymbol{C}(6.5 \mathrm{mg}, 10 \%)$ as a white solid, $15 \boldsymbol{a}(13.1 \mathrm{mg}, 20 \%)$ as a white solid, $15 \boldsymbol{b}(15.1 \mathrm{mg}$, $23 \%)$ as a off-white solid and $\mathbf{1 5 c}(5.2 \mathrm{mg}, 8 \%)$ as a white solid. $\mathrm{R}_{\mathrm{f}}=0.66$ (ethyl acetate/petroleum ether $=1 / 9, \mathrm{v} / \mathrm{v}$ )

${ }^{1}$ H NMR (500 MHz, acetone- $\left.d_{6}\right) \delta 7.76(\mathrm{~d}, J=7.3 \mathrm{~Hz}, 1 \mathrm{H}), 7.42(\mathrm{t}, J=7.5 \mathrm{~Hz}, 2 \mathrm{H}), 7.35(\mathrm{t}, J=7.3$ $\mathrm{Hz}, 1 \mathrm{H}), 6.51(\mathrm{~s}, 1 \mathrm{H}), 5.67(\mathrm{~d}, J=6.0 \mathrm{~Hz}, 1 \mathrm{H}), 4.77(\mathrm{~s}, 1 \mathrm{H}), 4.71(\mathrm{~s}, 1 \mathrm{H}), 4.63(\mathrm{~s}, 1 \mathrm{H}), 4.43(\mathrm{~s}, 1 \mathrm{H})$, 4.22-4.11 (m, 1H), $3.90(\mathrm{~s}, 3 \mathrm{H}), 3.89(\mathrm{~s}, 3 \mathrm{H}), 3.82(\mathrm{~s}, 3 \mathrm{H}), 2.33-2.27(\mathrm{~m}, 1 \mathrm{H}), 2.26-2.20(\mathrm{~m}, 1 \mathrm{H})$, 2.03-1.99 (m, 1H), $1.90(\mathrm{~m}, 1 \mathrm{H}), 1.86(\mathrm{~m}, 1 \mathrm{H}), 1.78(\mathrm{~s}, 3 \mathrm{H}), 1.74-1.66(\mathrm{~m}, 2 \mathrm{H}), 1.65-1.57(\mathrm{~m}, 2 \mathrm{H})$, $1.56-1.51(\mathrm{~m}, 1 \mathrm{H}), 1.50-1.42(\mathrm{~m}, 3 \mathrm{H}), 0.94(\mathrm{~s}, 3 \mathrm{H})$.

${ }^{13}$ C NMR (126 MHz, acetone- $\boldsymbol{d}_{\boldsymbol{6}}$ ) $\delta$ 152.9, 152.8, 151.5, 151.4, 150.5, 148.2, 135.3, 132.3, 129.3, 129.2, 125.2, 108.8, 108.7, 105.7, 101.8, 93.5, 61.3, 57.2, 56.7, 56.2, 51.8, 46.3, 40.6, 39.5, 37.3, 30.6, $30.1,27.9,25.8,21.2,15.1$.

HRMS (ESI) m/z: [M + H] $]^{+}$Calcd for $\mathrm{C}_{33} \mathrm{H}_{41} \mathrm{O}_{4}$ 501.2999; Found 501.2993.

Table S2. Specific Rotation Data for ent-fissistigmatin C, Compound 15a, 15b and 15c

\begin{tabular}{lccccc}
\hline Entry & ent-fissistigmatin C & Fissistigmatin C $\left(\right.$ lit. $\left.^{\mathbf{3}}\right)$ & $\mathbf{1 5 a}$ & $\mathbf{1 5 b}$ & $\mathbf{1 5 c}$ \\
\hline$c \mathrm{~g} / 100 \mathrm{~mL}, \mathrm{MeOH}$ & 0.060 & 0.050 & 0.038 & 0.098 & 0.045 \\
{$[\alpha]_{\mathrm{D}}^{25}$} & +148.2 & -162.2 & +111.3 & +127.4 & +101.8 \\
\hline
\end{tabular}




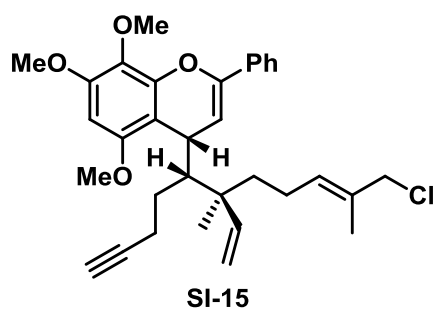

$\mathrm{R}_{\mathrm{f}}=0.40$ (ethyl acetate/petroleum ether $\left.=1 / 10, \mathrm{v} / \mathrm{v}\right)$, yellow oil $(7.5 \mathrm{mg}, 70 \%$ yield).

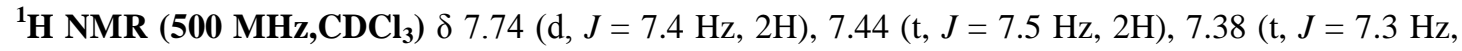
$1 \mathrm{H}), 6.28(\mathrm{~s}, 1 \mathrm{H}), 5.98-5.84(\mathrm{~m}, 1 \mathrm{H}), 5.66(\mathrm{~d}, J=5.8 \mathrm{~Hz}, 1 \mathrm{H}), 5.60(\mathrm{t}, J=6.9 \mathrm{~Hz}, 1 \mathrm{H}), 5.19(\mathrm{~d}, J=$ $10.9 \mathrm{~Hz}, 1 \mathrm{H}), 5.06(\mathrm{~d}, J=16.6 \mathrm{~Hz}, 1 \mathrm{H}), 4.20-4.14(\mathrm{~m}, 1 \mathrm{H}), 4.07(\mathrm{~s}, 2 \mathrm{H}), 3.94(\mathrm{~s}, 6 \mathrm{H}), 3.88(\mathrm{~s}, 3 \mathrm{H})$, $2.08(\mathrm{~d}, J=10.4 \mathrm{~Hz}, 2 \mathrm{H}), 1.99(\mathrm{~d}, J=6.8 \mathrm{~Hz}, 1 \mathrm{H}), 1.80(\mathrm{~s}, 3 \mathrm{H}), 1.77$ (s, 1H), 1.75-1.59 (m, 4H), 1.59$1.50(\mathrm{~m}, 2 \mathrm{H}), 1.28(\mathrm{~s}, 3 \mathrm{H})$.

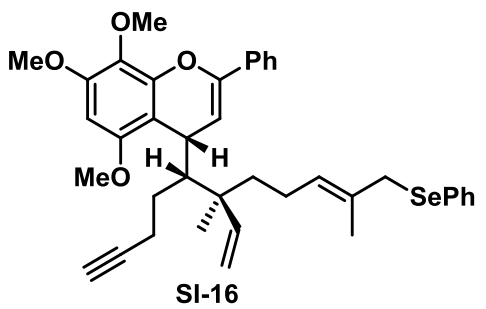

$\mathrm{R}_{\mathrm{f}}=0.50$ (ethyl acetate/petroleum ether $\left.=1 / 4, \mathrm{v} / \mathrm{v}\right)$, yellow oil $(10.4 \mathrm{mg}, 80 \%$ yield).

${ }^{1}$ H NMR (500 MHz, $\left.\mathbf{C D C l}_{3}\right) \delta 7.69(\mathrm{~d}, J=7.3 \mathrm{~Hz}, 2 \mathrm{H}), 7.52-7.45(\mathrm{~m}, 2 \mathrm{H}), 7.40(\mathrm{t}, J=7.5 \mathrm{~Hz}, 2 \mathrm{H})$, 7.36-7.30 (m, 1H), 7.25-7.20 (m, 3H), $6.23(\mathrm{~s}, 1 \mathrm{H}), 5.89-5.77(\mathrm{~m}, 1 \mathrm{H}), 5.59$ (d, J = 5.8 Hz, 1H), 5.16$5.07(\mathrm{~m}, 2 \mathrm{H}), 4.98(\mathrm{~d}, J=17.5 \mathrm{~Hz}, 1 \mathrm{H}), 4.16-4.02(\mathrm{~m}, 1 \mathrm{H}), 3.90(\mathrm{~s}, 6 \mathrm{H}), 3.82(\mathrm{~s}, 3 \mathrm{H}), 3.51(\mathrm{~s}, 2 \mathrm{H})$, 1.95-1.85 (m, 3H), 1.75 (s, 3H), $1.71(\mathrm{~s}, 1 \mathrm{H}), 1.68-1.58(\mathrm{~m}, 2 \mathrm{H}), 1.54-1.48(\mathrm{~m}, 1 \mathrm{H}), 1.42-1.23(\mathrm{~m}$, $3 \mathrm{H}), 1.19$ (s, 1H).

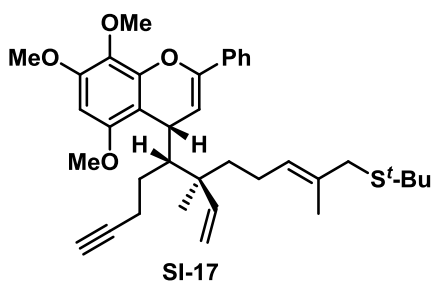

$\mathrm{R}_{\mathrm{f}}=0.50$ (ethyl acetate/petroleum ether $\left.=1 / 8, \mathrm{v} / \mathrm{v}\right)$, yellow oil $(6.0 \mathrm{mg}, 76 \%$ yield).

${ }^{1} \mathbf{H}$ NMR (500 MHz, CDCl $) \delta 7.69(\mathrm{~d}, J=7.4 \mathrm{~Hz}, 2 \mathrm{H}), 7.39(\mathrm{t}, J=7.5 \mathrm{~Hz}, 2 \mathrm{H}), 7.35-7.30(\mathrm{~m}, 1 \mathrm{H})$, $6.23(\mathrm{~s}, 1 \mathrm{H}), 5.98-5.78(\mathrm{~m}, 1 \mathrm{H}), 5.61(\mathrm{~d}, J=5.7 \mathrm{~Hz}, 1 \mathrm{H}), 5.38(\mathrm{t}, J=7.5 \mathrm{~Hz}, 1 \mathrm{H}), 5.13(\mathrm{~d}, J=11.0 \mathrm{~Hz}$, 1H), $5.00(\mathrm{~d}, J=17.5 \mathrm{~Hz}, 1 \mathrm{H}), 4.17-4.08(\mathrm{~m}, 1 \mathrm{H}), 3.89$ (s, 6H), 3.83 (s, 3H), 3.19 (s, 2H), 2.04-1.96 $(\mathrm{m}, 2 \mathrm{H}), 1.96-1.89(\mathrm{~m}, 1 \mathrm{H}), 1.74(\mathrm{~s}, 3 \mathrm{H}), 1.73-1.70(\mathrm{~m}, 1 \mathrm{H}), 1.70-1.64(\mathrm{~m}, 1 \mathrm{H}), 1.62-1.45(\mathrm{~m}, 5 \mathrm{H})$, $1.33(\mathrm{~s}, 9 \mathrm{H}), 1.23(\mathrm{~s}, 3 \mathrm{H})$.

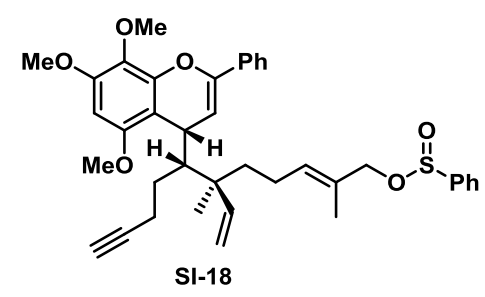

$\mathrm{R}_{\mathrm{f}}=0.50$ (ethyl acetate/petroleum ether $=1 / 2$, v/v), yellow oil $(8.7 \mathrm{mg}, 81 \%$ yield).

${ }^{1}$ H NMR (500 MHz, CDCl $) \delta$ 7.76-7.66 (m, 4H), 7.57-7.51 (m, 3H), $7.39(\mathrm{t}, J=7.5 \mathrm{~Hz}, 2 \mathrm{H}), 7.33(\mathrm{t}$, $J=7.3 \mathrm{~Hz}, 1 \mathrm{H}), 6.22(\mathrm{~s}, 1 \mathrm{H}), 5.91-5.78(\mathrm{~m}, 1 \mathrm{H}), 5.60(\mathrm{~d}, J=5.7 \mathrm{~Hz}, 1 \mathrm{H}), 5.46(\mathrm{t}, J=7.0 \mathrm{~Hz}, 1 \mathrm{H})$, 
$5.13(\mathrm{~d}, J=10.7 \mathrm{~Hz}, 1 \mathrm{H}), 5.00(\mathrm{~d}, J=16.8 \mathrm{~Hz}, 1 \mathrm{H}), 4.43(\mathrm{~d}, J=10.8 \mathrm{~Hz}, 1 \mathrm{H}), 4.13-4.09(\mathrm{~m}, 1 \mathrm{H}), 4.00$ (d, $J=10.9 \mathrm{~Hz}, 1 \mathrm{H}), 3.89$ (s, 6H), $3.82(\mathrm{~s}, 3 \mathrm{H}), 2.04-1.97(\mathrm{~m}, 2 \mathrm{H}), 1.95-1.90(\mathrm{~m}, 1 \mathrm{H}), 1.74-1.70(\mathrm{~m}$, $1 \mathrm{H}), 1.66(\mathrm{~s}, 3 \mathrm{H}), 1.63-1.57$ (m, 3H), 1.51-1.42 (m, 3H), 1.22 (s, 3H).

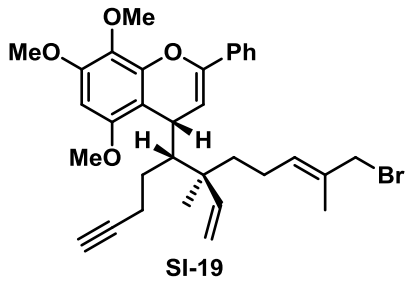

$\mathrm{R}_{\mathrm{f}}=0.47$ (ethyl acetate/petroleum ether $\left.=1 / 10, \mathrm{v} / \mathrm{v}\right)$, yellow oil $(5.1 \mathrm{mg}, 65$ yield).

${ }^{1}$ H NMR (500 MHz, CDCl $) \delta 7.69(\mathrm{~d}, J=7.4 \mathrm{~Hz}, 2 \mathrm{H}), 7.39(\mathrm{t}, J=7.5 \mathrm{~Hz}, 2 \mathrm{H}), 7.33(\mathrm{t}, J=7.4 \mathrm{~Hz}$, $1 \mathrm{H}), 6.23(\mathrm{~s}, 1 \mathrm{H}), 5.91-5.80(\mathrm{~m}, 1 \mathrm{H}), 5.61(\mathrm{~d}, J=5.7 \mathrm{~Hz}, 1 \mathrm{H}), 5.55(\mathrm{t}, J=6.4 \mathrm{~Hz}, 1 \mathrm{H}), 5.15(\mathrm{~d}, J=$ $11.0 \mathrm{~Hz}, 1 \mathrm{H}), 5.02(\mathrm{~d}, J=17.4 \mathrm{~Hz}, 1 \mathrm{H}), 4.16-4.09(\mathrm{~m}, 1 \mathrm{H}), 4.01(\mathrm{~d}, J=21.1 \mathrm{~Hz}, 2 \mathrm{H}), 3.89(\mathrm{~s}, 6 \mathrm{H})$, $3.83(\mathrm{~s}, 3 \mathrm{H}), 2.08-1.99(\mathrm{~m}, 2 \mathrm{H}), 1.96-1.92(\mathrm{~m}, 1 \mathrm{H}), 1.76(\mathrm{~d}, J=9.1 \mathrm{~Hz}, 3 \mathrm{H}), 1.73-1.71(\mathrm{~m}, 1 \mathrm{H}), 1.70$ $1.62(\mathrm{~m}, 2 \mathrm{H}), 1.62-1.56(\mathrm{~m}, 2 \mathrm{H}), 1.52-1.46(\mathrm{~m}, 2 \mathrm{H}), 1.23(\mathrm{~s}, 3 \mathrm{H})$.

Table S3. Tests of radical cascade on various leaving group ${ }^{a}$

\begin{tabular}{cccc}
\hline Entry & Leaving group & ${\text { Yield }(\boldsymbol{\%})^{\mathbf{b}}}$ & ${\text { Ratio of } \mathbf{3} / \mathbf{1 5}^{\mathbf{c}}}^{\mathbf{c}}$ \\
\hline 1 & $\sim \mathrm{SPh}$ & 68 & $1: 7$ \\
2 & $\sim \mathrm{SePh}$ & 25 & $1: 7$ \\
3 & $\sim \mathrm{St}-\mathrm{Bu}$ & 65 & $1: 7$ \\
4 & $\sim \mathrm{Cl}$ & 67 & $1: 7$ \\
5 & $\sim \mathrm{Br}$ & 40 & $1: 7$ \\
6 & $\sim \mathrm{OS}(\mathrm{O}) \mathrm{Ph}$ & 64 & $1: 7$ \\
\hline
\end{tabular}

[a] Conditions: Substrate (0.1 mmol), AIBN (49 mg, $0.3 \mathrm{mmol}, 3.0$ equiv), $n-\mathrm{Bu}_{3} \mathrm{SnH}(80 \mu \mathrm{L}, 0.3 \mathrm{mmol}, 3.0$ equiv), toluene $(\mathrm{c}=0.01 \mathrm{M}), 100{ }^{\circ} \mathrm{C}$. [b] Yields of isolated. [c] Determined by ${ }^{1} \mathrm{H}$ NMR. 
Table S4. ${ }^{1} \mathrm{H} \&{ }^{13} \mathrm{C}$ NMR data of ent-fissistigmatin $C(500 \mathrm{MHz}$, acetone-d $)$

\begin{tabular}{|c|c|c|c|c|}
\hline Position & ${ }^{1} \mathbf{H}$ NMR $\delta^{\text {a }}$ mult. J(Hz) & ${ }^{1}$ H NMR (lit. ${ }^{3}$ ) & ${ }^{13} \mathrm{C}$ NMR & ${ }^{13} \mathrm{C}$ NMR (lit. ${ }^{3}$ ) \\
\hline 2 & -- & -- & 150.5 & 150.4 \\
\hline 3 & $5.67 \mathrm{~d} 6.0$ & $5.68 \mathrm{~d} 6.1$ & 101.8 & 101.7 \\
\hline 4 & $4.16 \mathrm{dd} 6.0 / 1.4$ & $4.16 \mathrm{dd} 6.1 / 1.3$ & 30.6 & 30.5 \\
\hline 5 & -- & -- & 152.9 & 152.9 \\
\hline 5-OMe & $3.90 \mathrm{~s}$ & $3.90 \mathrm{~s}$ & 56.2 & 56.1 \\
\hline 6 & $6.51 \mathrm{~s}$ & $6.51 \mathrm{~s}$ & 93.5 & 93.4 \\
\hline 7 & -- & -- & 152.9 & 152.8 \\
\hline 7-OMe & $3.89 \mathrm{~s}$ & $3.89 \mathrm{~s}$ & 56.7 & 56.6 \\
\hline 8 & -- & -- & 132.3 & 132.2 \\
\hline 8-OMe & $3.82 \mathrm{~s}$ & $3.82 \mathrm{~s}$ & 61.3 & 61.2 \\
\hline 9 & -- & -- & 148.2 & 148.2 \\
\hline 10 & -- & -- & 108.8 & 108.7 \\
\hline $1^{\prime}$ & -- & -- & 135.3 & 135.2 \\
\hline $2^{\prime} / 6^{\prime}$ & $7.76 \mathrm{~d} 7.4$ & $7.76 \mathrm{~d} 7.4$ & 125.2 & 125.2 \\
\hline $3^{\prime} / 5^{\prime}$ & 7.42 t 7.4 & 7.43 t 7.4 & 129.3 & 129.3 \\
\hline $4^{\prime}$ & 7.35 t 7.4 & 7.35 t 7.4 & 129.2 & 129.2 \\
\hline $1^{\prime \prime}$ & 1.67 & 1.68 & 57.2 & 57.1 \\
\hline $2^{\prime \prime}$ & $1.48 / 1.64$ & $1.47 / 1.64^{b}$ & 25.8 & 25.8 \\
\hline $3^{\prime \prime}$ & $1.90 / 2.24$ & $1.90 / 2.24^{b}$ & 37.3 & 37.3 \\
\hline $4^{\prime \prime}$ & -- & -- & 151.4 & 151.4 \\
\hline $5^{\prime \prime}$ & $1.86 \mathrm{brd} 12.2$ & $1.86 \mathrm{brd} 11.5$ & 51.8 & 51.7 \\
\hline $6^{\prime \prime}$ & $1.52 / 1.48$ & $1.52 / 1.49^{b}$ & 30.1 & 30.1 \\
\hline $7^{\prime \prime}$ & 2.02 & 2.02 & 46.3 & 46.3 \\
\hline $8^{\prime \prime}$ & $1.70 / 1.49$ & $1.71 / 1.49^{b}$ & 27.9 & 27.8 \\
\hline $9^{\prime \prime}$ & $1.61 / 2.30$ & $1.61 / 2.29^{b}$ & 39.5 & 39.4 \\
\hline $10^{\prime \prime}$ & -- & -- & 40.6 & 40.5 \\
\hline $11^{\prime \prime}$ & -- & -- & 151.5 & 151.5 \\
\hline $12^{\prime \prime}$ & $4.77 \mathrm{br} \mathrm{s} / 4.71 \mathrm{br} \mathrm{s}$ & $4.77 \mathrm{br} \mathrm{s} / 4.72 \mathrm{br} \mathrm{s}{ }^{\mathrm{c}}$ & 108.8 & 108.8 \\
\hline $13^{\prime \prime}$ & $1.78 \mathrm{~s}$ & $1.78 \mathrm{br} \mathrm{s}$ & 21.2 & 21.1 \\
\hline $14^{\prime \prime}$ & $0.94 \mathrm{~s}$ & $0.94 \mathrm{~s}$ & 15.1 & 15.0 \\
\hline $15^{\prime \prime}$ & $4.43 \mathrm{br} \mathrm{s} / 4.63 \mathrm{br} \mathrm{s}$ & $4.43 \mathrm{br} \mathrm{s} / 4.64 \mathrm{br} \mathrm{s}{ }^{\mathrm{c}}$ & 105.7 & 105.7 \\
\hline
\end{tabular}

${ }^{\text {a }}$ Values in italics are chemical shifts of HSQC correlation peaks.

${ }^{\mathrm{b}} \mathrm{H}-\alpha / \mathrm{H}-\beta$.

${ }^{\mathrm{c}} \mathrm{H}-\mathrm{Z} / \mathrm{H}-\mathrm{E}$. 


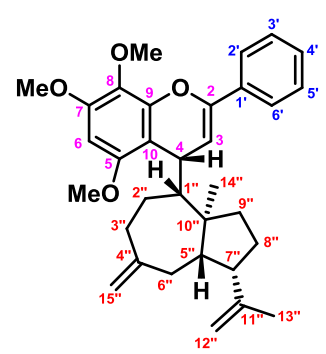

$15 a$

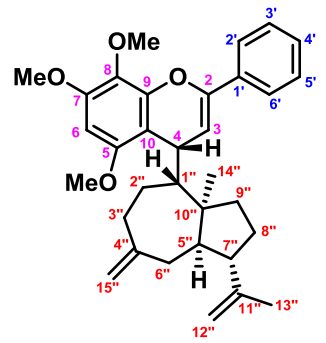

$15 b$

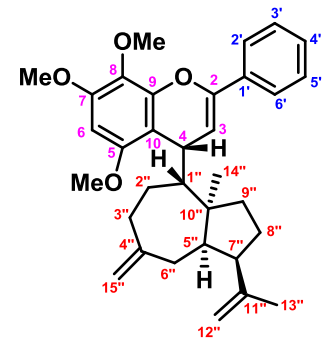

$15 \mathrm{c}$

Table S5. ${ }^{1} \mathrm{H}$ NMR data of compound $15 \mathrm{a}, 15 \mathrm{~b}$ and $15 \mathrm{c}\left(500 \mathrm{MHz}\right.$, acetone-d $\left.{ }_{6}\right)$

\begin{tabular}{|c|c|c|c|}
\hline Position & 15a $\delta^{\mathrm{a}}$ mult. $J(\mathrm{~Hz})$ & 15b $\delta^{\mathrm{a}}$ mult. $J(\mathrm{~Hz})$ & 15c $\delta^{\text {a }}$ mult. $J(\mathrm{~Hz})$ \\
\hline 3 & $5.73 \mathrm{~d} 6.0$ & $5.75 \mathrm{~d} 5.9$ & $5.80 \mathrm{~d} 5.7$ \\
\hline 4 & $4.07 \mathrm{dd} 6.0 / 1.9$ & $3.97 \mathrm{dd} 5.8 / 2.0$ & $3.83 \mathrm{dd} 5.8 / 1.4$ \\
\hline 6 & $6.50 \mathrm{~s}$ & $6.50 \mathrm{~s}$ & $6.52 \mathrm{~s}$ \\
\hline 5-OMe & $3.89 \mathrm{~s}$ & $3.90 \mathrm{~s}$ & $3.92 \mathrm{~s}$ \\
\hline 7-OMe & $3.88 \mathrm{~s}$ & $3.88 \mathrm{~s}$ & $3.88 \mathrm{~s}$ \\
\hline 8-OMe & $3.82 \mathrm{~s}$ & $3.82 \mathrm{~s}$ & $3.81 \mathrm{~s}$ \\
\hline $2 ' / 6$ & $7.76 \mathrm{~d} 7.4$ & $7.77 \mathrm{~d} 7.4$ & $7.77 \mathrm{~d} 7.4$ \\
\hline $3^{\prime} / 5$ & 7.42 t 7.4 & 7.42 t 7.4 & 7.43 t 7.4 \\
\hline $4^{\prime}$ & 7.35 t 7.4 & 7.35 t 7.4 & 7.36 t 7.4 \\
\hline $1 "$ & 1.64 & 2.06 & 2.10 \\
\hline $2 "$ & $1.51 / 1.65^{b}$ & $1.58 / 1.52^{b}$ & $1.36 / 1.61^{b}$ \\
\hline $3 "$ & $2.18 / 1.95^{b}$ & $2.27 / 1.75^{b}$ & $2.31 / 1.62^{b}$ \\
\hline $5 "$ & 2.27 & 1.50 & 1.63 \\
\hline $6 "$ & $2.17 / 2.28^{b}$ & $2.21 / 2.02^{b}$ & $1.81 / 1.84^{b}$ \\
\hline $7 "$ & 2.95 & 2.24 & 2.83 \\
\hline $8 "$ & $1.76 / 1.85^{b}$ & $1.70 / 1.81^{b}$ & $1.75 / 1.80^{b}$ \\
\hline $9 "$ & $1.98 / 1.93^{b}$ & $1.85 / 2.08^{b}$ & $1.73 / 2.49^{b}$ \\
\hline $12 "$ & $4.80 \mathrm{br} \mathrm{s} / 4.91 \mathrm{br} \mathrm{s}^{\mathrm{c}}$ & $4.76 \mathrm{br} \mathrm{s} / 4.73 \mathrm{br} \mathrm{s}{ }^{c}$ & $4.70 \mathrm{br} \mathrm{s} / 4.87 \mathrm{br} \mathrm{s}^{\mathrm{c}}$ \\
\hline $13 "$ & $1.77 \mathrm{~s}$ & $1.75 \mathrm{~s}$ & $1.74 \mathrm{~s}$ \\
\hline $14 "$ & $1.06 \mathrm{~s}$ & $1.23 \mathrm{~s}$ & $1.31 \mathrm{~s}$ \\
\hline $15 "$ & $4.56 \mathrm{br} \mathrm{s} / 4.63 \mathrm{br} \mathrm{s}{ }^{\mathrm{c}}$ & $4.54 \mathrm{br} \mathrm{s} / 4.51 \mathrm{br} \mathrm{s} \mathrm{c}^{\mathrm{c}}$ & $4.42 \mathrm{br} \mathrm{s} / 4.47 \mathrm{br} \mathrm{s}^{\mathrm{c}}$ \\
\hline
\end{tabular}

${ }^{a}$ Values in italics are chemical shifts of HSQC correlation peaks.

${ }^{\mathrm{b}} \mathrm{H}-\alpha / \mathrm{H}-\beta$.

${ }^{\mathrm{c}} \mathrm{H}-\mathrm{Z} / \mathrm{H}-\mathrm{E}$. 


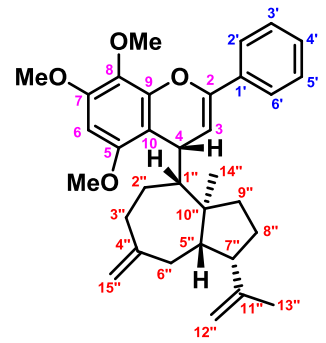

$15 a$

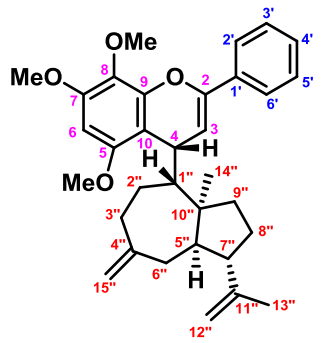

$15 b$

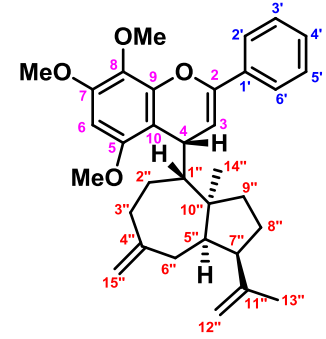

$15 \mathrm{c}$

Table S6. ${ }^{13} \mathrm{C}$ NMR data of compound $15 \mathrm{a}, 15 \mathrm{~b}$ and $15 \mathrm{c}\left(126 \mathrm{MHz}\right.$, acetone-d $\left.{ }_{6}\right)$

\begin{tabular}{|c|c|c|c|}
\hline Position & $15 a$ & $15 \mathrm{~b}$ & $15 \mathrm{c}$ \\
\hline 2 & 150.4 & 150.5 & 150.7 \\
\hline 3 & 101.7 & 101.7 & 102.0 \\
\hline 4 & 34.4 & 34.1 & 34.6 \\
\hline 5 & 153.1 & 153.1 & 153.2 \\
\hline $5-\mathrm{OMe}$ & 56.8 & 56.2 & 56.2 \\
\hline 6 & 93.7 & 93.5 & 93.7 \\
\hline 7 & 153.0 & 152.9 & 153.0 \\
\hline 7-OMe & 56.2 & 56.6 & 56.8 \\
\hline 8 & 132.4 & 132.2 & 132.3 \\
\hline 8-OMe & 61.3 & 61.3 & 61.3 \\
\hline 9 & 148.2 & 148.0 & 147.9 \\
\hline 10 & 108.9 & 108.6 & 108.9 \\
\hline $1^{\prime}$ & 135.3 & 135.3 & 135.5 \\
\hline $2^{\prime} / 6^{\prime}$ & 125.2 & 125.2 & 125.4 \\
\hline $3^{\prime} / 5^{\prime}$ & 129.3 & 129.3 & 129.3 \\
\hline 4' & 129.2 & 129.2 & 129.2 \\
\hline $1 "$ & 59.9 & 51.1 & 56.4 \\
\hline $2 "$ & 30.3 & 26.4 & 26.8 \\
\hline $3 "$ & 35.9 & 38.1 & 40.2 \\
\hline $4 "$ & 152.5 & 150.9 & 153.7 \\
\hline $5 "$ & 48.7 & 56.1 & 54.0 \\
\hline $6 "$ & 34.4 & 39.3 & 33.1 \\
\hline $7 "$ & 50.2 & 56.4 & 50.1 \\
\hline $8 "$ & 29.8 & 30.1 & 27.0 \\
\hline 9" & 41.9 & 40.7 & 37.8 \\
\hline $10 "$ & 50.3 & 48.5 & 50.4 \\
\hline $11 "$ & 147.7 & 148.3 & 147.6 \\
\hline $12 "$ & 112.7 & 110.7 & 111.0 \\
\hline $13 "$ & 24.4 & 19.8 & 23.5 \\
\hline $14 "$ & 18.0 & 26.6 & 28.0 \\
\hline $15 "$ & 110.4 & 110.3 & 109.5 \\
\hline
\end{tabular}




\section{2D NMR data of compound $15 a, 15 b$ and $15 c$}
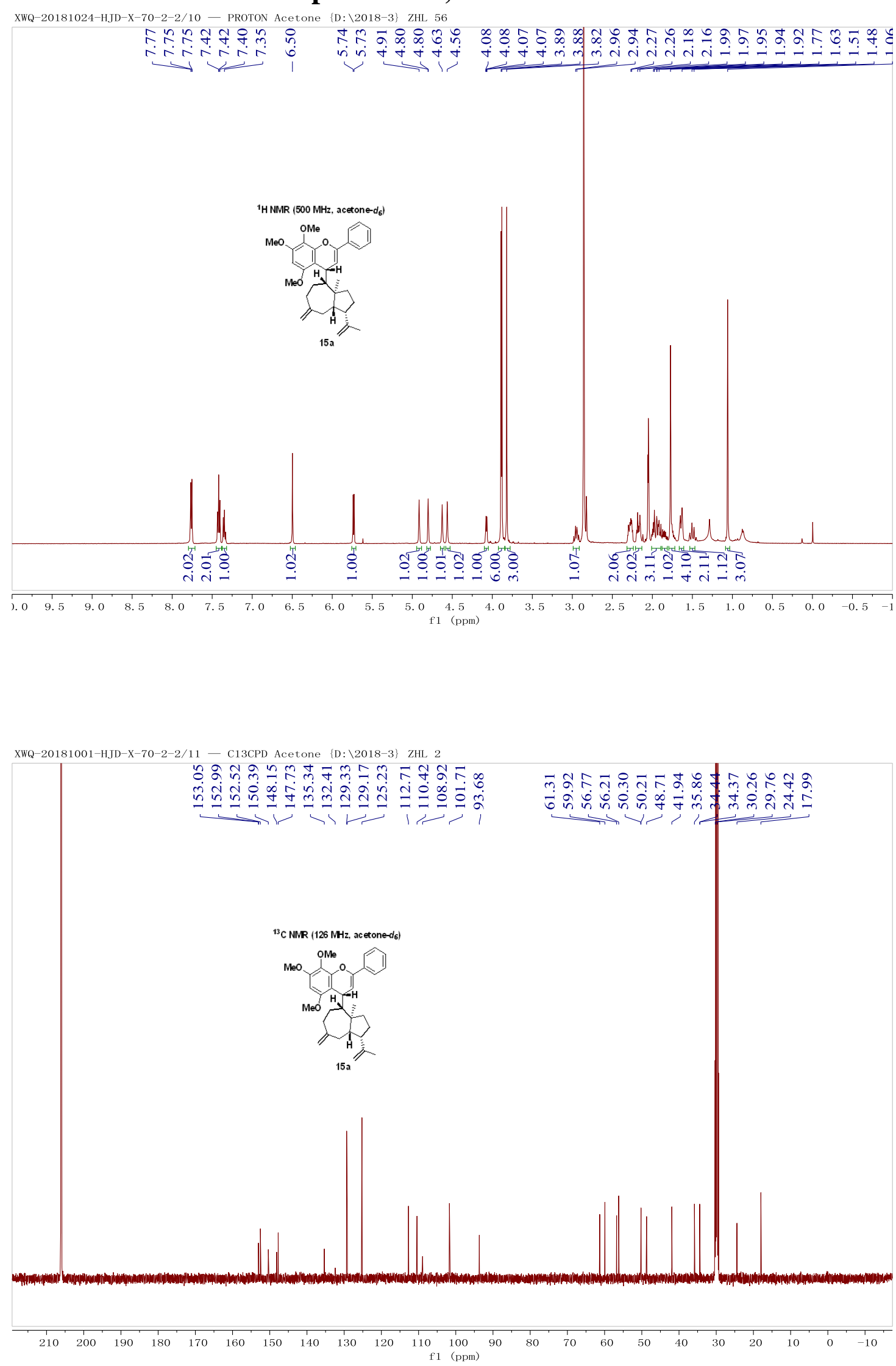


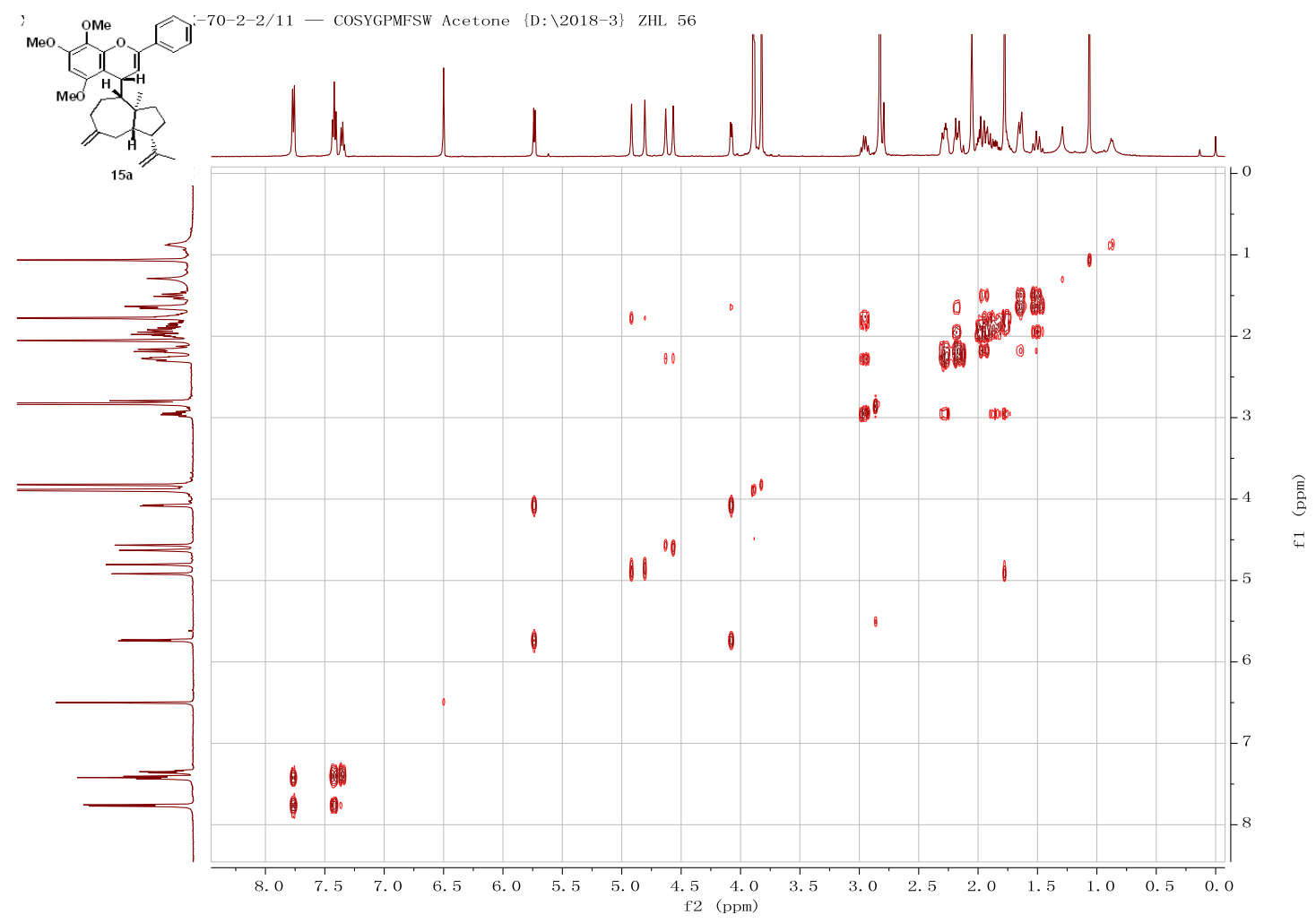

H- ${ }^{1} \mathrm{H}$ COSY NMR (500 MHz, acetone) 


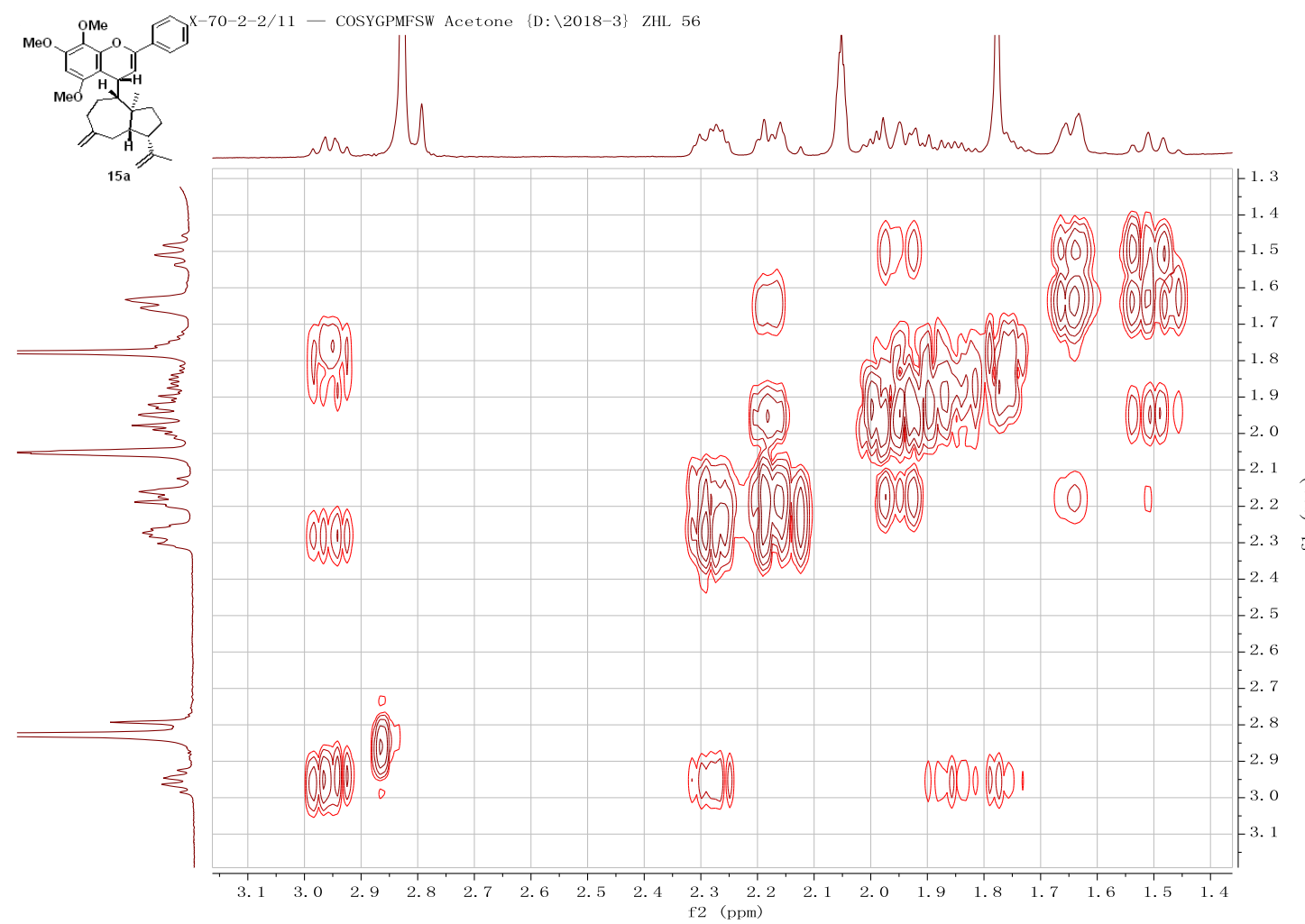

H- ${ }^{1} \mathrm{H}$ COSY NMR (500 MHz, acetone) Expansion 


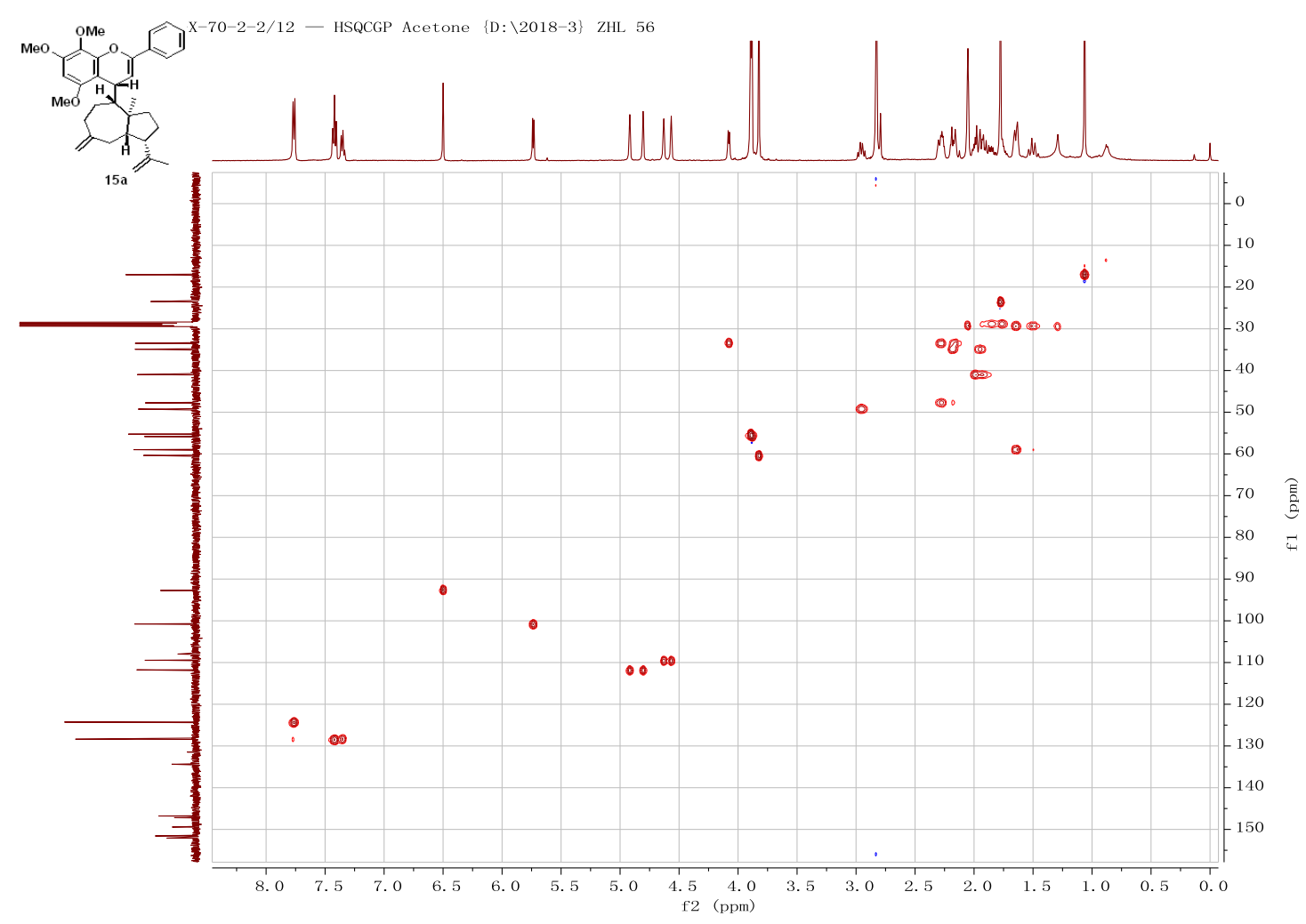

${ }^{1} \mathrm{H}-{ }^{13} \mathrm{C}$ HSQC (500 MHz \& $126 \mathrm{MHz}$ ) of Compound 15a in acetone- $d_{6}$

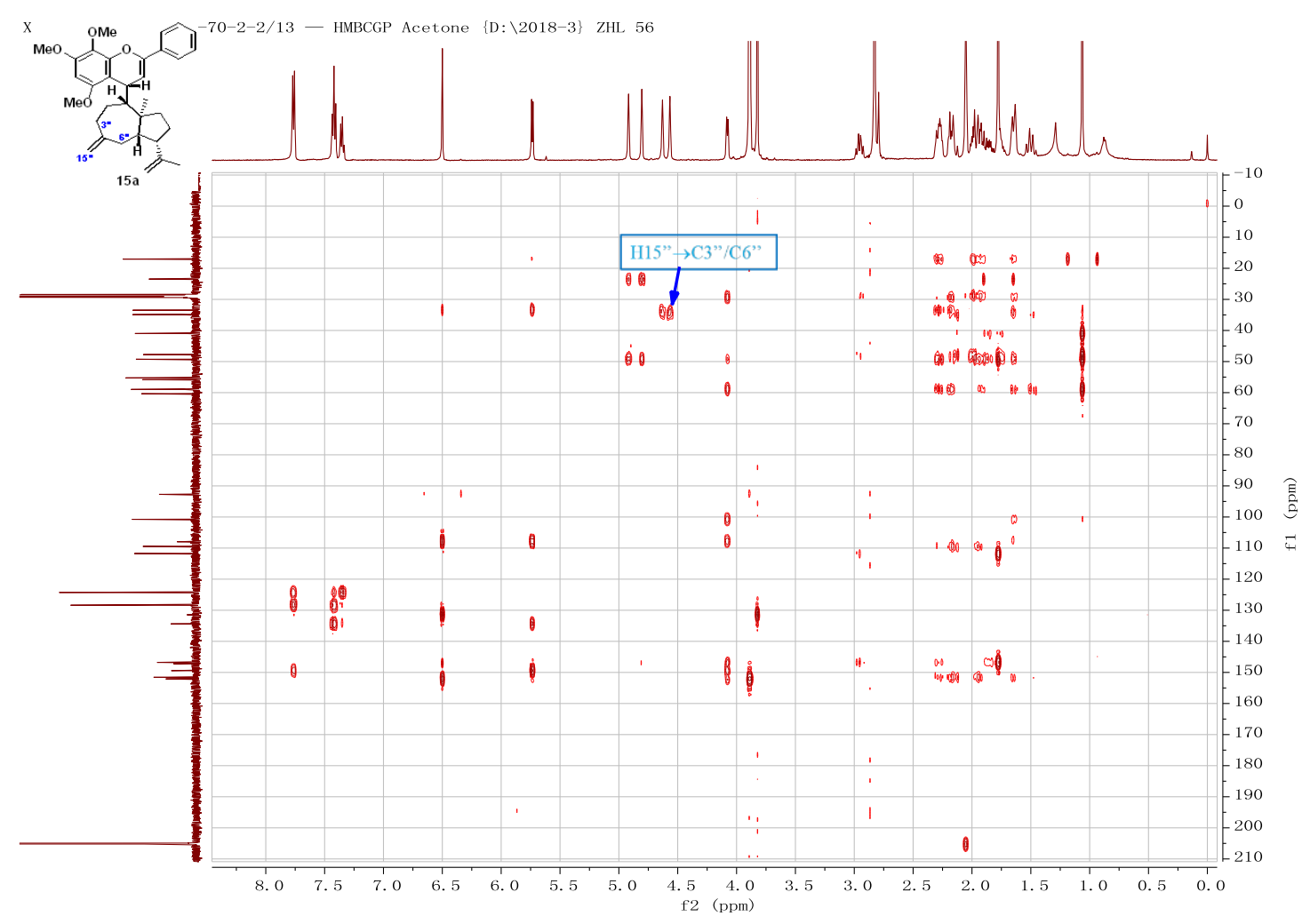

${ }^{1} \mathrm{H}-{ }^{13} \mathrm{C}$ HMBC (500 MHz \& $\left.126 \mathrm{MHz}\right)$ of Compound 15a in acetone- $d_{6}$ 


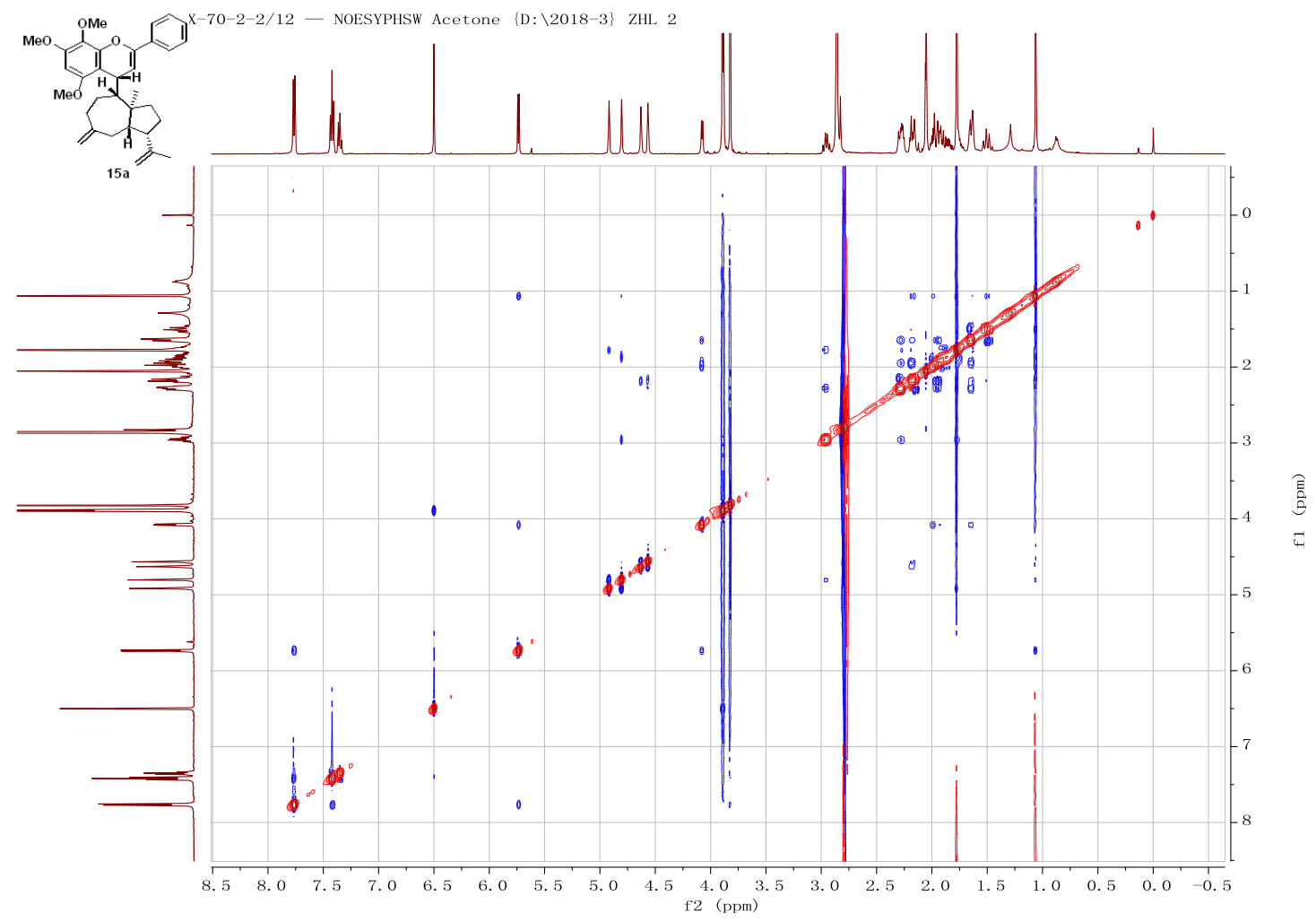

H- ${ }^{1} \mathrm{H}$ NOESY NMR (500 MHz, acetone)

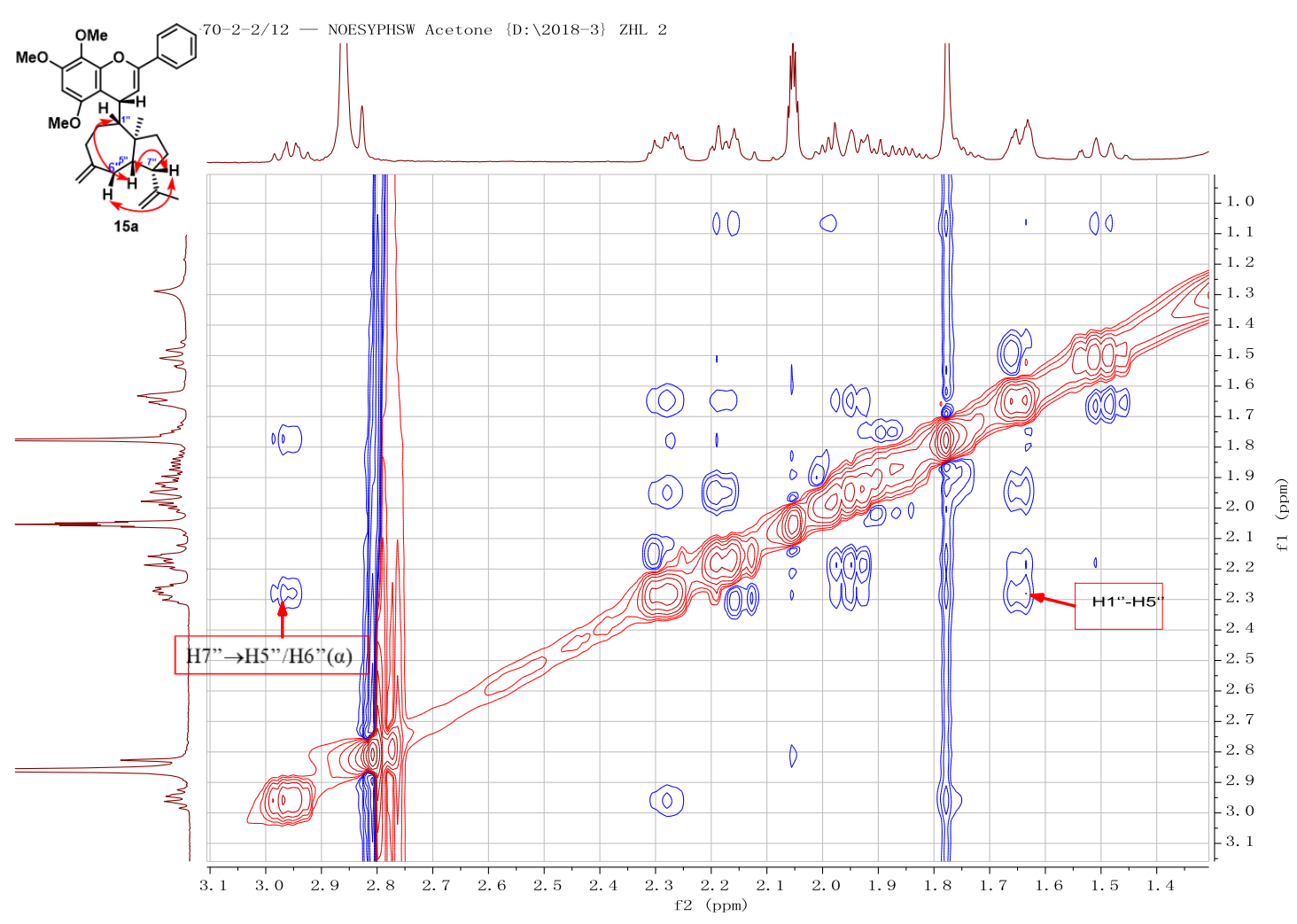

H- ${ }^{1} \mathrm{H}$ NOESY NMR (500 MHz, acetone) Expansion 

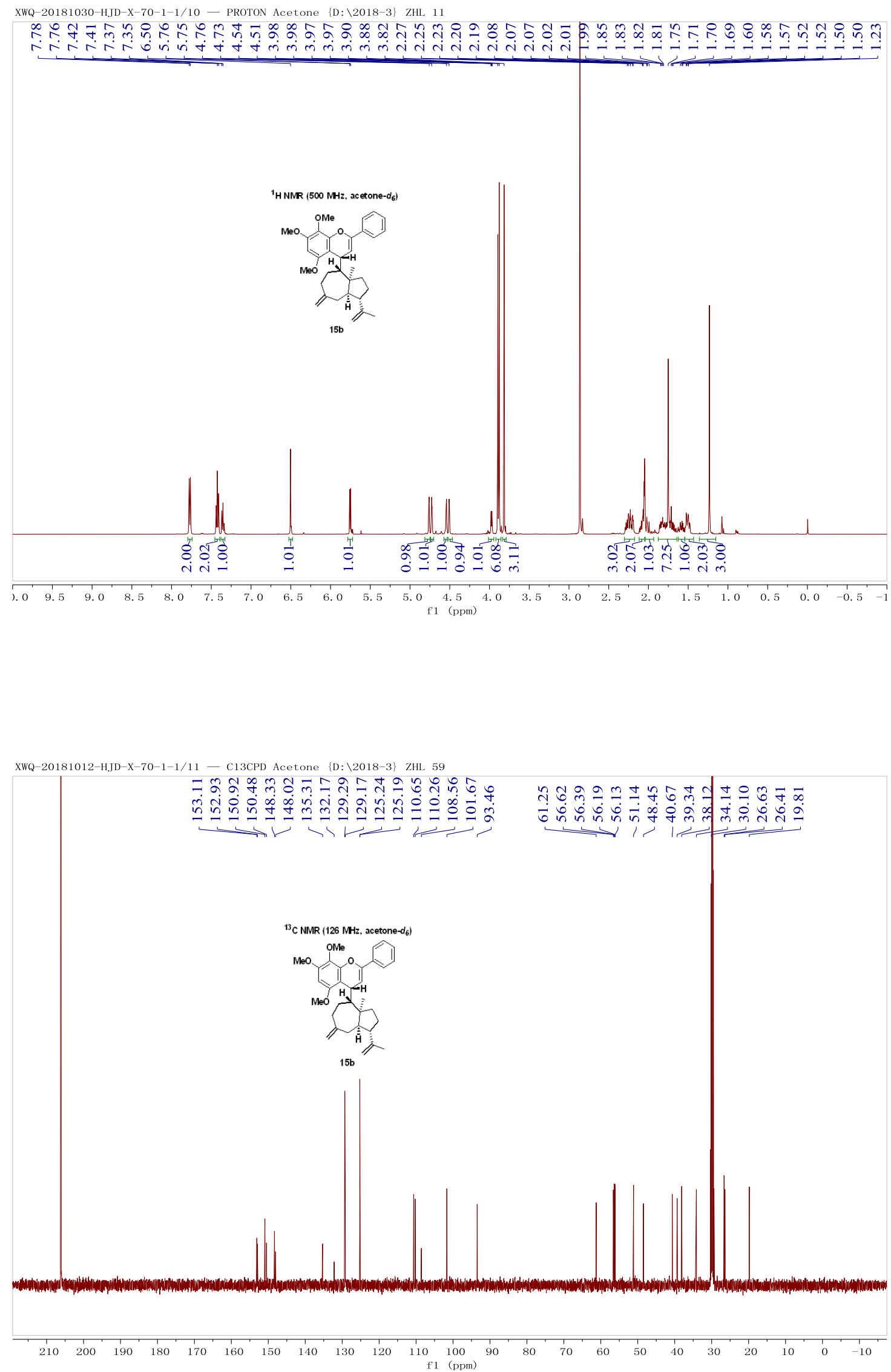


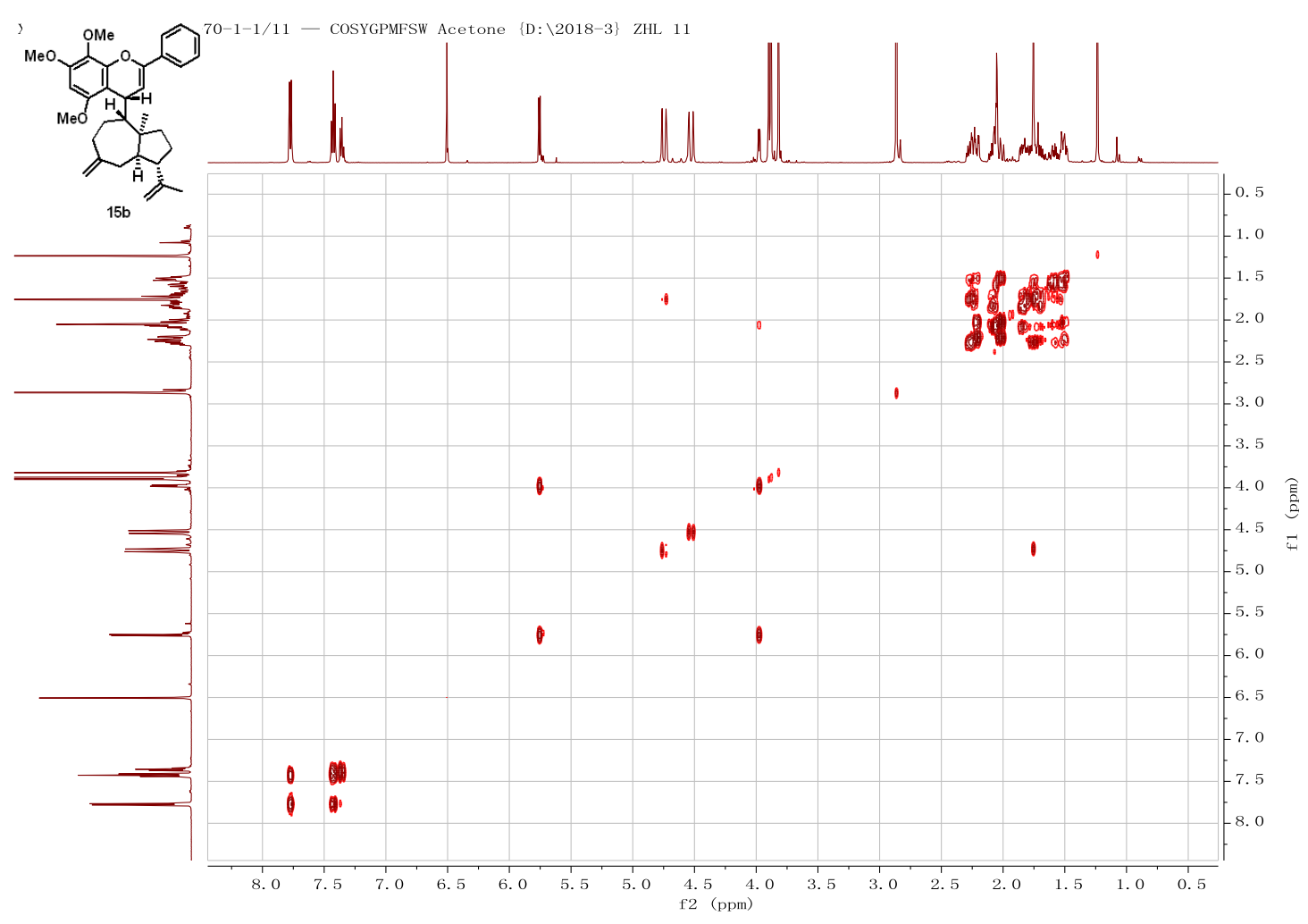

H- ${ }^{1} \mathrm{H}$ COSY NMR (500 MHz, acetone)

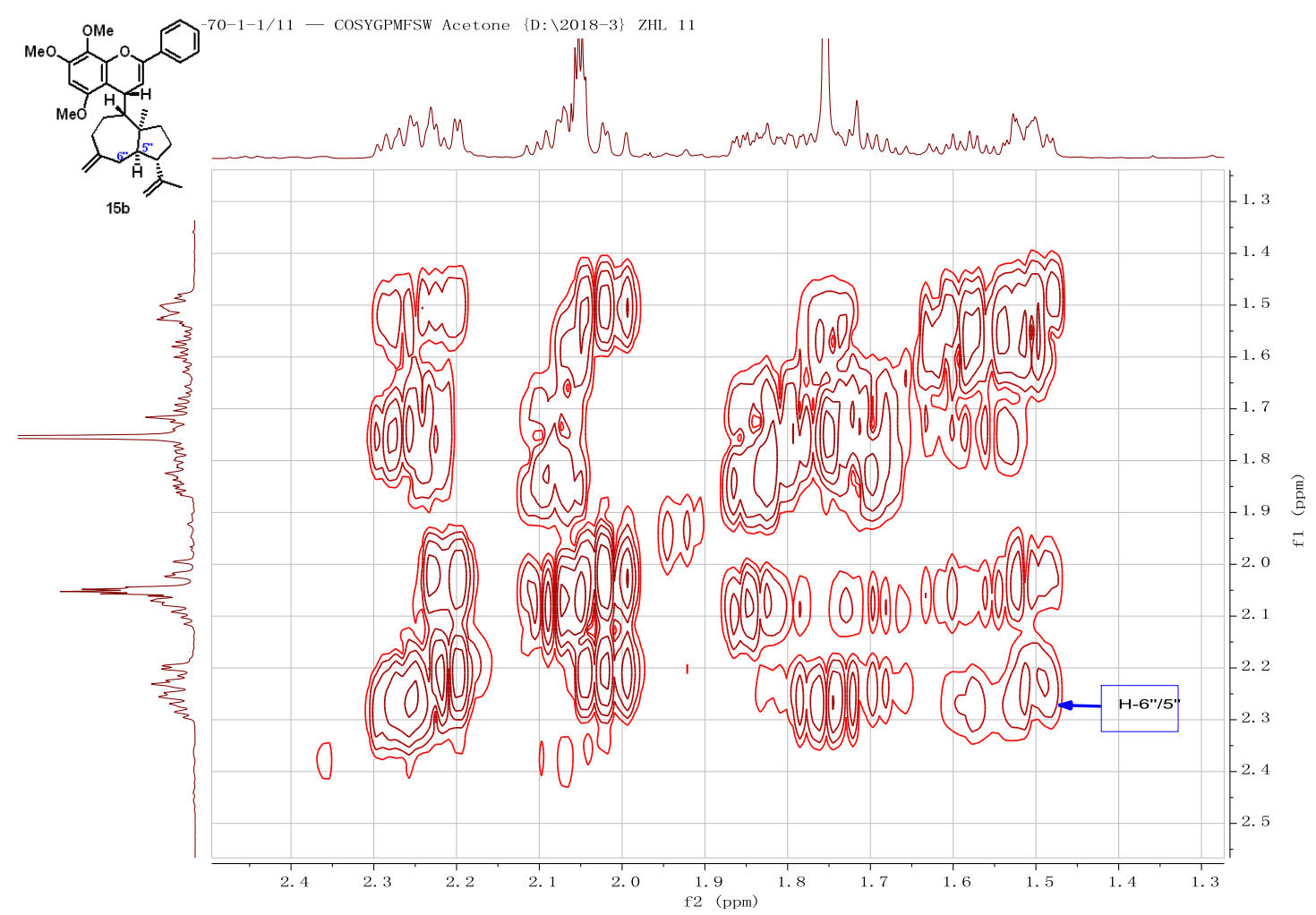

H- ${ }^{1} \mathrm{H}$ COSY NMR (500 MHz, acetone) Expansion 


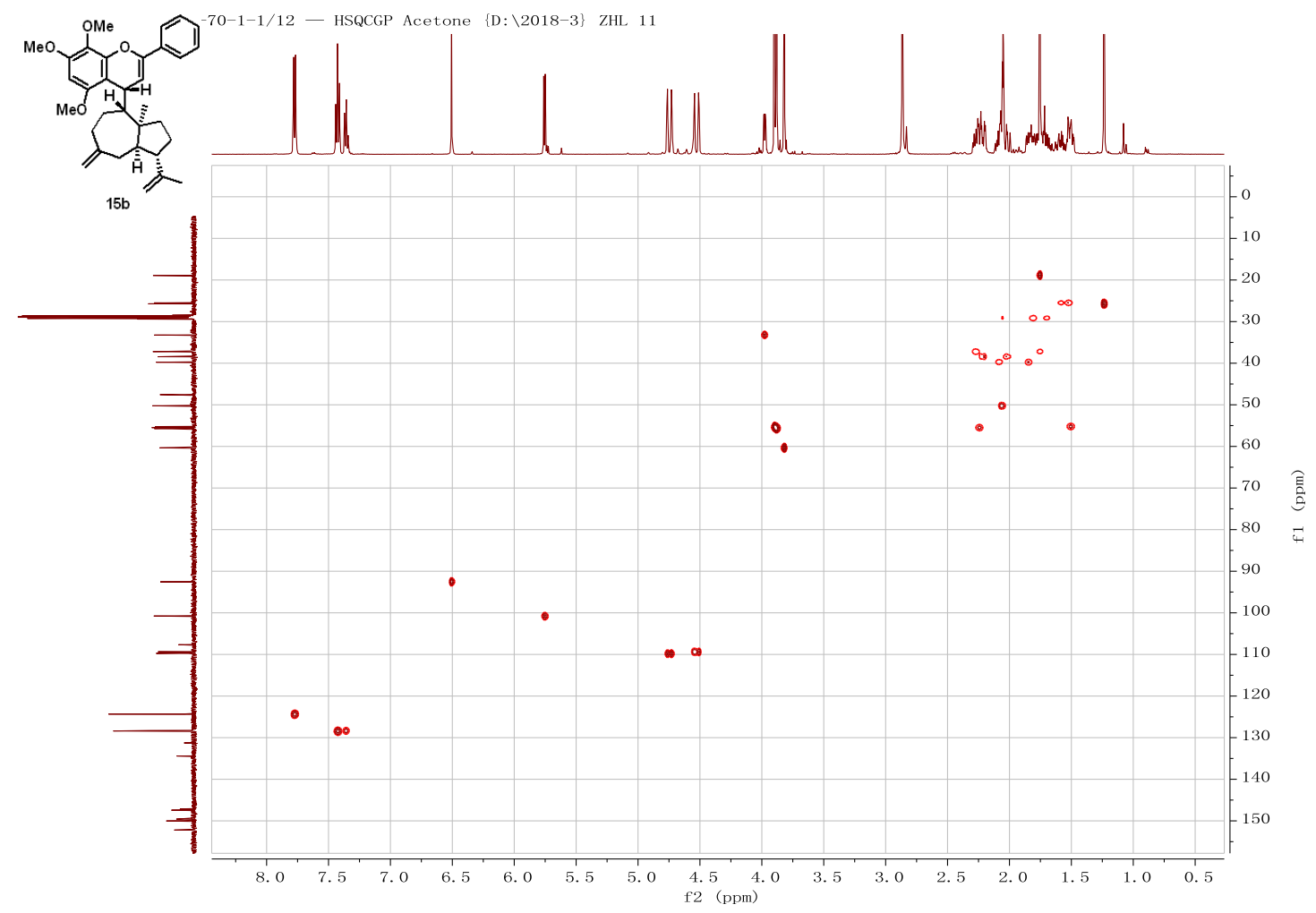

${ }^{1} \mathrm{H}-{ }^{13} \mathrm{C}$ HSQC (500 MHz \& $126 \mathrm{MHz}$ ) of Compound $15 \mathrm{~b}$ in acetone- $d_{6}$ 


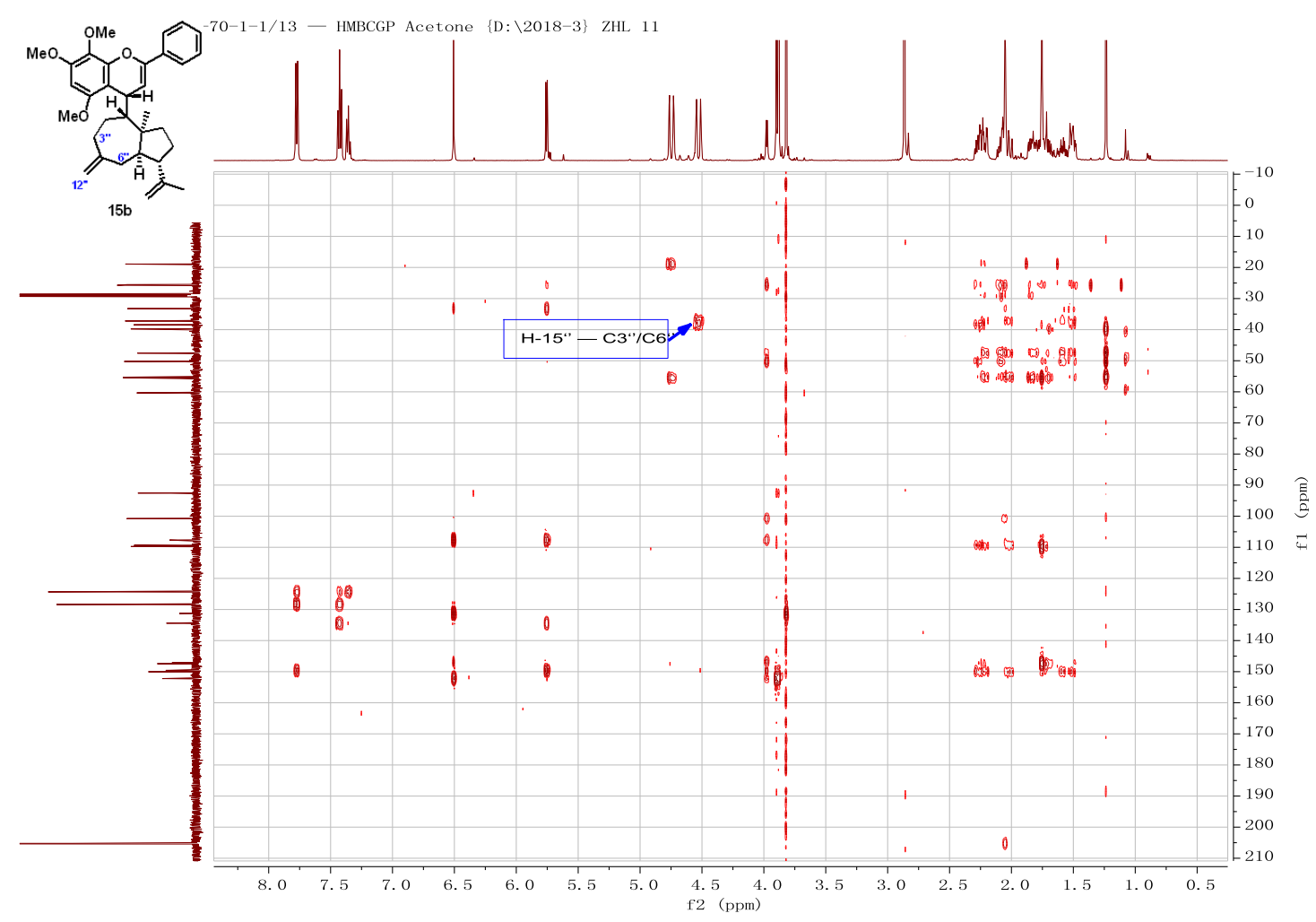

${ }^{1} \mathrm{H}-{ }^{13} \mathrm{C}$ HMBC (500 MHz \& $\left.126 \mathrm{MHz}\right)$ of Compound $15 \mathrm{~b}$ in acetone- $d_{6}$ 

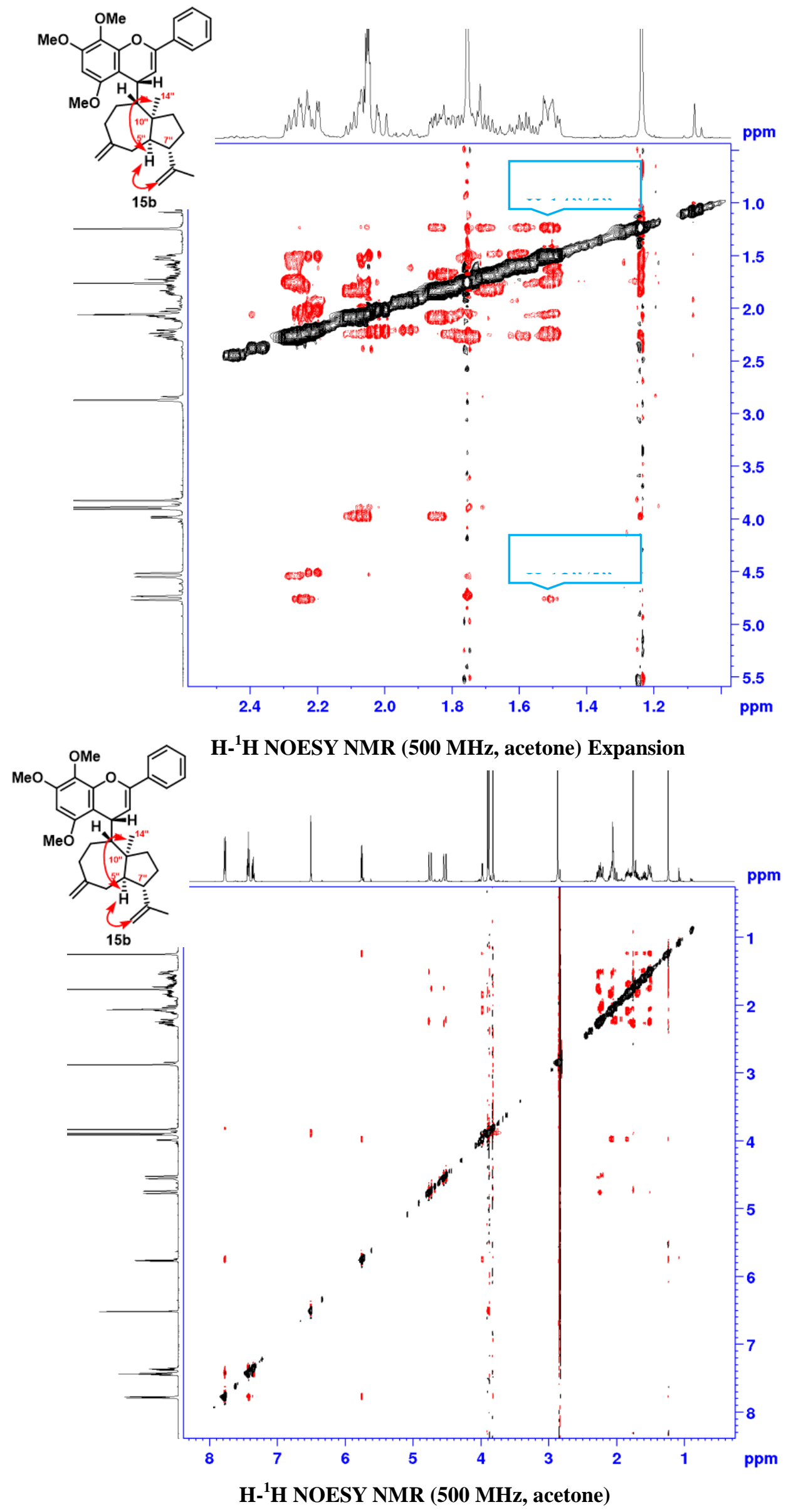

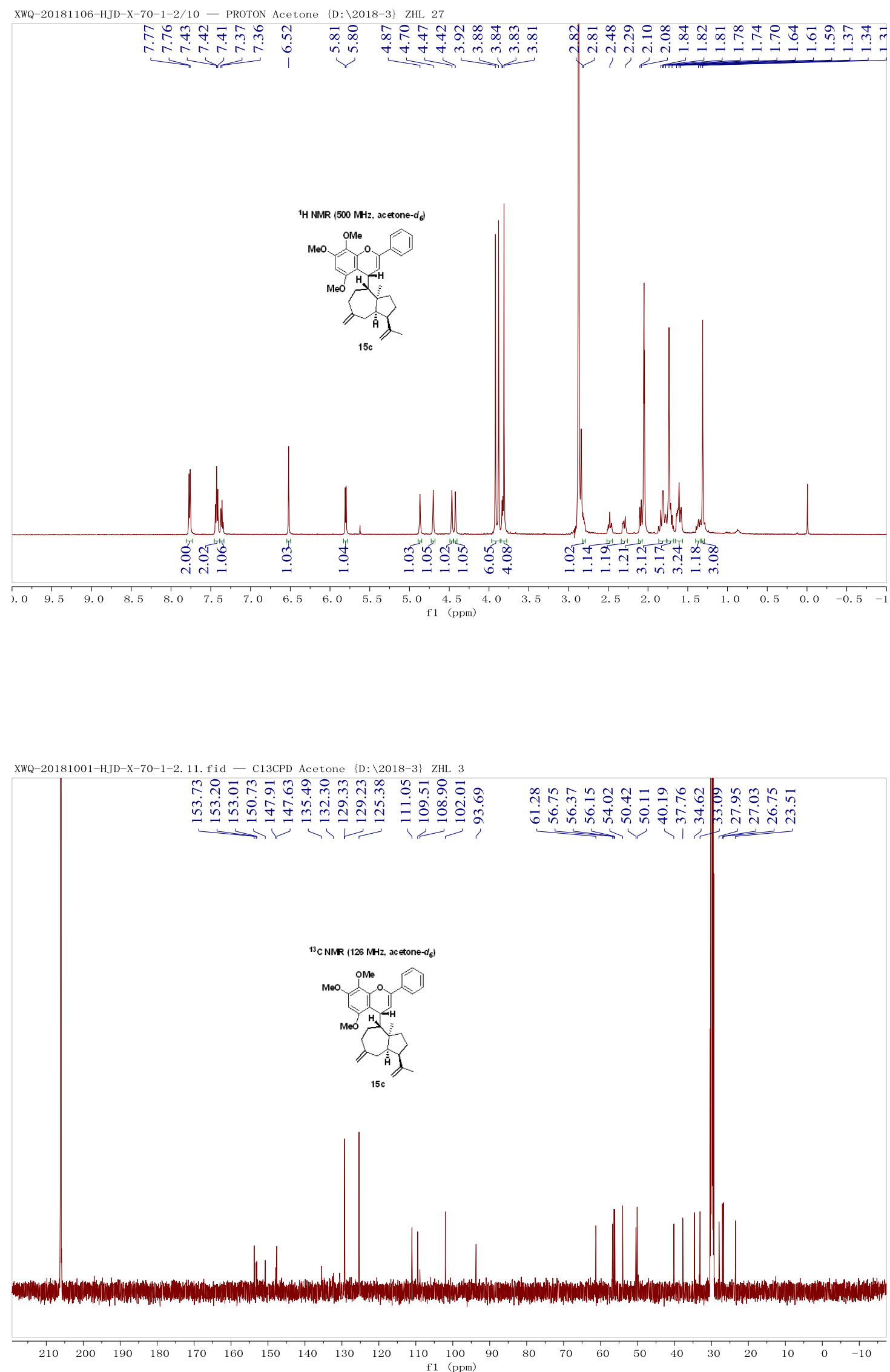


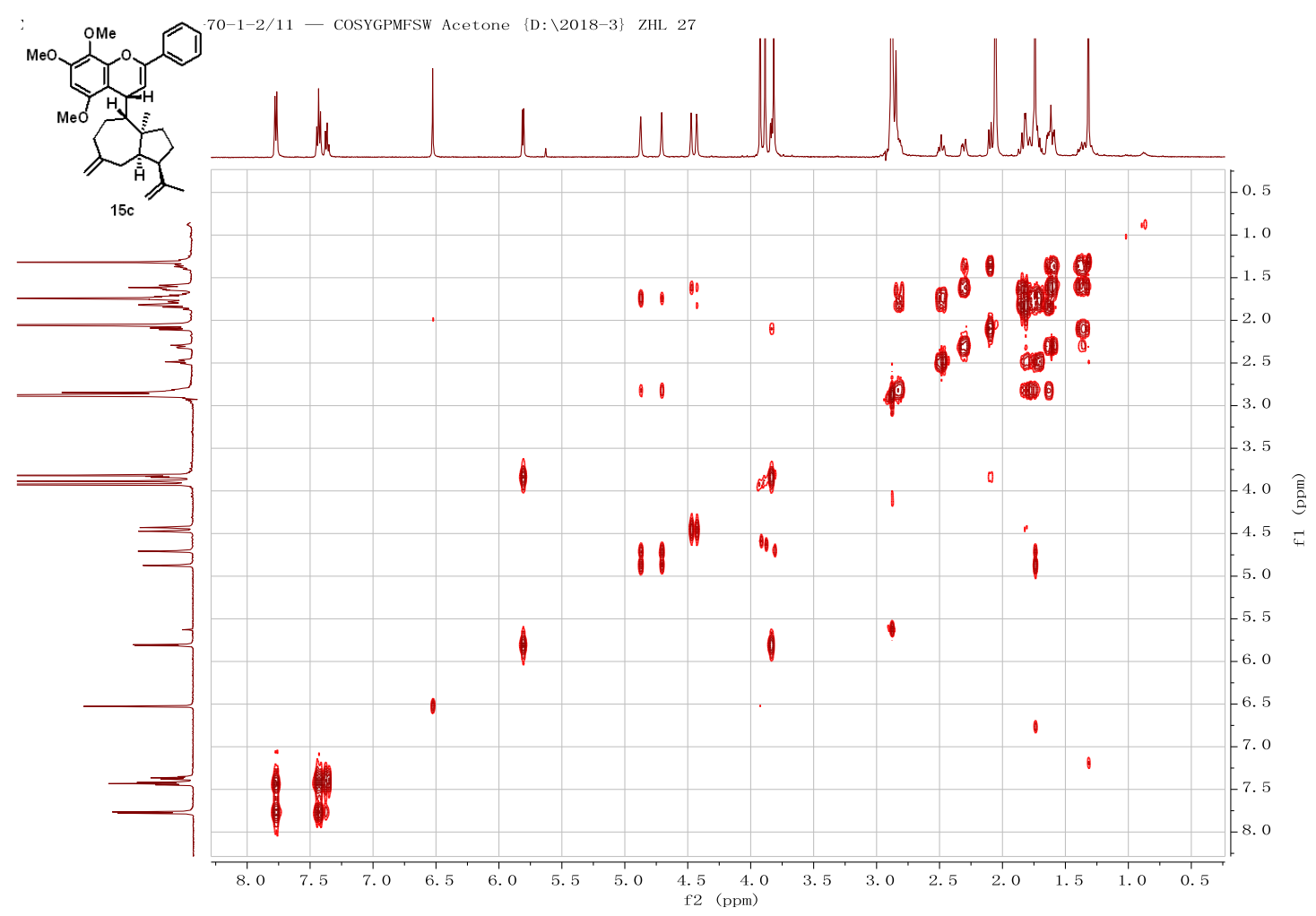

H- ${ }^{1} \mathrm{H}$ COSY NMR (500 MHz, acetone)

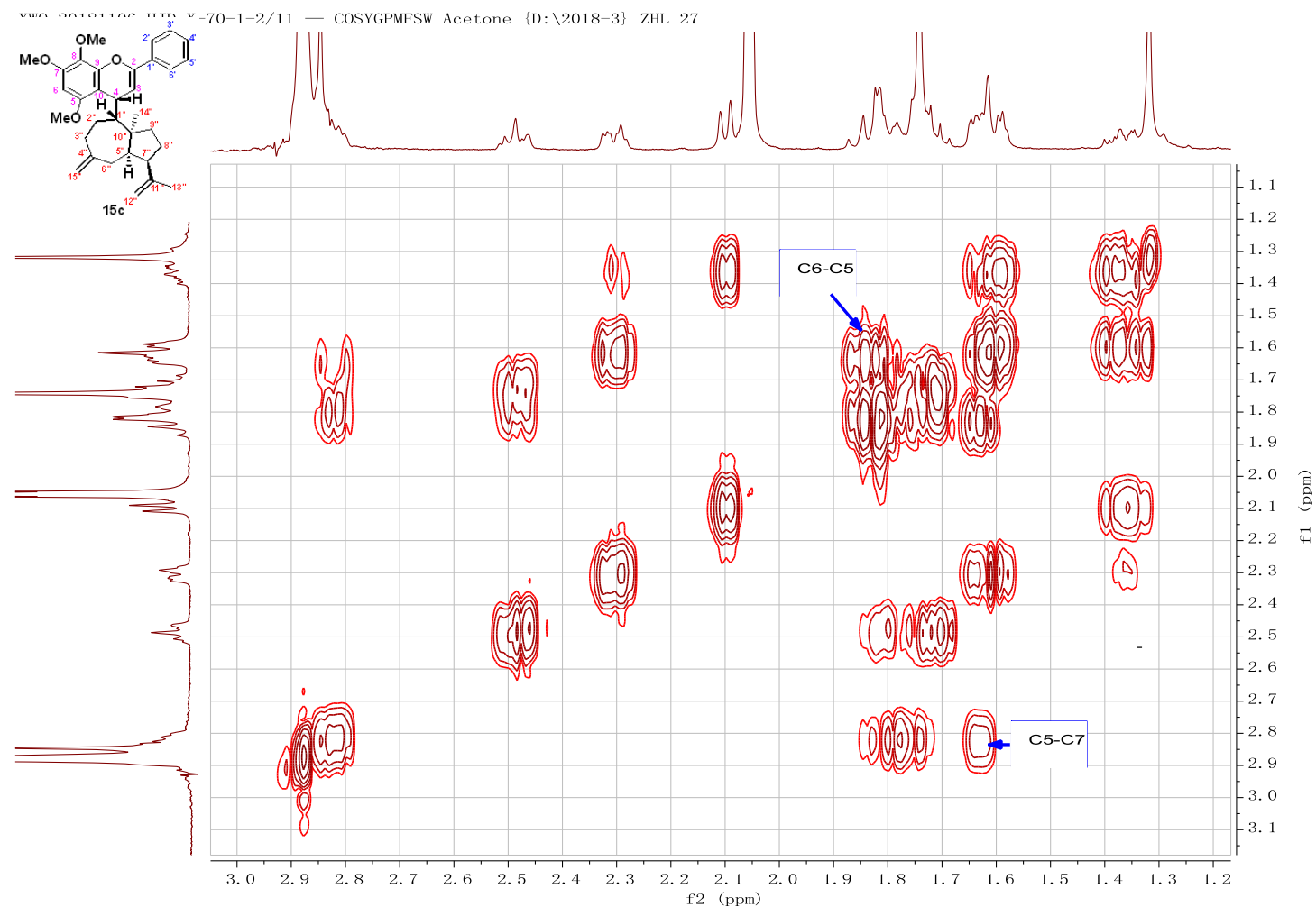

H- ${ }^{1} \mathrm{H}$ COSY NMR (500 MHz, acetone) Expansion 


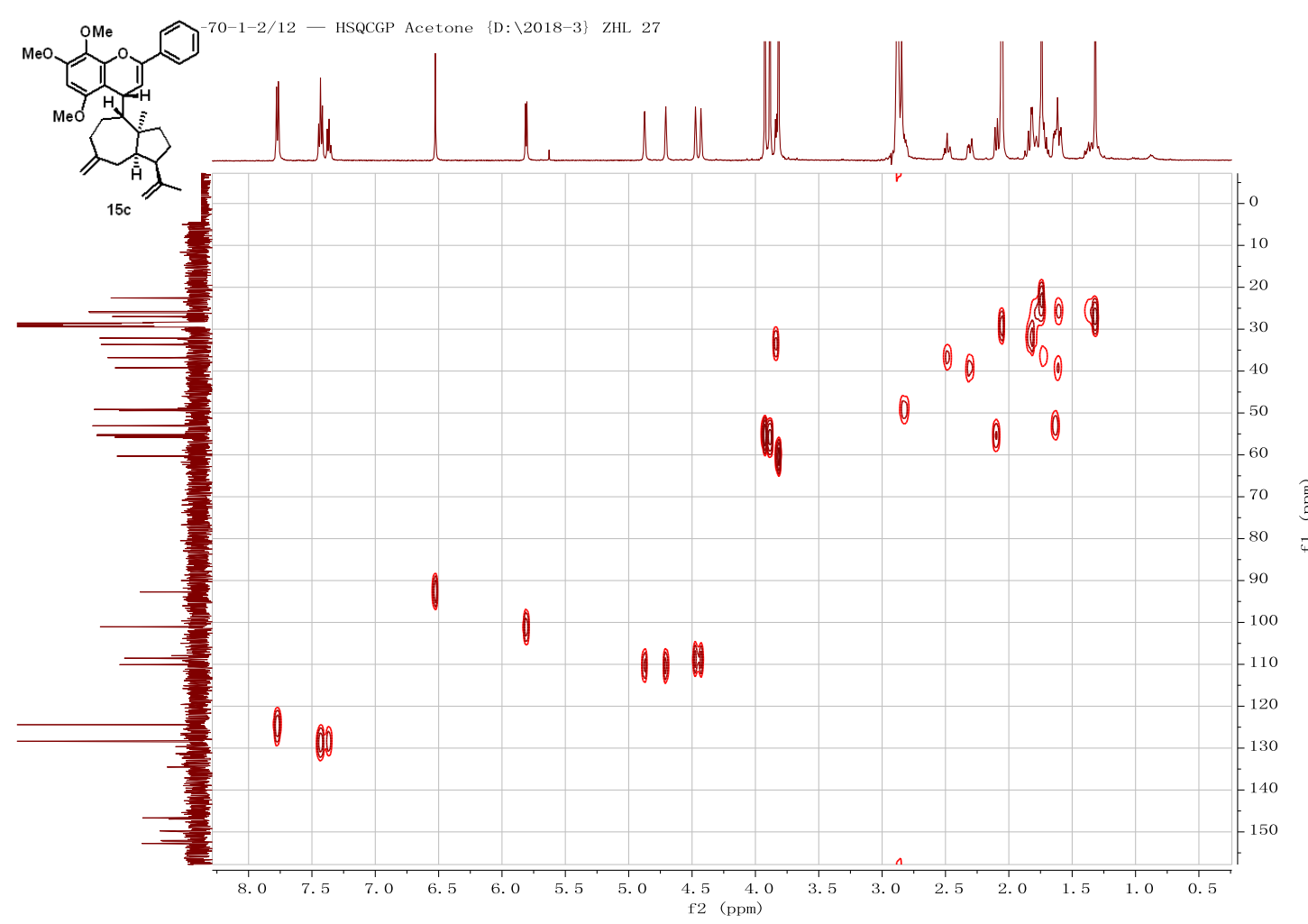

${ }^{1} \mathrm{H}-{ }^{13} \mathrm{C}$ HSQC (500 MHz \& $\left.126 \mathrm{MHz}\right)$ of Compound $15 \mathrm{c}$ in acetone- $d_{6}$

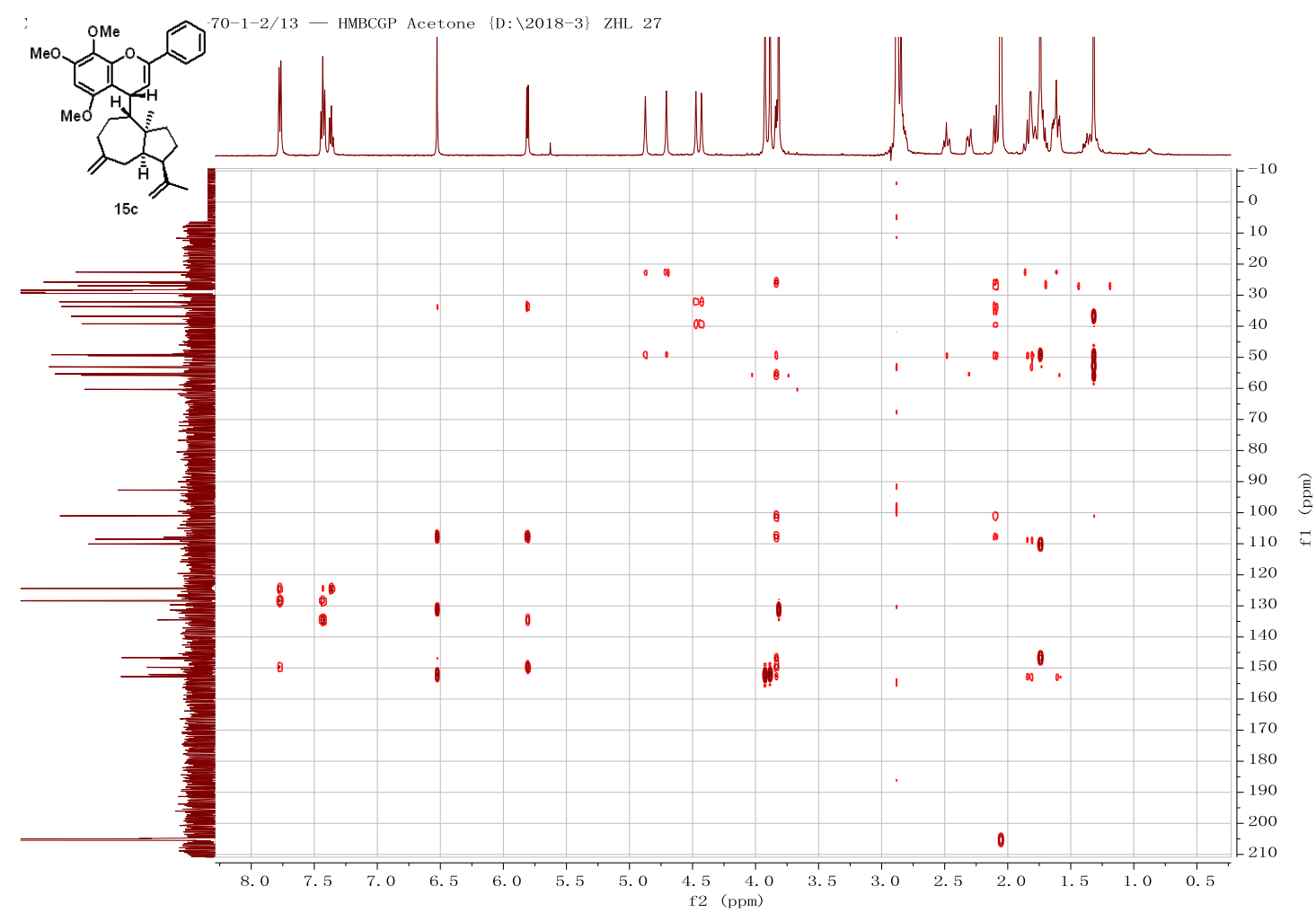

${ }^{1} \mathrm{H}-{ }^{13} \mathrm{C}$ HMBC (500 MHz \& $\left.126 \mathrm{MHz}\right)$ of Compound 15c in acetone- $d_{6}$ 


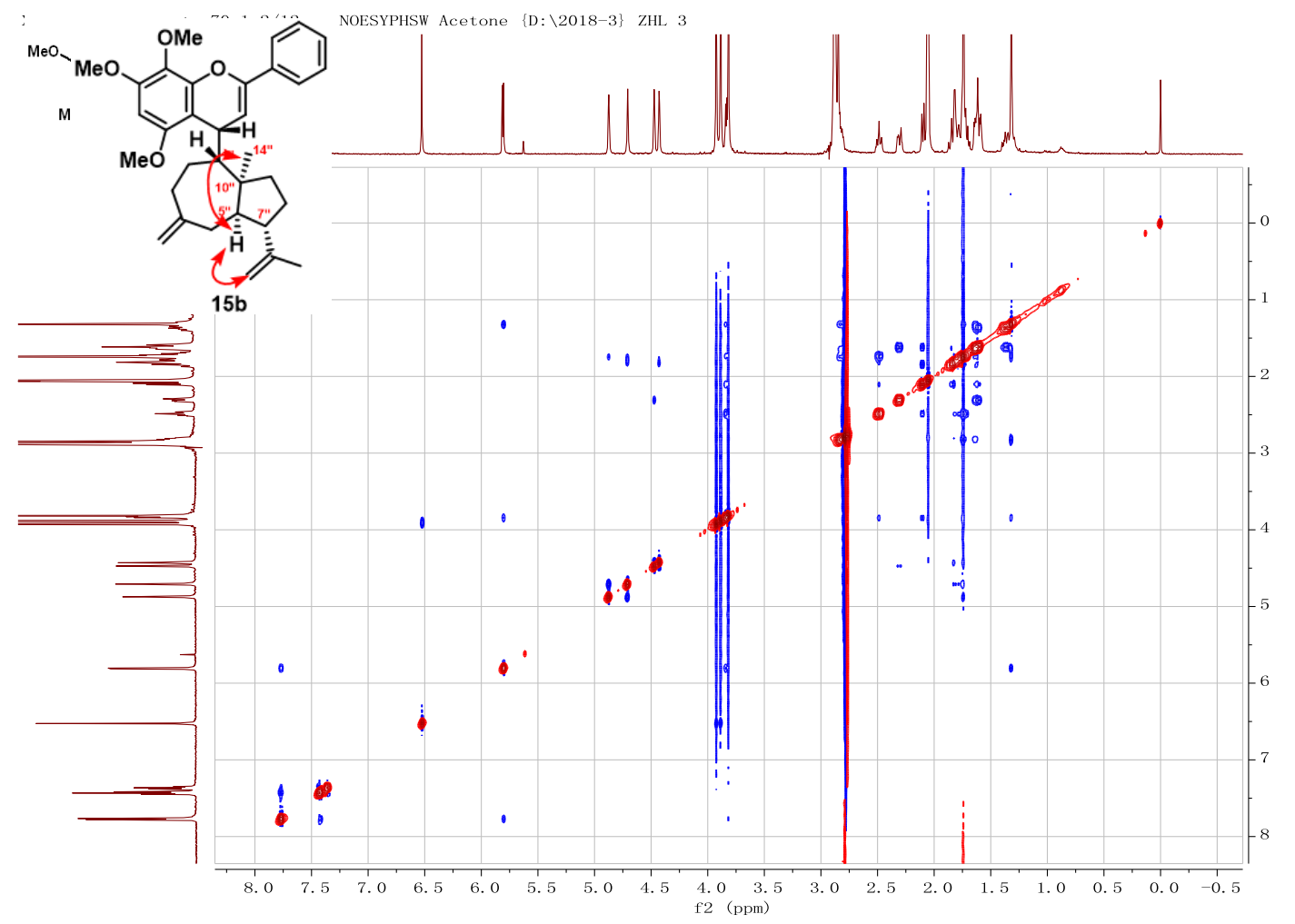

H- ${ }^{1} \mathrm{H}$ NOESY NMR (500 MHz, acetone)

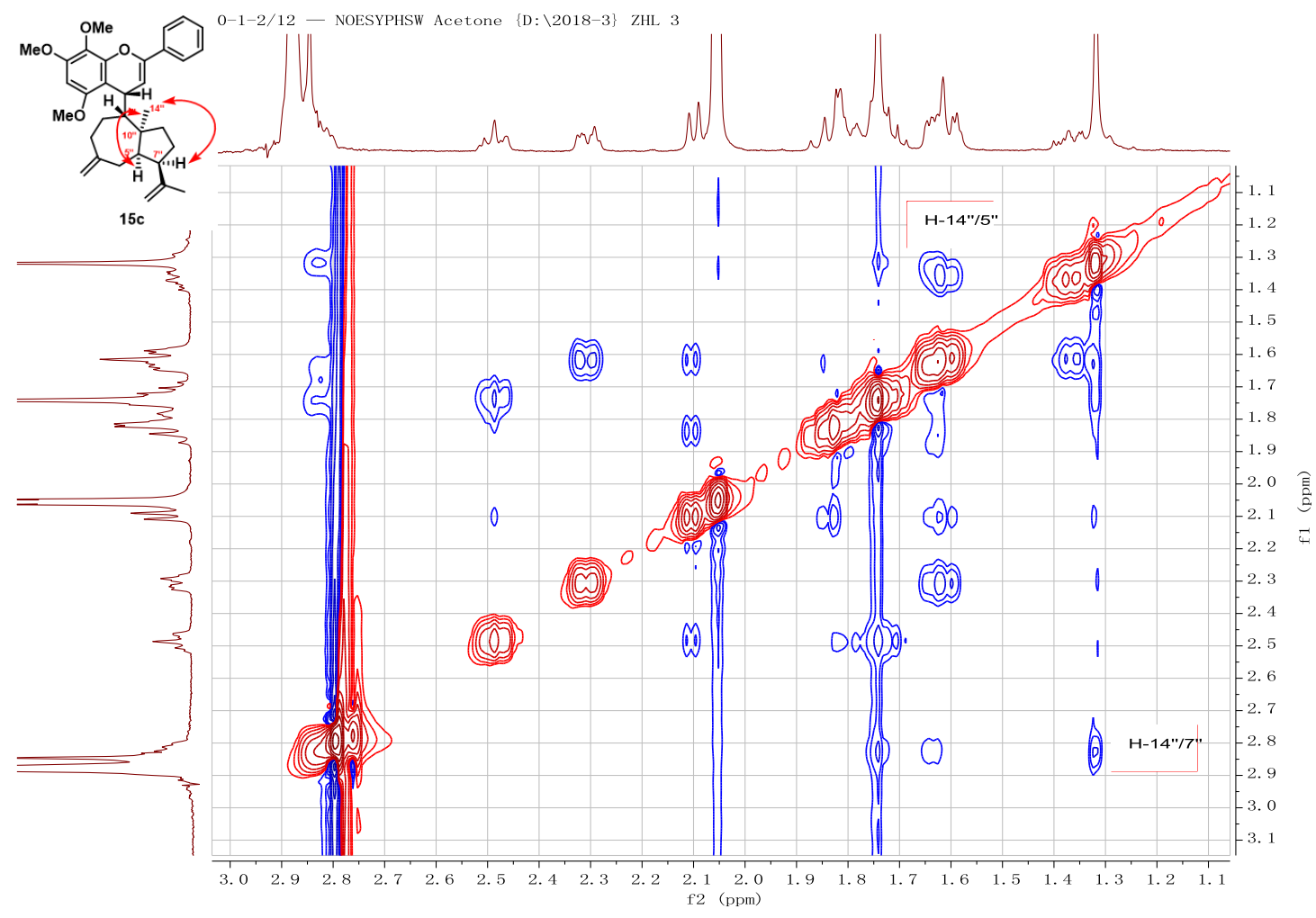

H- ${ }^{1} \mathrm{H}$ NOESY NMR (500 MHz, acetone) Expansion 


\section{References}

1. Jiadong Hu, Yu-Qi Gao, Dongyang Xu, Le Chen, Wen Wen, Yi Hou, Lu Chen, Weiqing Xie. Chem. Commun., 2020, 56, 10018-10021.

2. Nyhlén, J.; Eriksson, L.; Bäckvall, J. Chirality. 2008, 20, 47-50.

3. Porzel, A.; Phuong Lien, T.; Schmidt, J.; Drosihn, S.; Wagner, C.; Merzweiler, K.; Van Sung, T.; Adam, G. Tetrahedron. 2000, 56, 865-872 


\section{HPLC Charts for ent-10}

HPLC Report

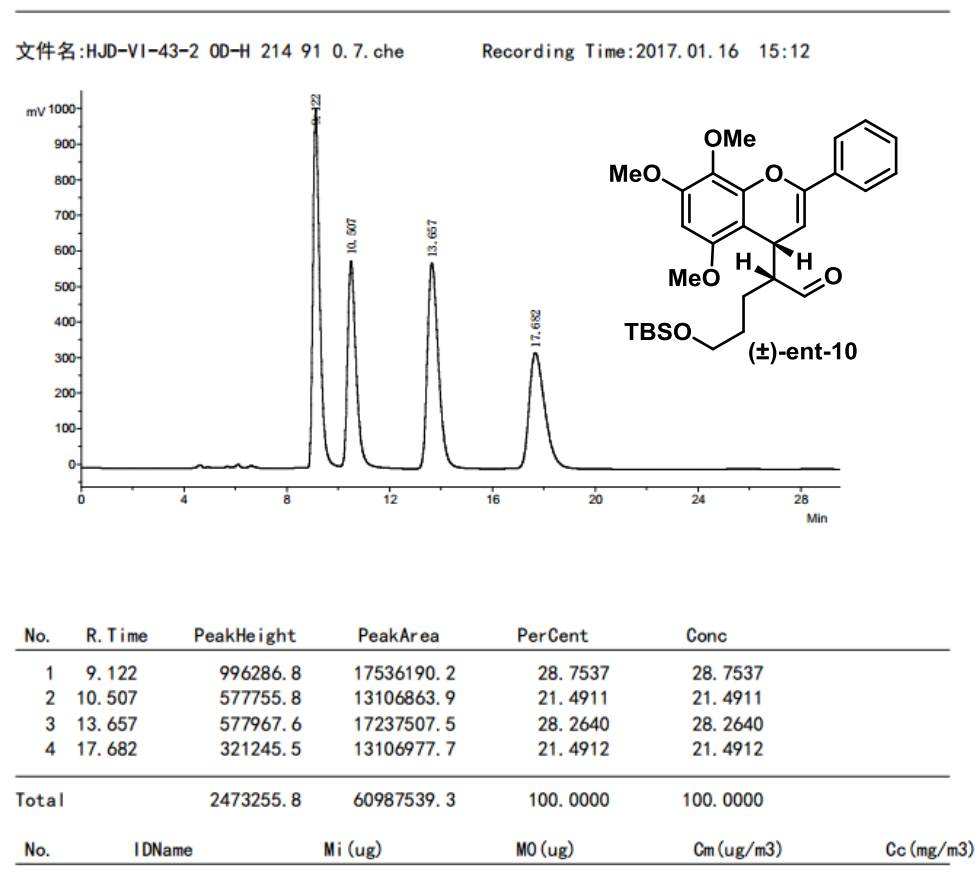

HPLC Report

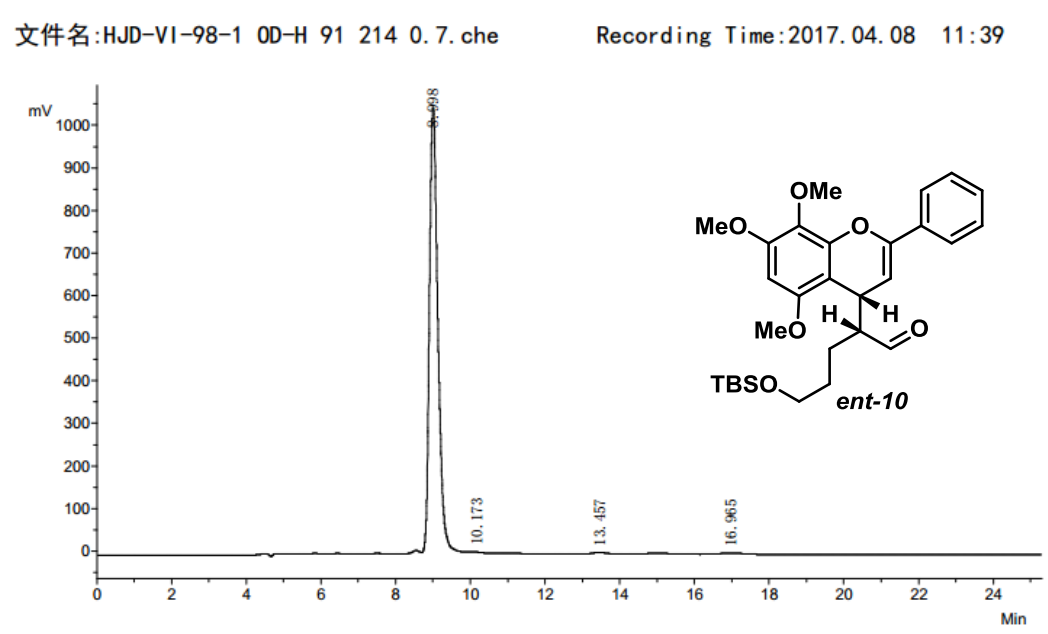

\begin{tabular}{|c|c|c|c|c|c|c|}
\hline No. & R. Time & PeakHe ight & PeakArea & PerCent & Conc & $>20: 1$ \\
\hline 1 & 8. 998 & 1043875.0 & 16455030. 1 & 98.4380 & 98.4380 & \\
\hline 2 & 10.173 & 2189.6 & 50618. 7 & 0. 3028 & 0. 3028 & \\
\hline 3 & 13. 457 & 4053.6 & 104758.7 & 0.6267 & 0.6267 & \\
\hline 4 & 16.965 & 2991.6 & 105730.0 & 0.6325 & 0.6325 & \\
\hline Total & & 1053109.7 & 16716137.5 & 100.0000 & 100. 0000 & \\
\hline No. & \multicolumn{2}{|c|}{ IDName } & (ug) & MO (ug) & $\mathrm{Cm}$ (ug/m3) & $\mathrm{Cc}(\mathrm{mg}$ \\
\hline
\end{tabular}




\section{6. ${ }^{1} \mathrm{H} \&{ }^{13} \mathrm{C}$ NMR Spectra of compounds}
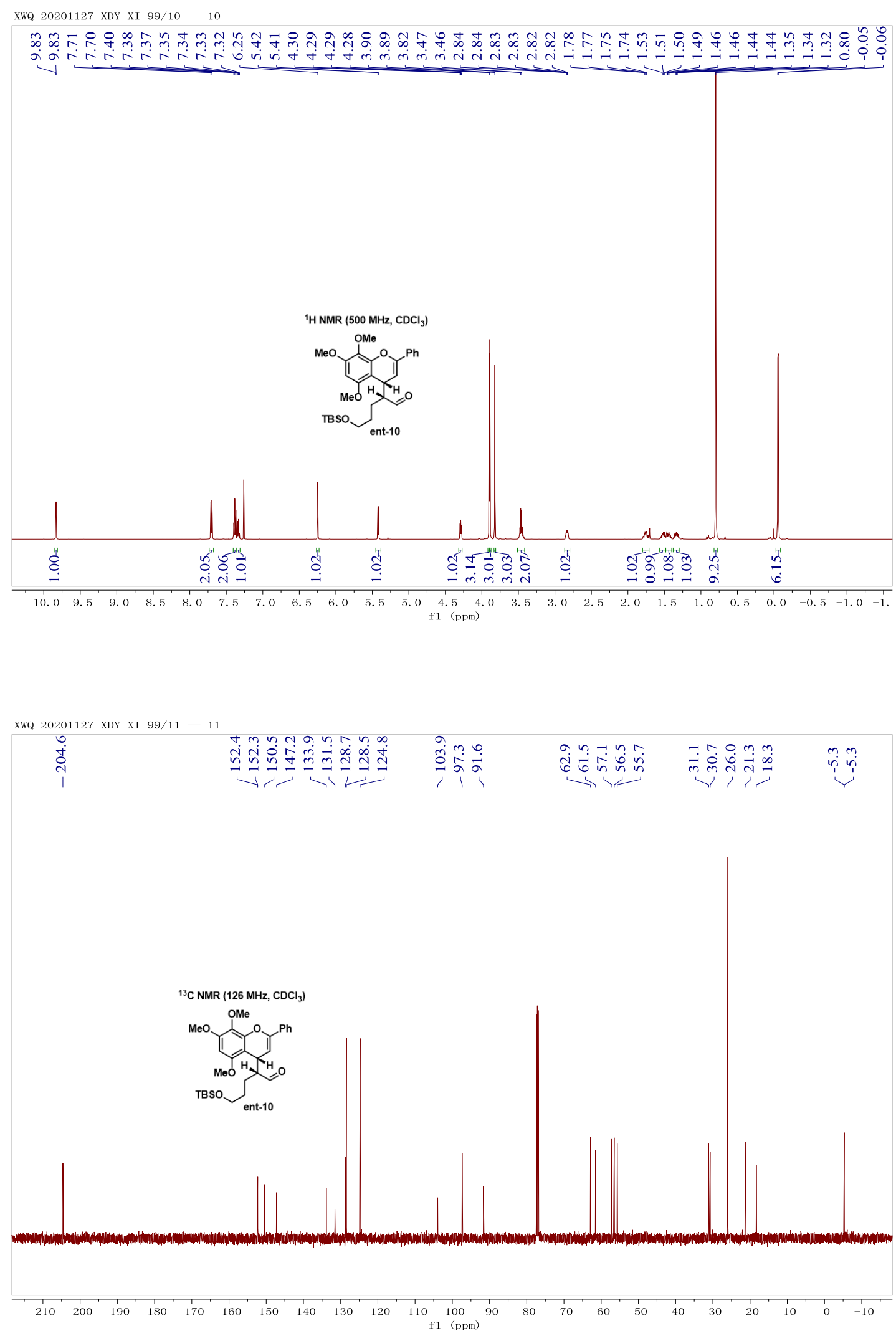


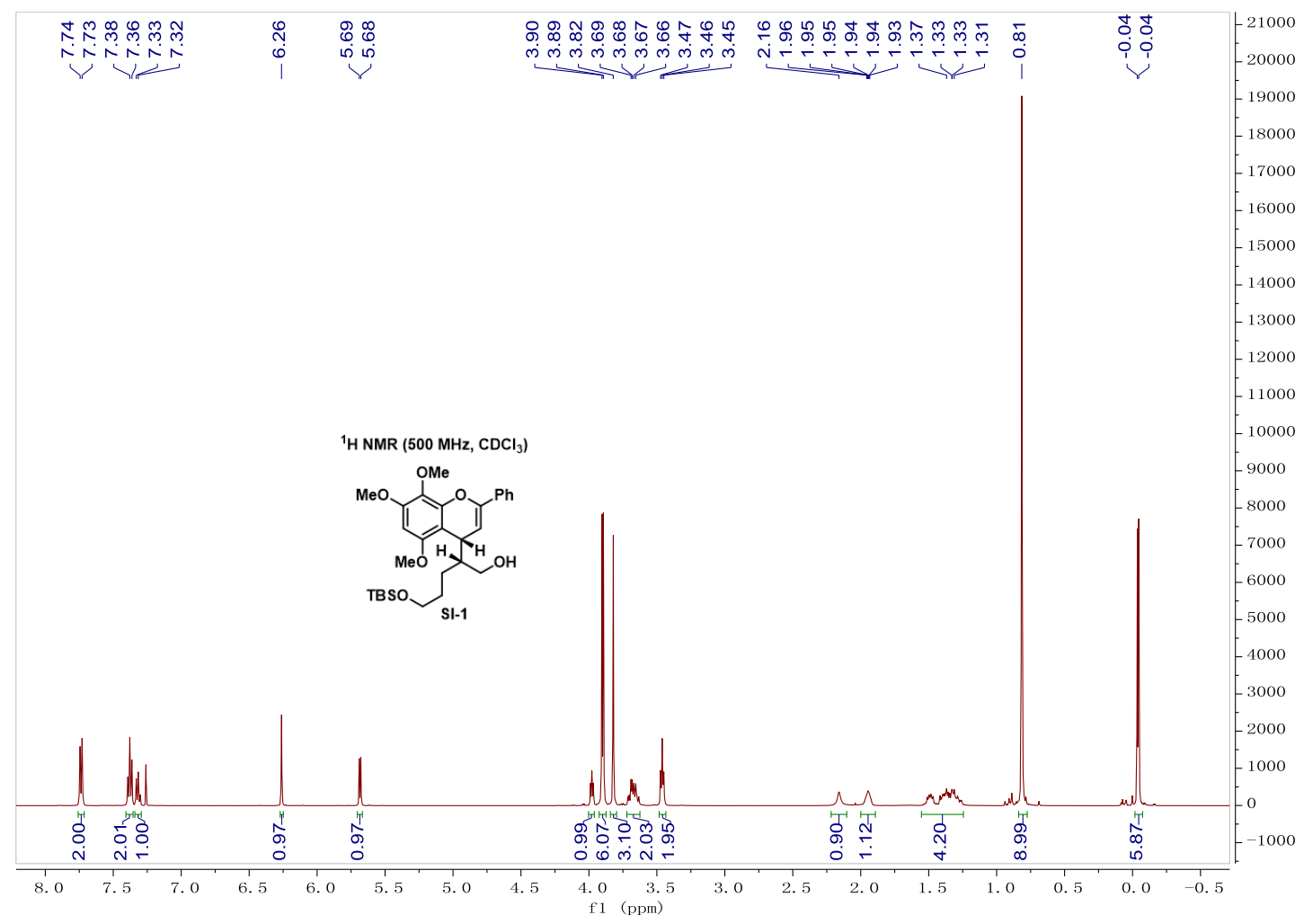

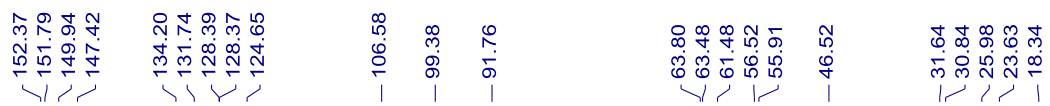
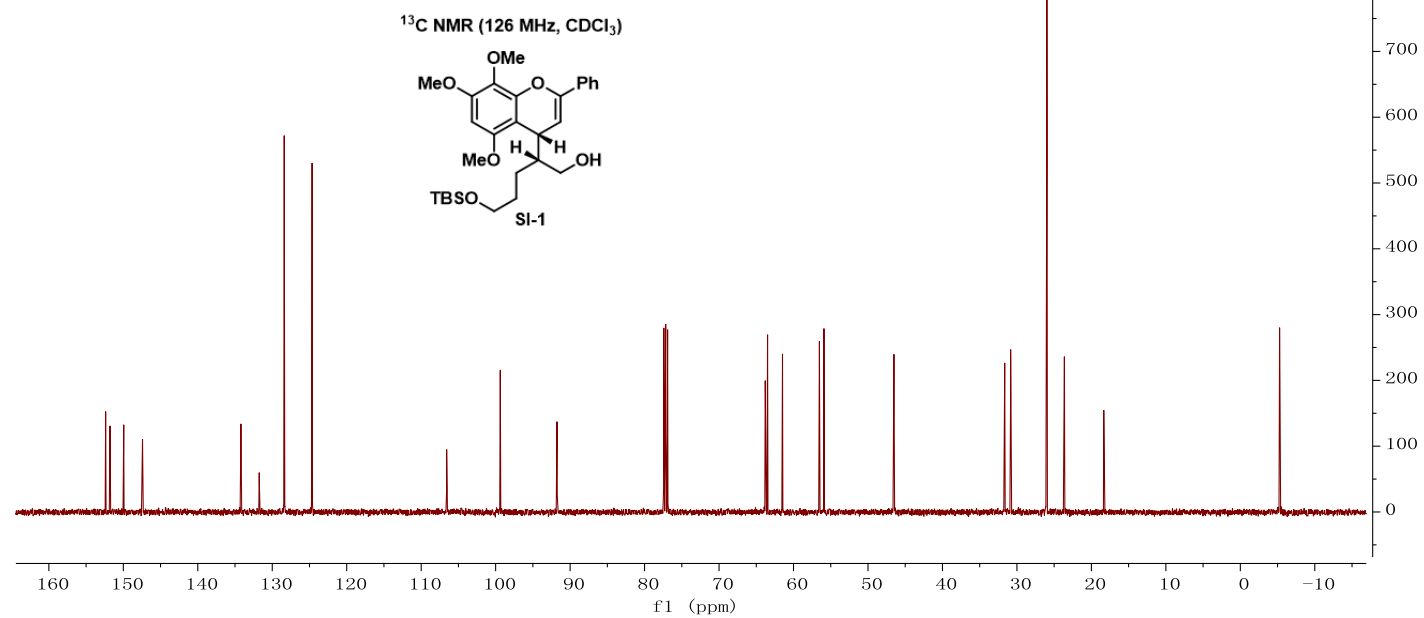


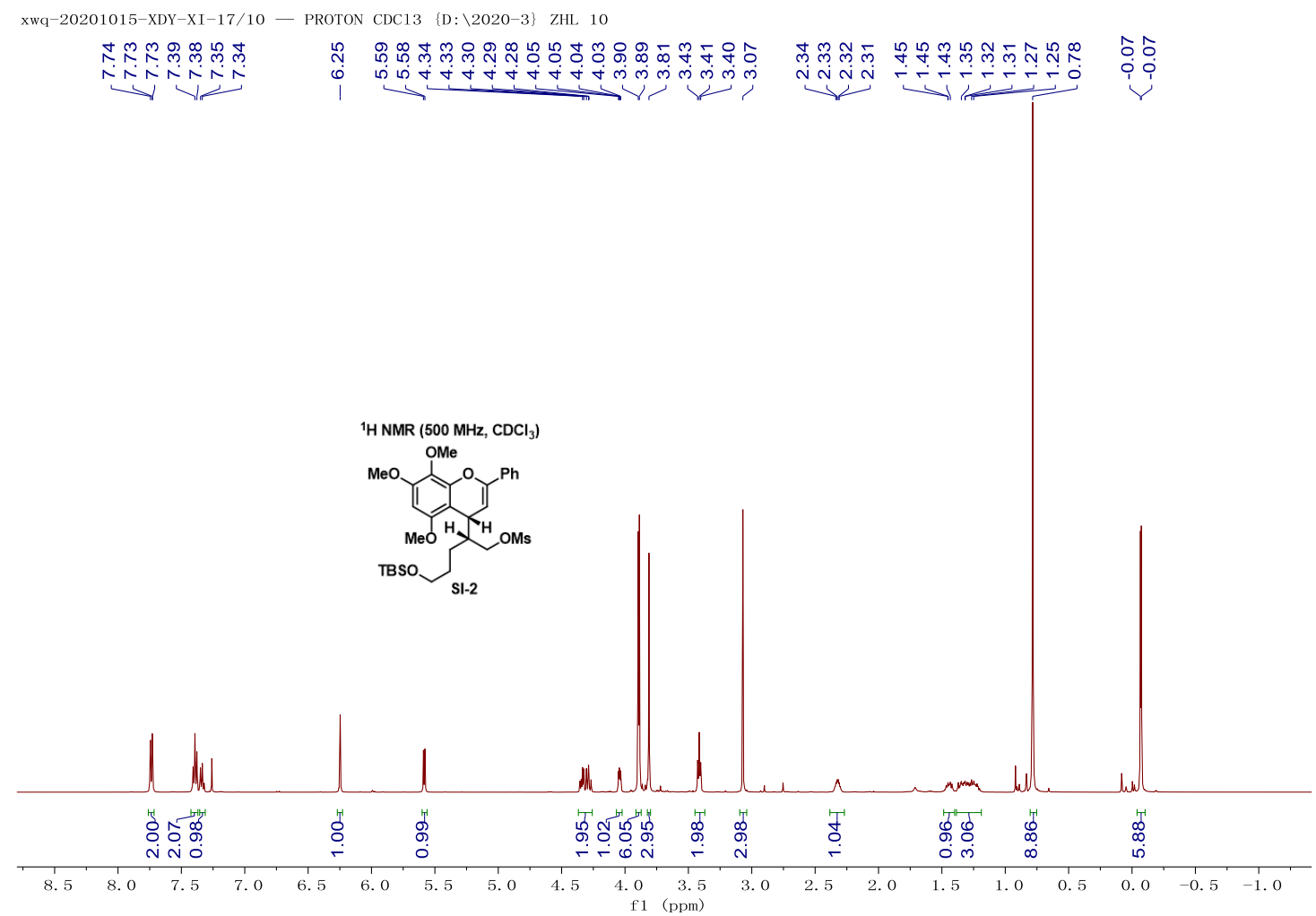

xwq-20201015-XDY-XI-17/12-C13CPD CDC13 \{D: \2020-3\} ZHL 10

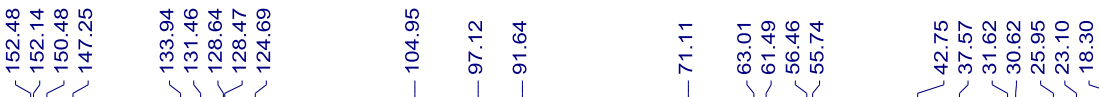

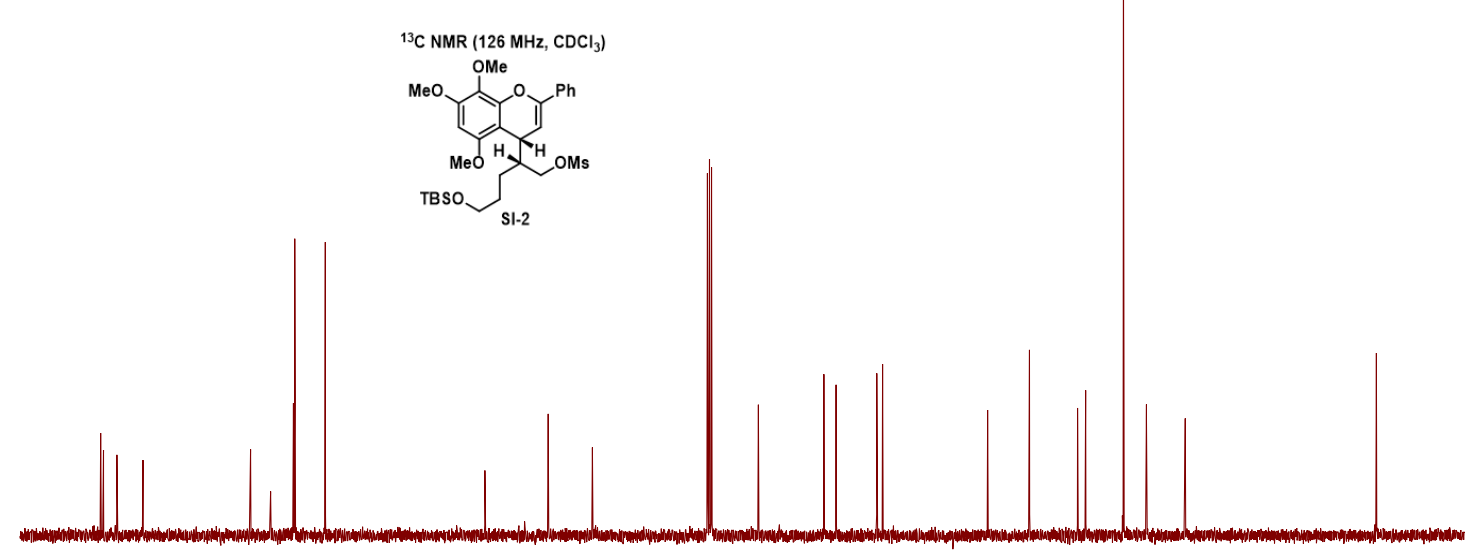

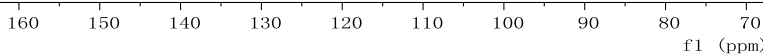



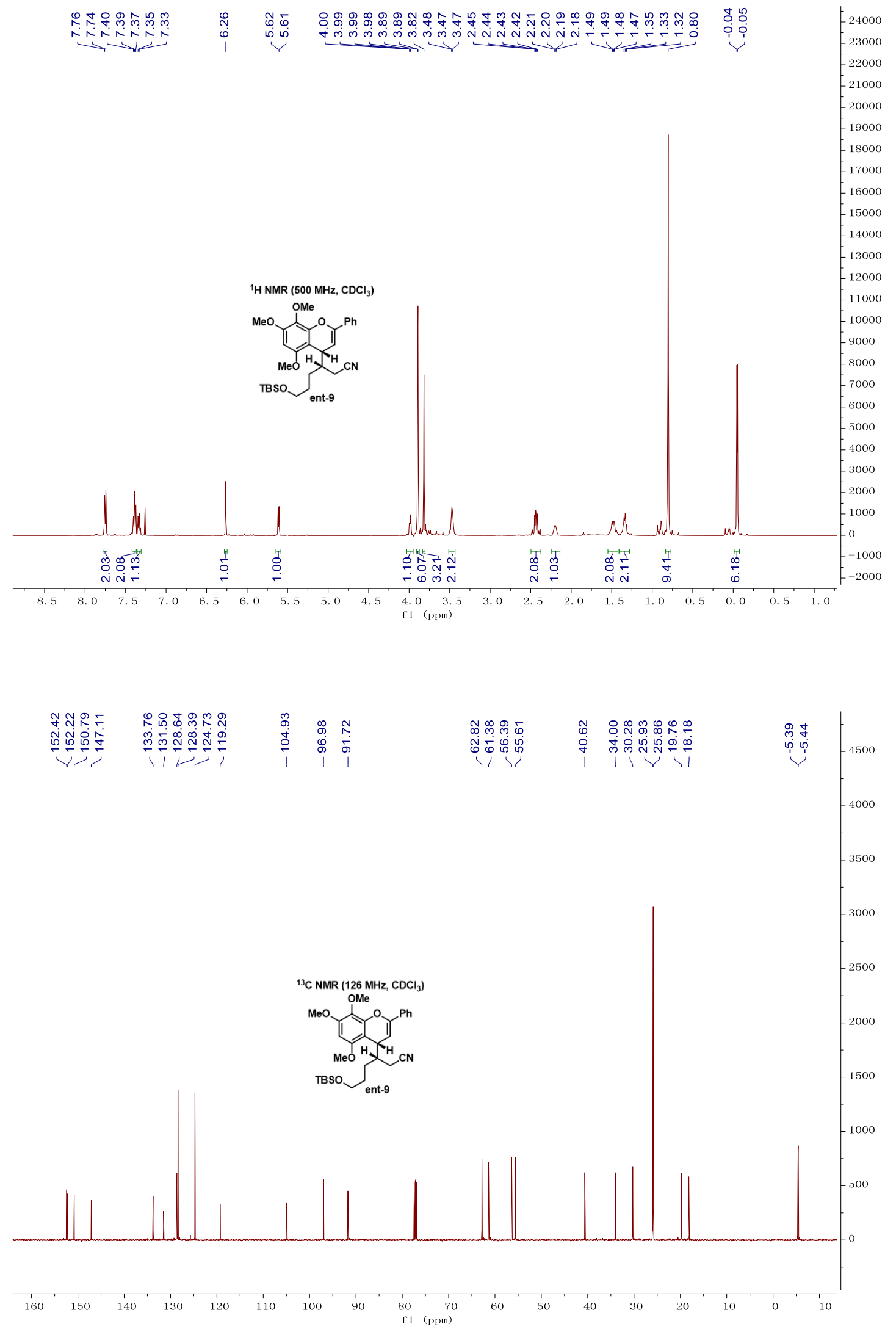


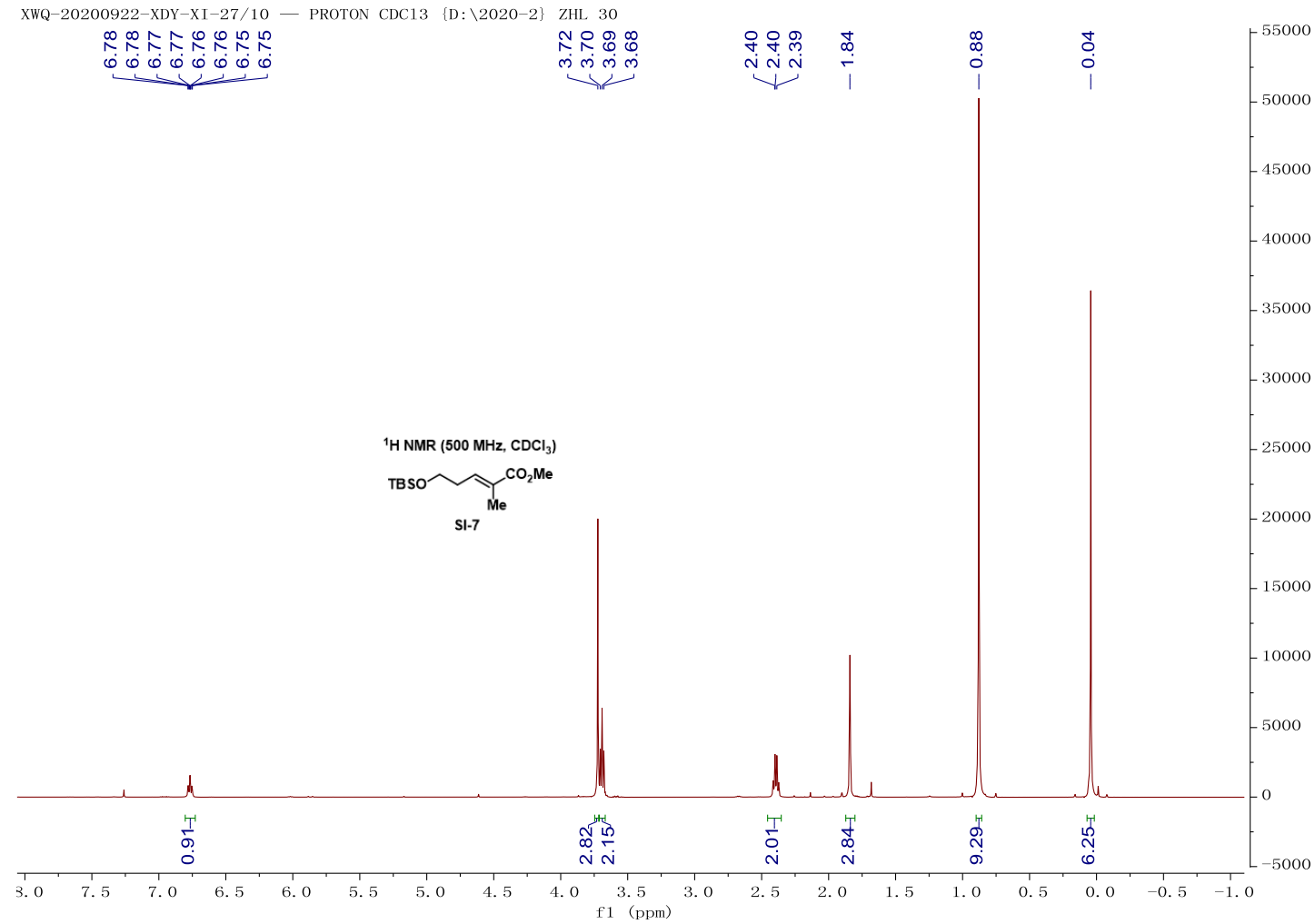

XWQ-20200922-XDY-XI-27/11-C13CPD CDC13 \{D:\2020-2\} ZHL 30

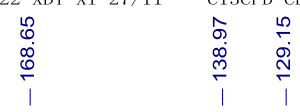

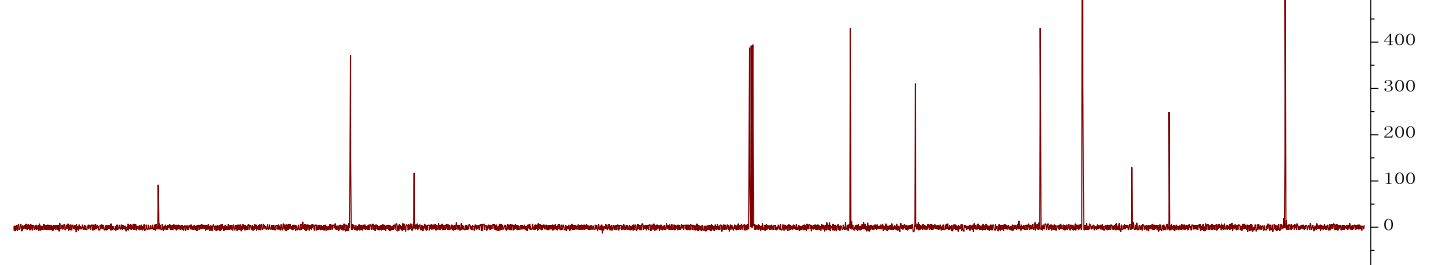




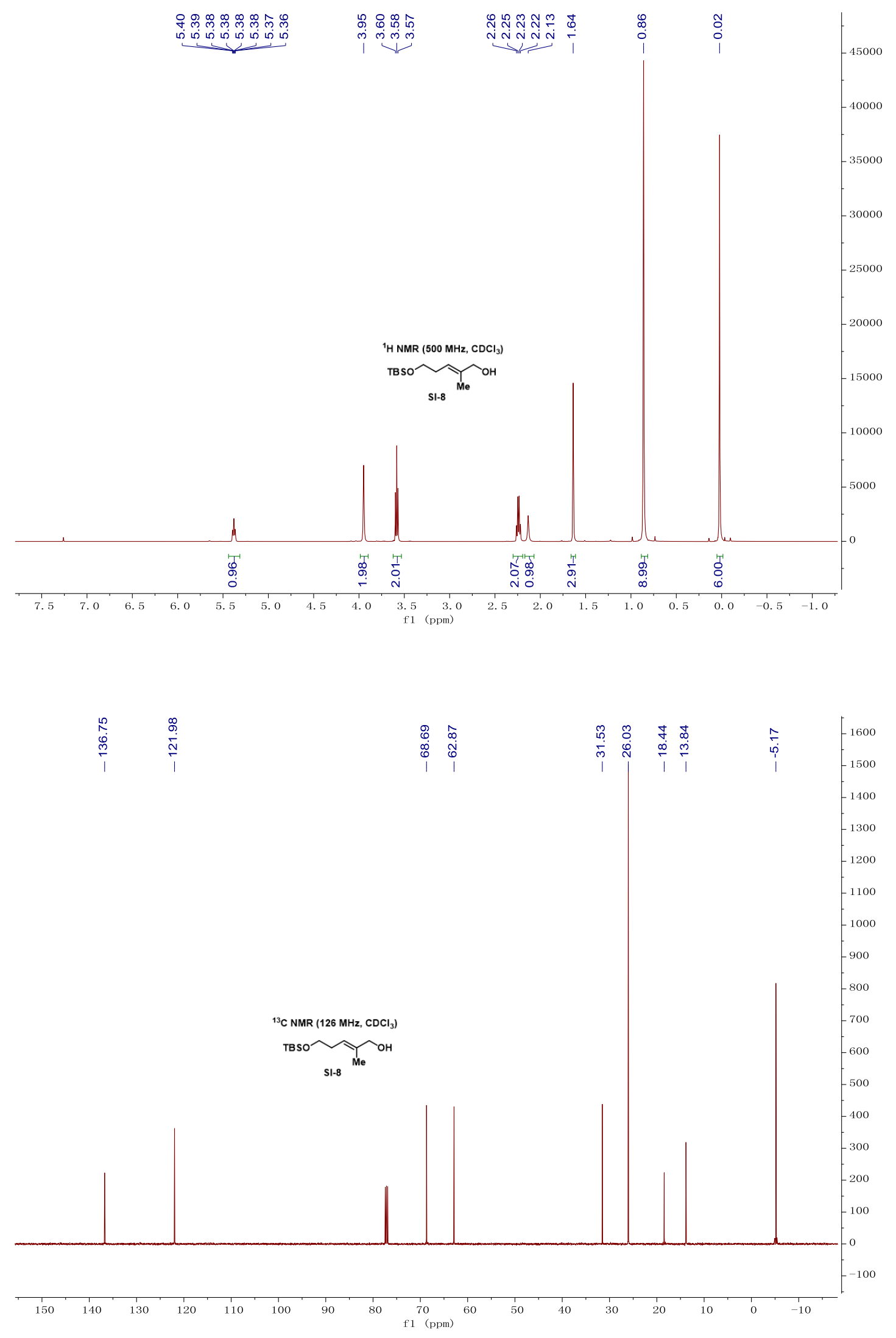



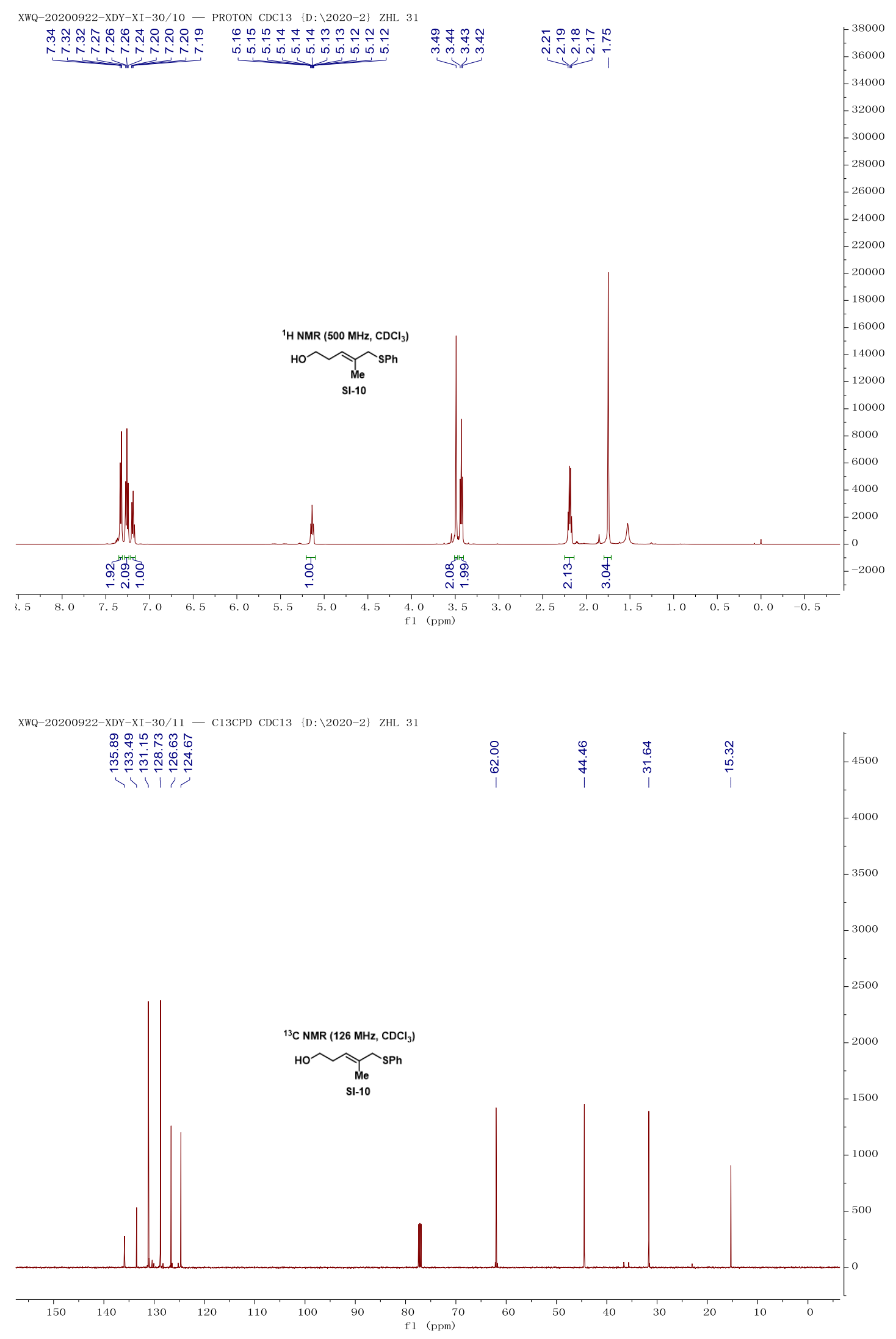

46 

TsO

Me
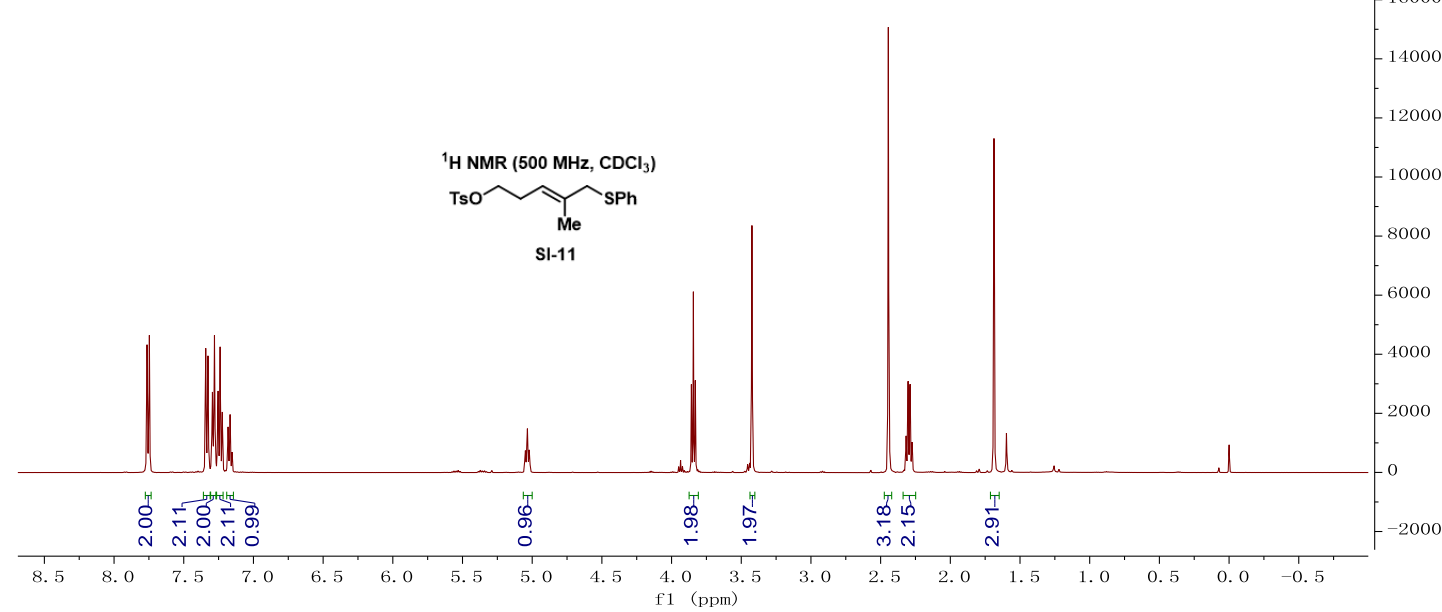

XWQ-20200922-XDY-XI-31/11-C13CPD CDC13 $\{\mathrm{D}: \backslash 2020-2\} \quad$ ZHL 32 ๙

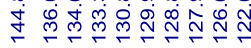
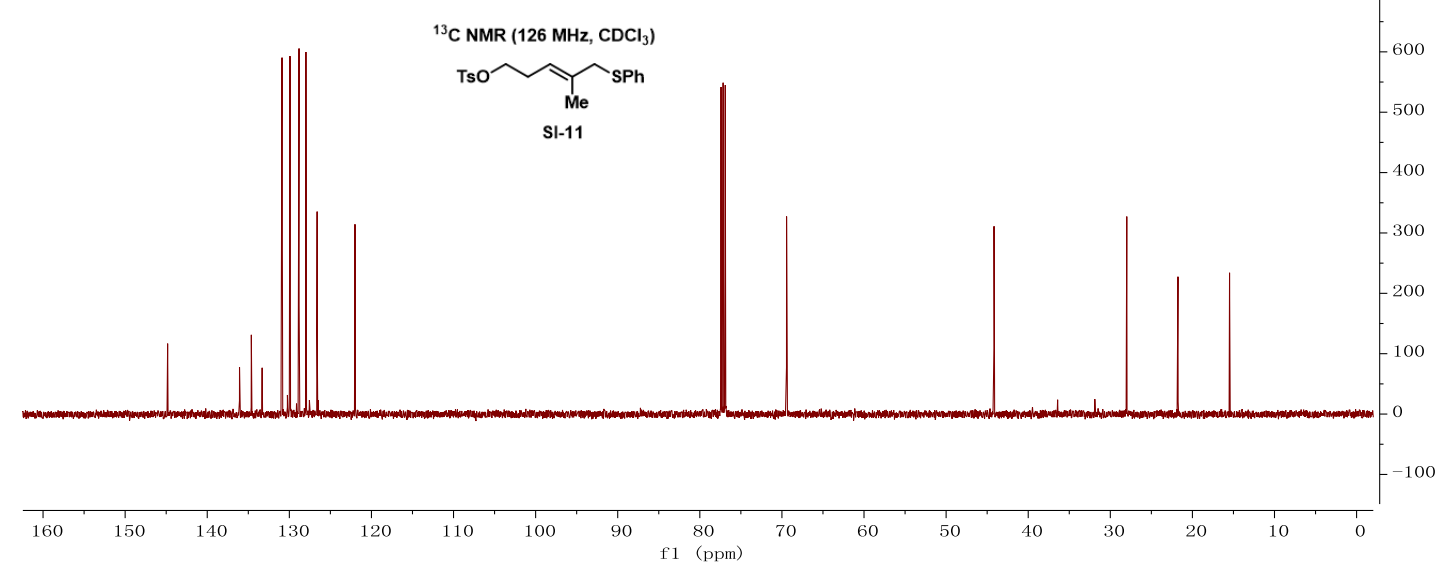


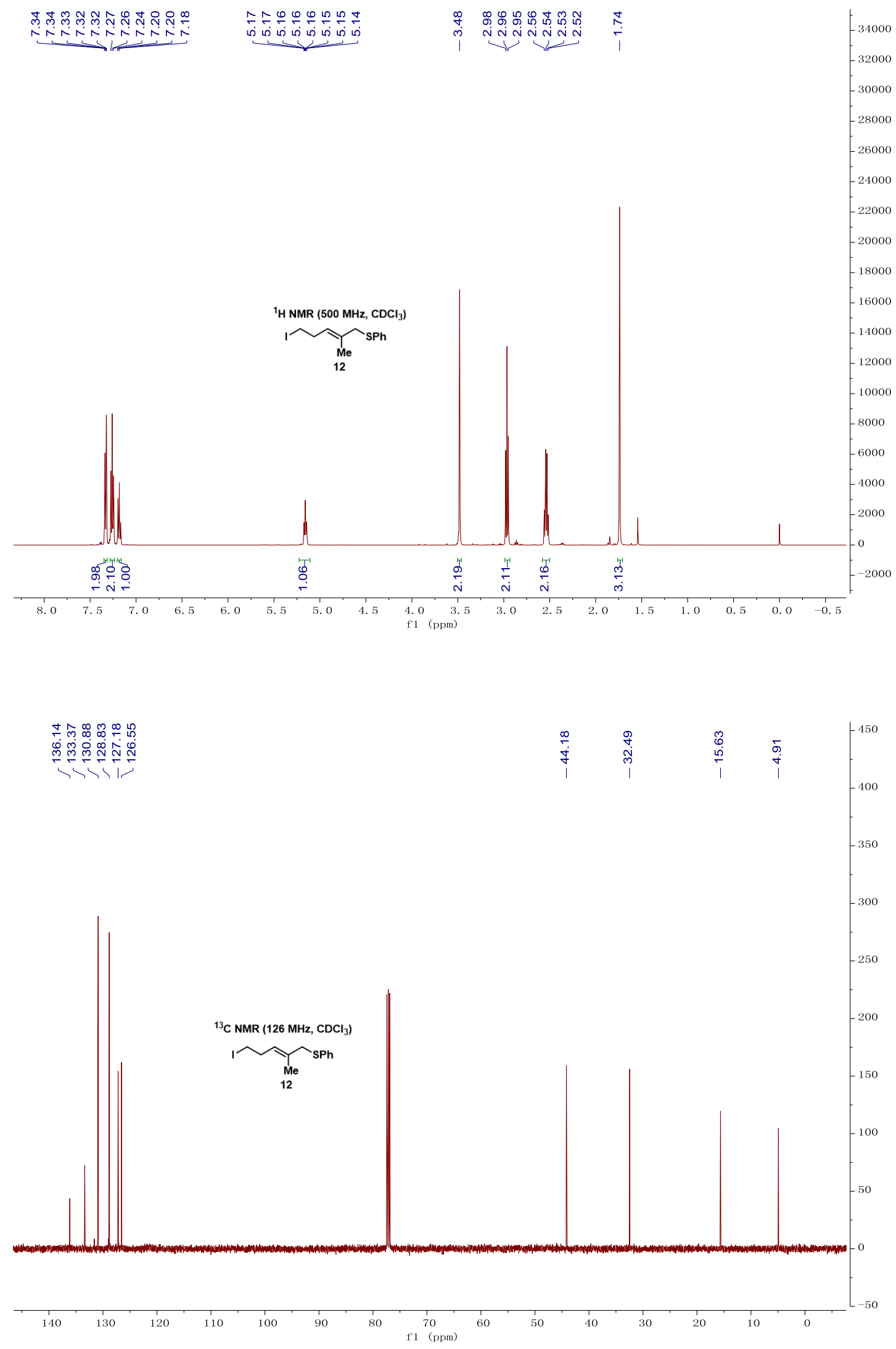




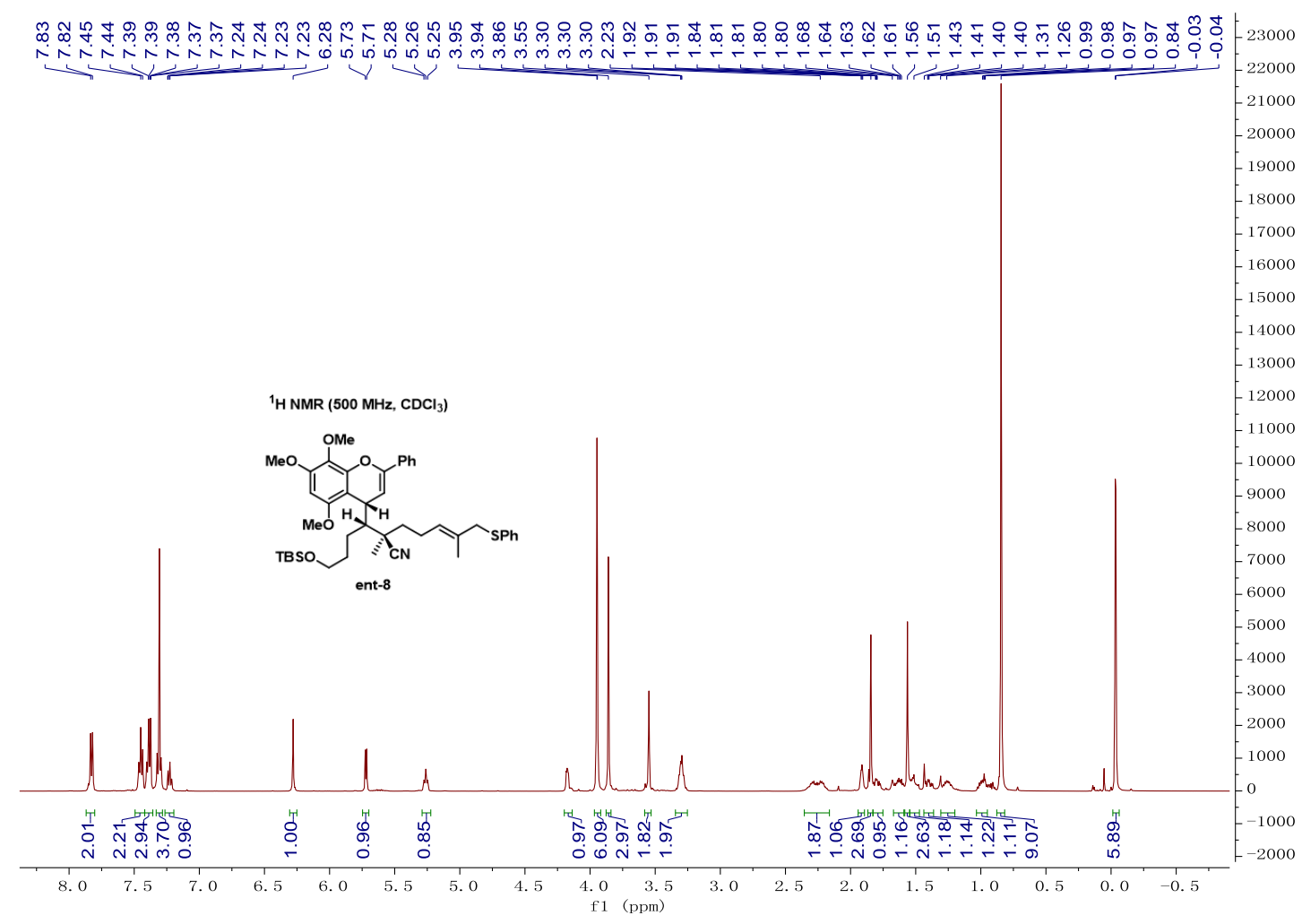

品

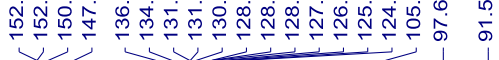

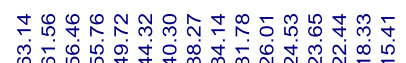

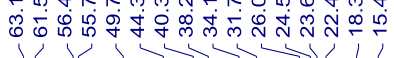

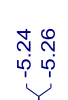

$-1200$

$\left.{ }^{13} \mathrm{C} \mathrm{NMR} \mathrm{(126} \mathrm{MHz,} \mathrm{CDCl}_{3}\right)$
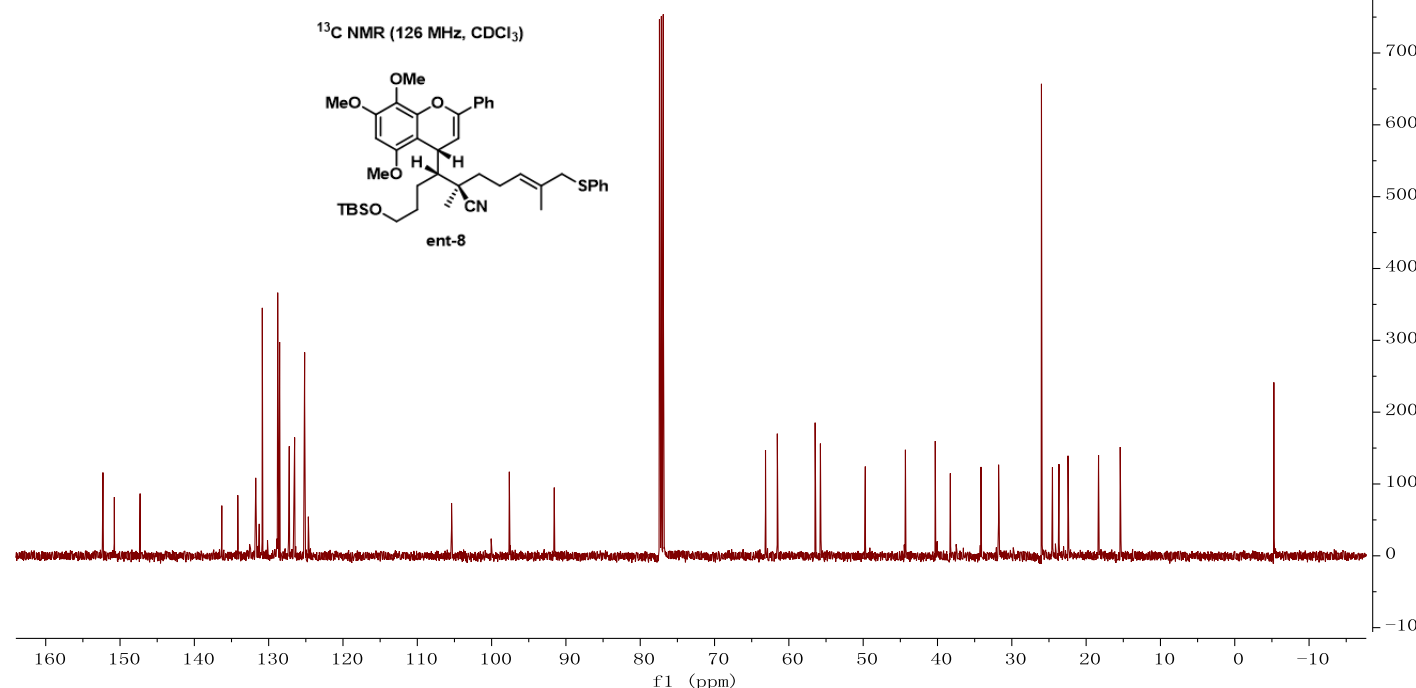


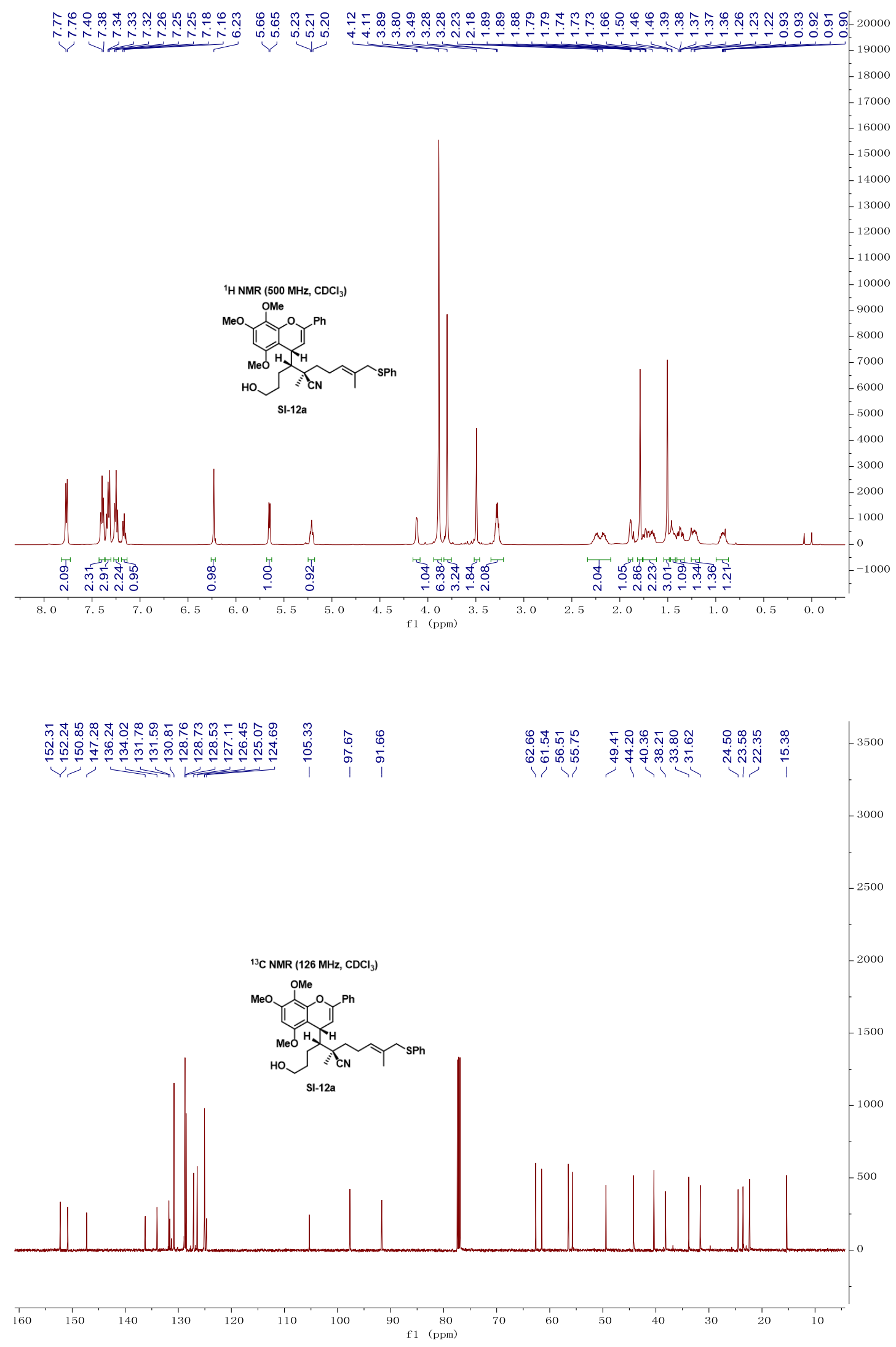




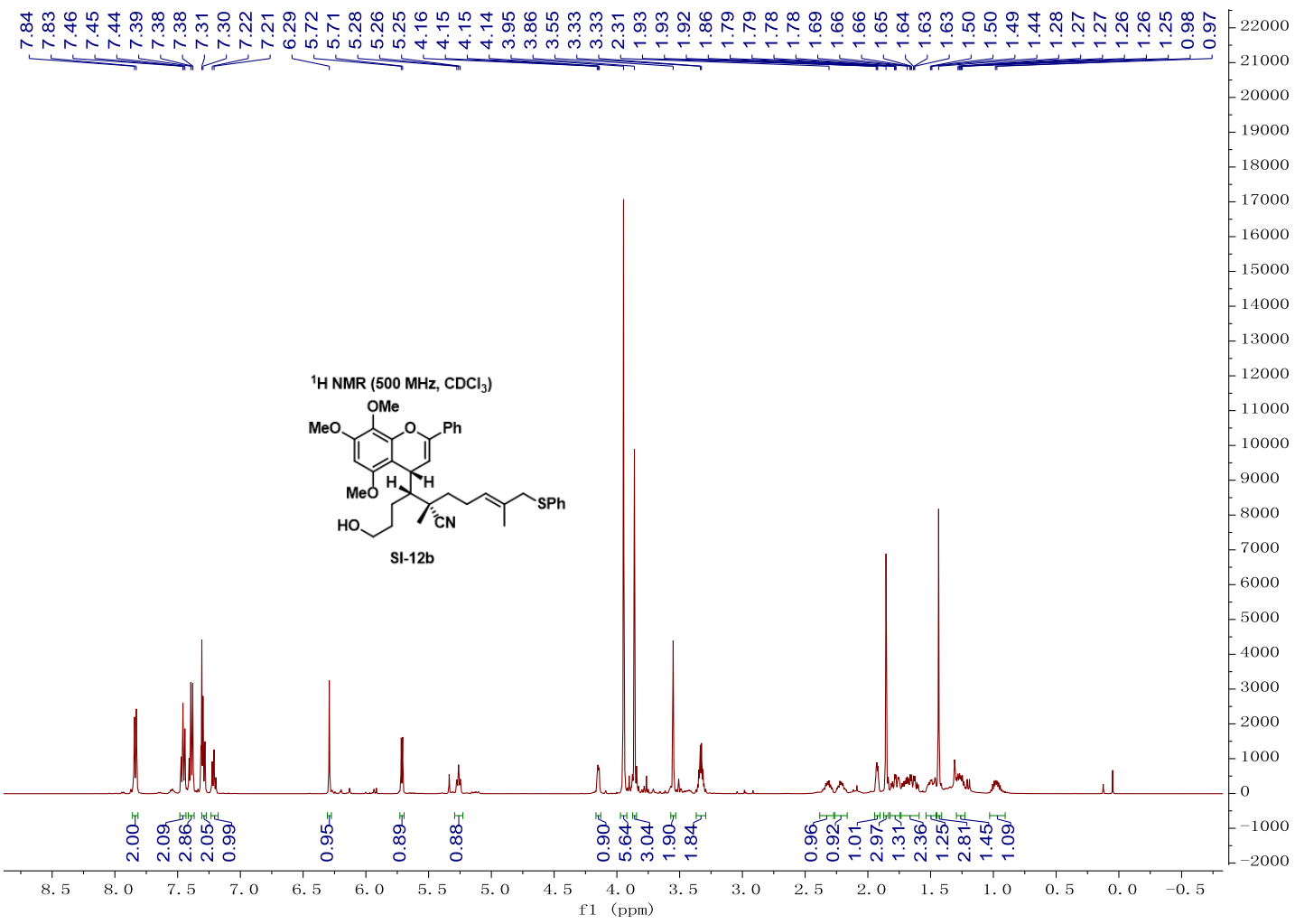

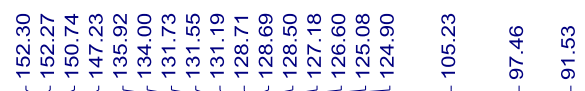

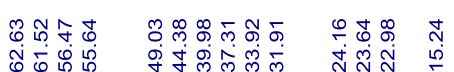

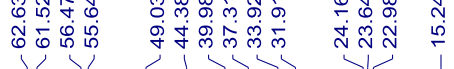

\section{$-500$}

${ }^{13} \mathrm{C} \mathrm{NMR}\left(126 \mathrm{MHz}, \mathrm{CDCl}_{3}\right)$<smiles>COCCO</smiles>

Phe

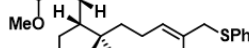

HO

SI-12b
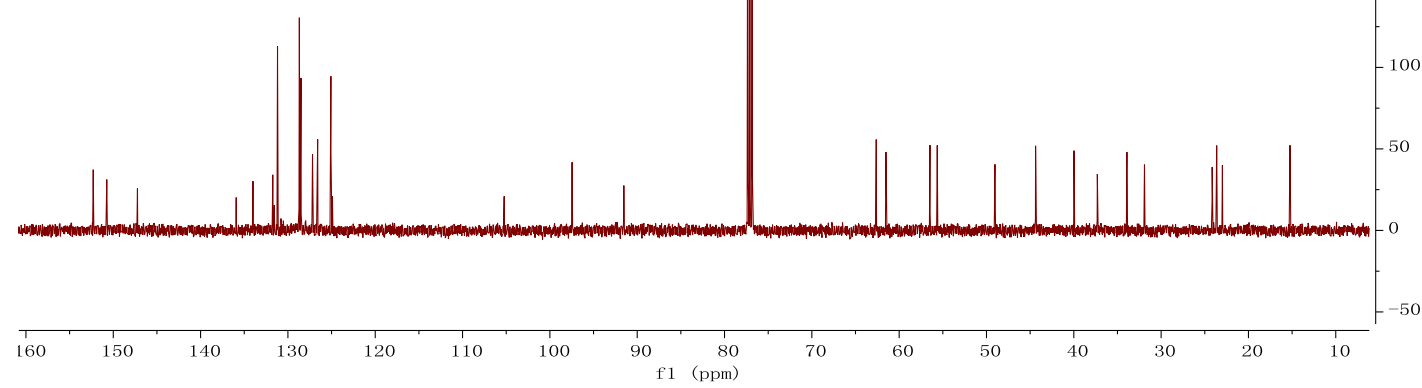

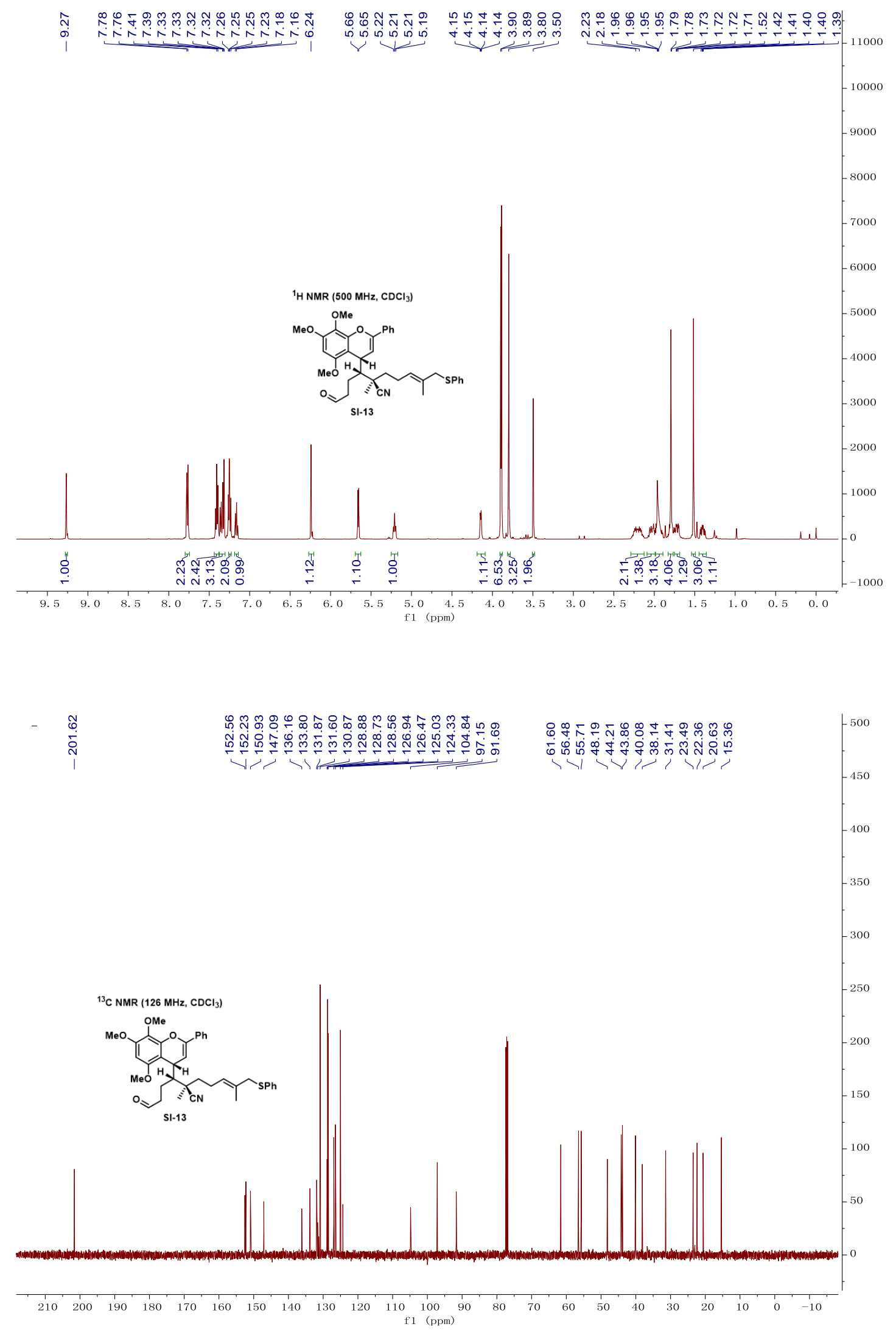


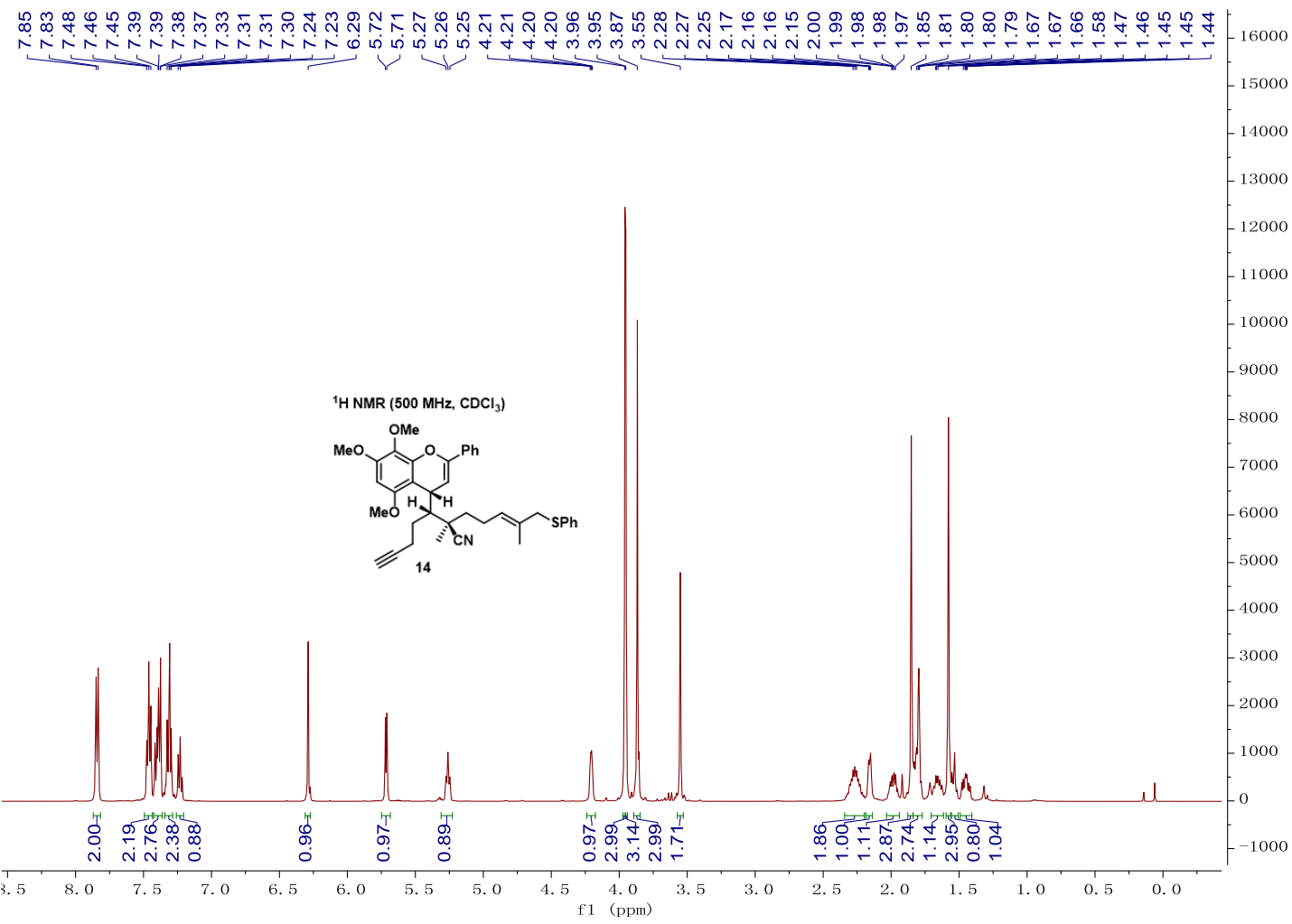

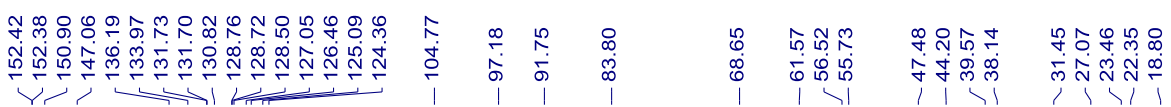

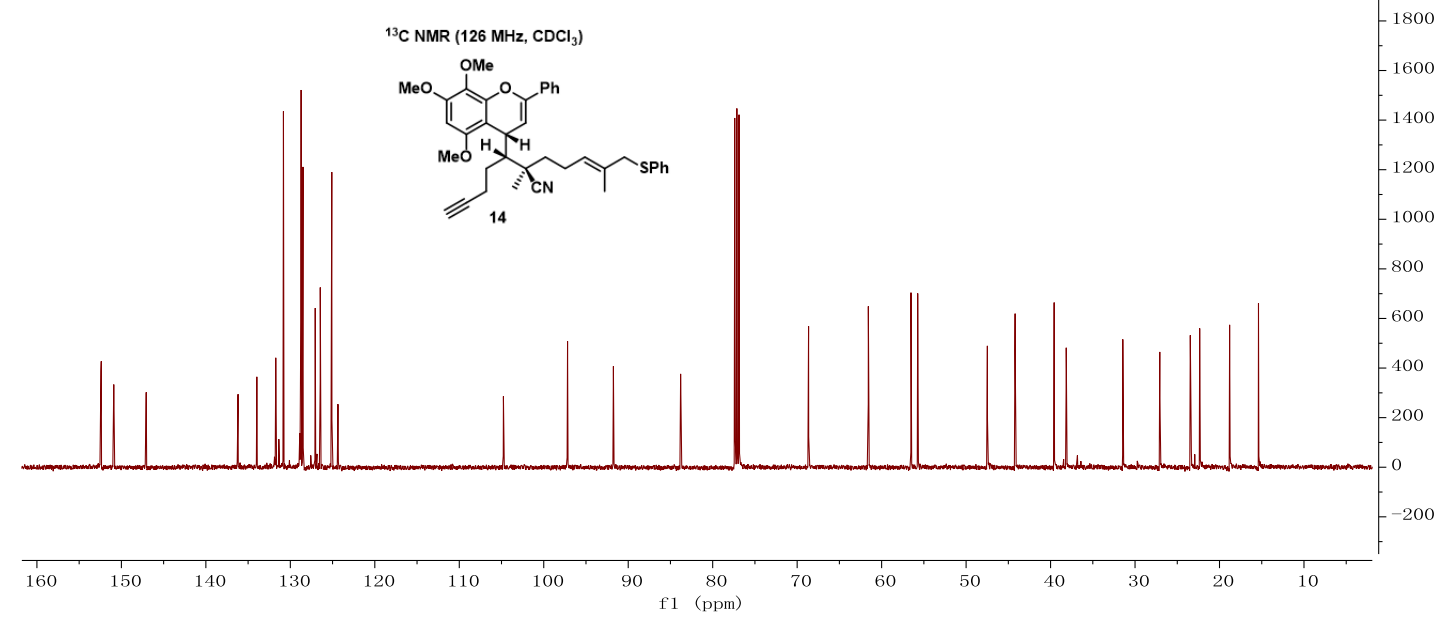


XWQ-20200929-XDY-XI-88/10 - PROTON CDC13 \{D:\2020-2\} ZHL 36
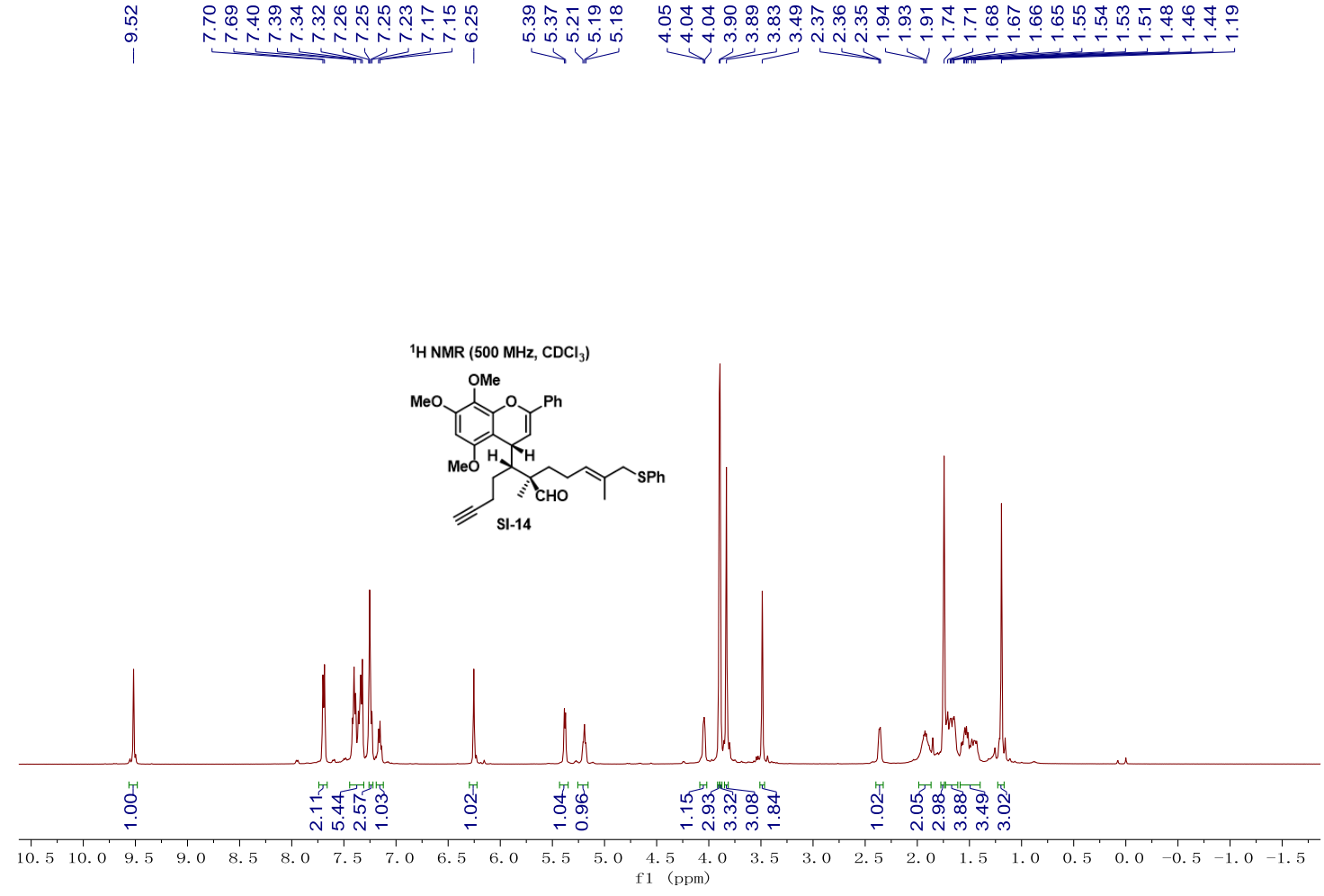

XWQ-20200929-XDY-XI-88/11 - C13CPD CDC13 $\{$ D: $\backslash 2020-2\}$ ZHL 36

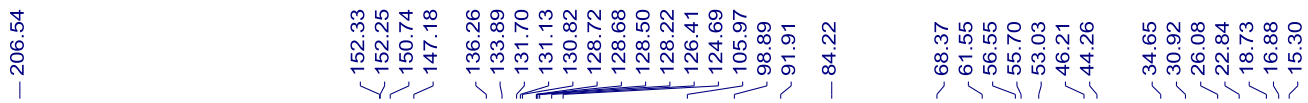

$\left.{ }^{13} \mathrm{C} \mathrm{NMR} \mathrm{(126} \mathrm{MHz,} \mathrm{CDCl}_{3}\right)$
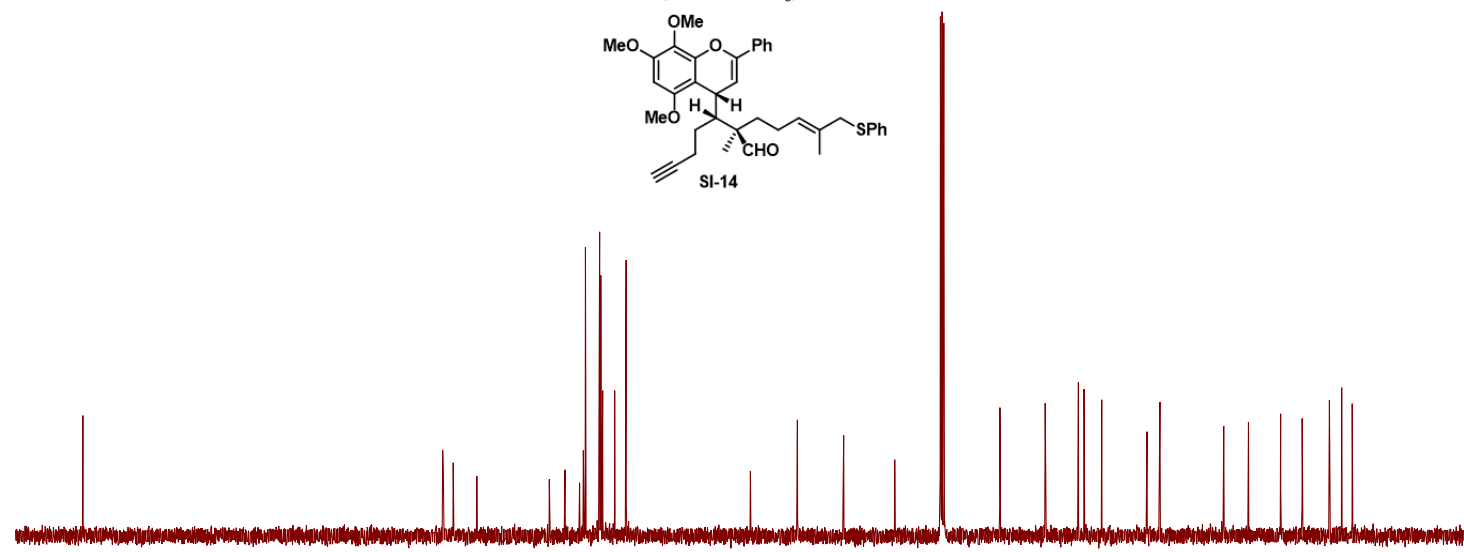

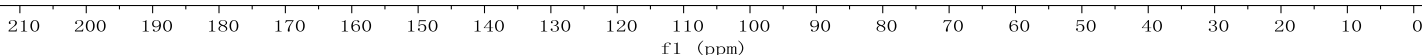




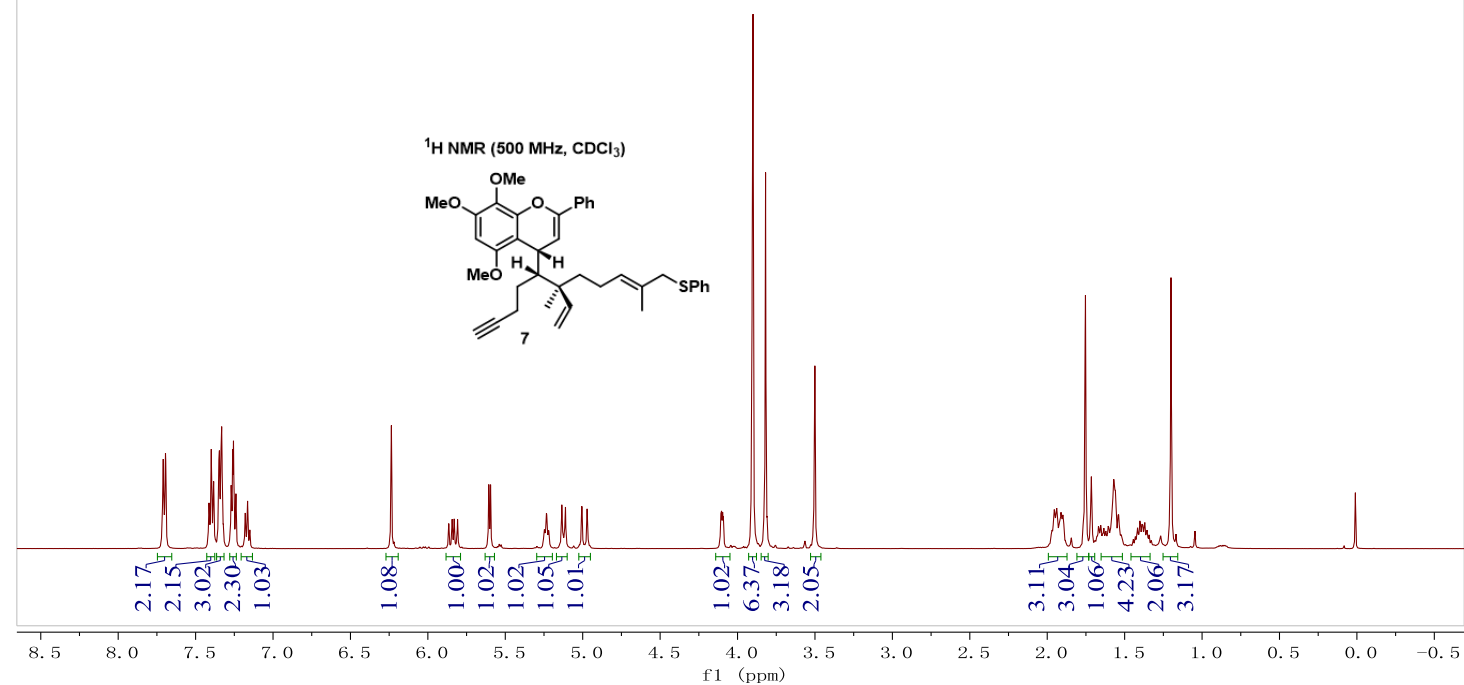

XWQ-20180828-HJD-X-69/11- C13CPD CDC13 $\{\mathrm{D}: \backslash 2018-2\}$ ZHL 29

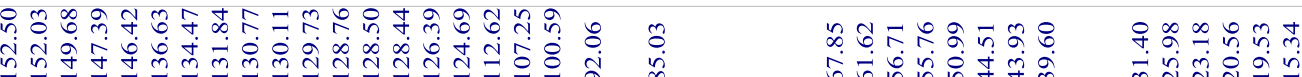

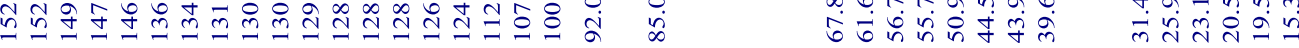

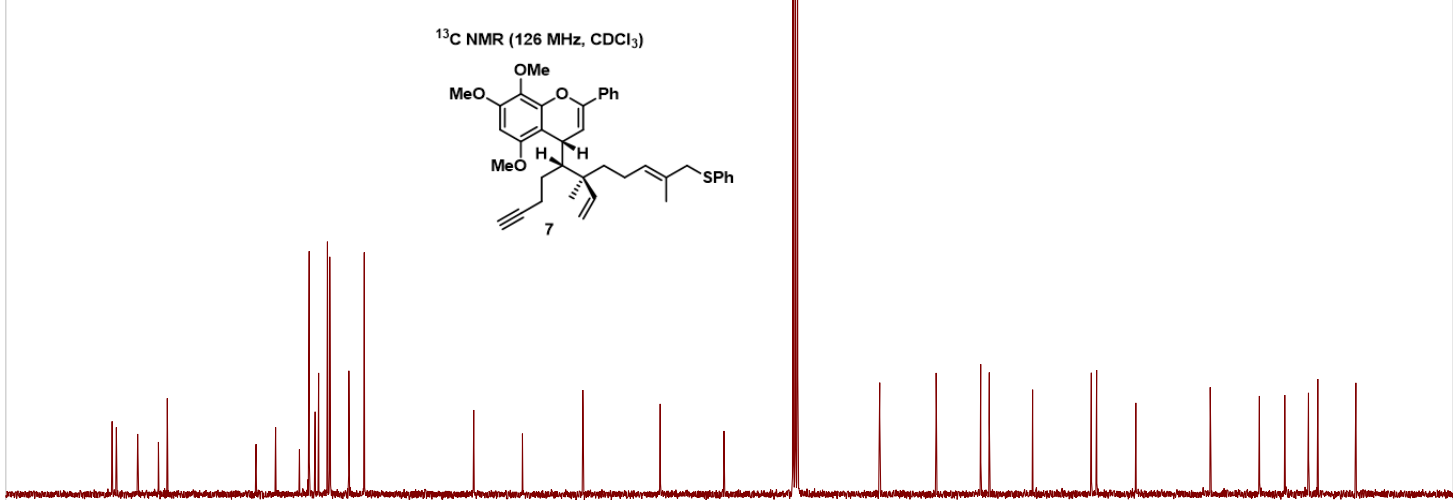

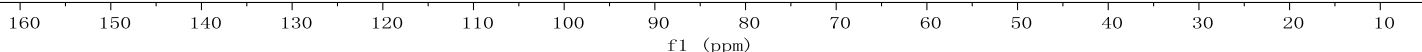



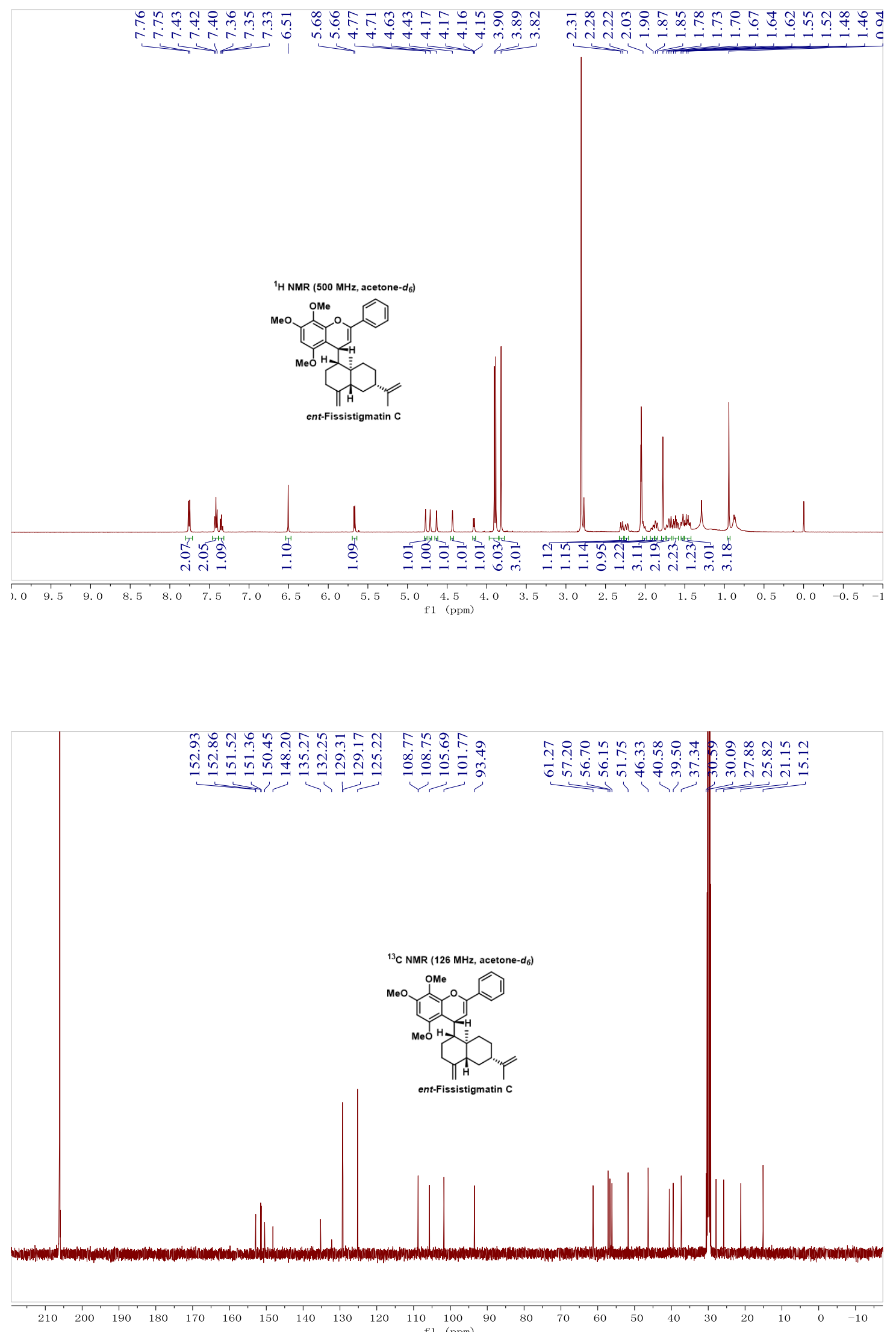
XWQ-20181210-HJD-XI-26-1/10 - PROTON CDC13 $\{$ D: \2018-3\} ZHL 46

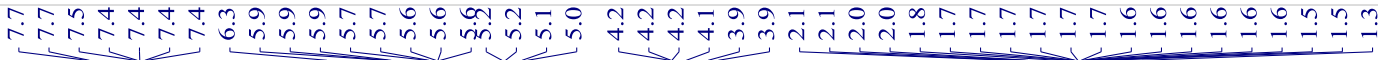
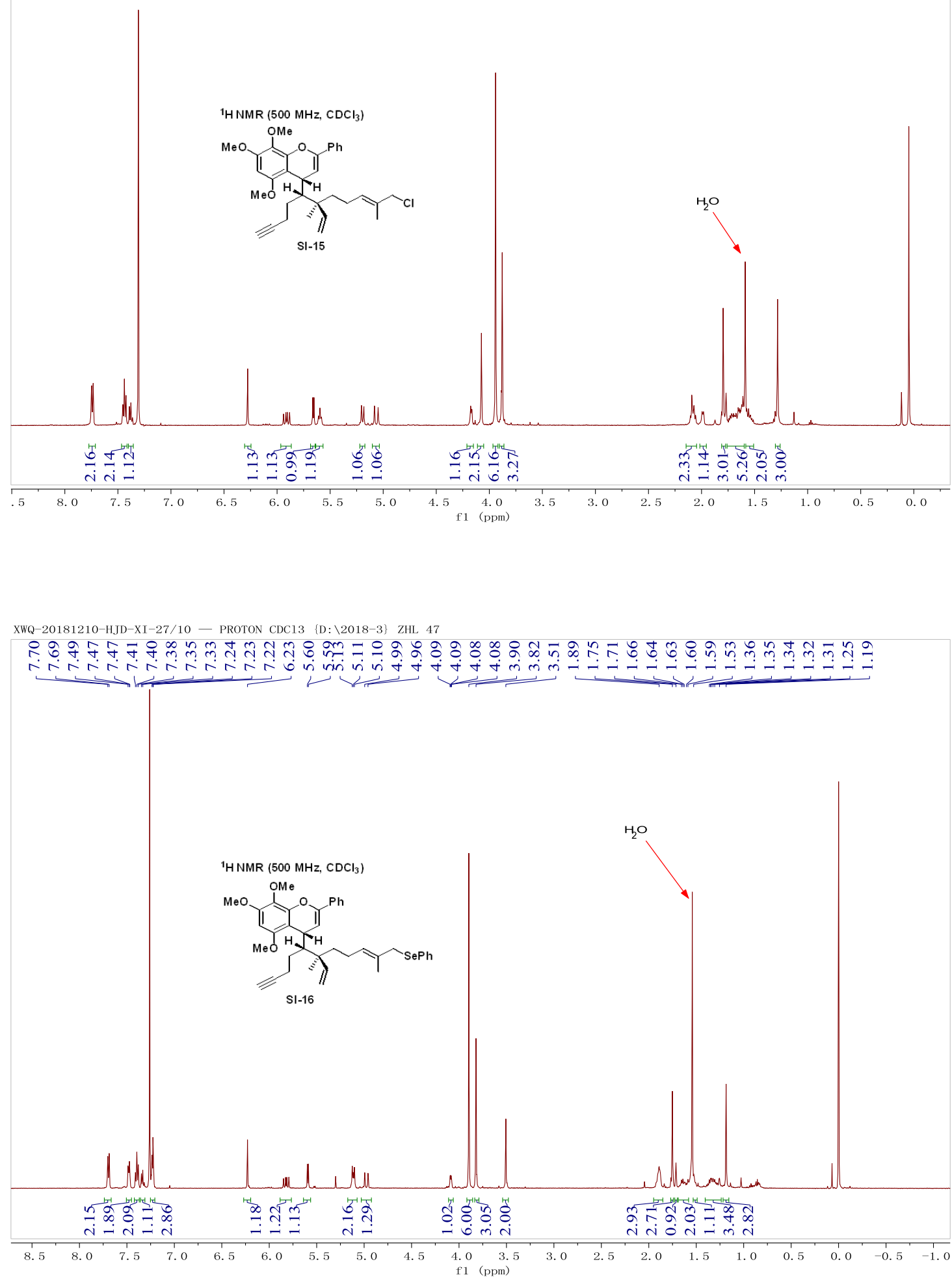

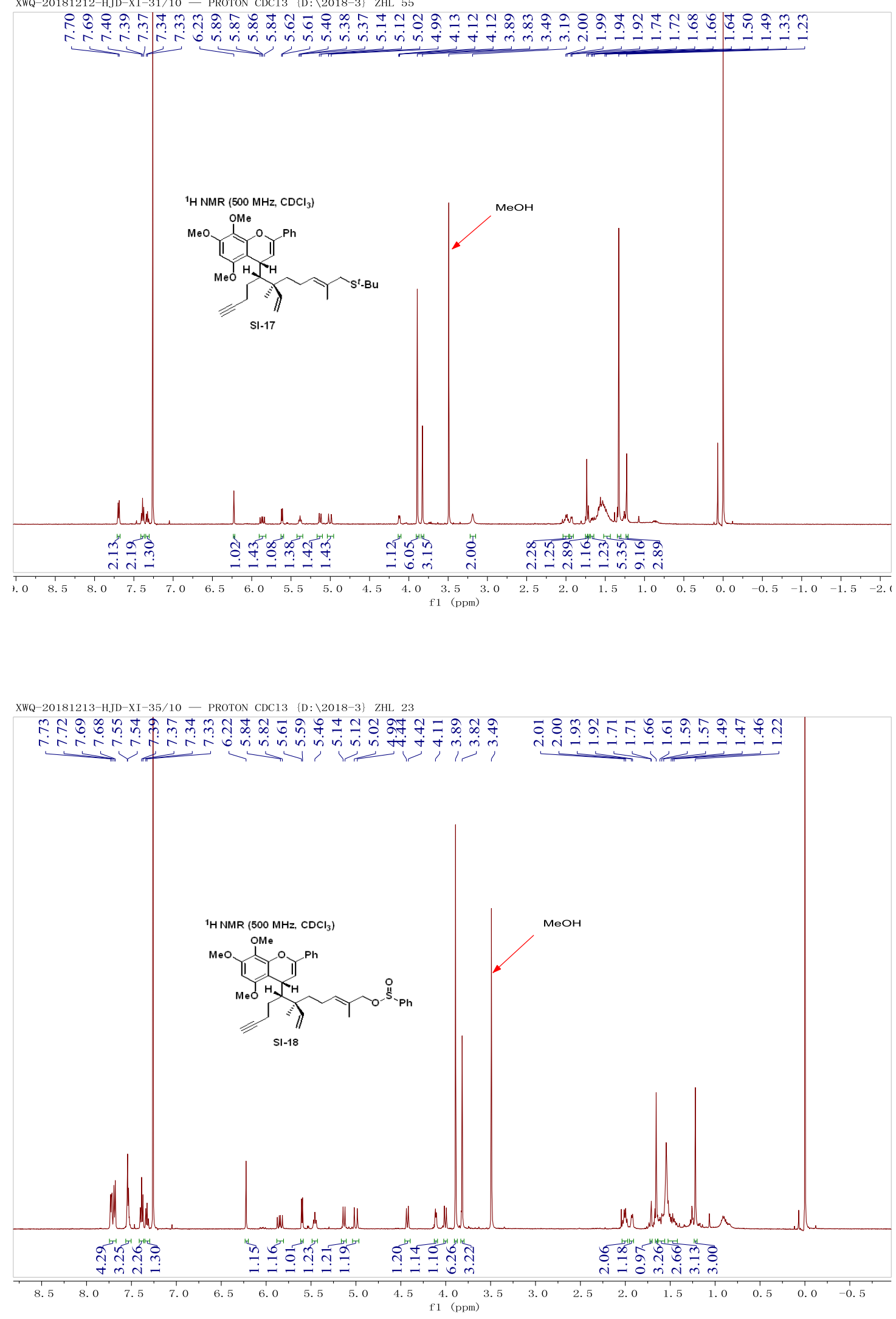
XWQ-20181213-HJD-XI-34/10 - PROTON CDC13 \{D: \2018-3\} ZHL 22

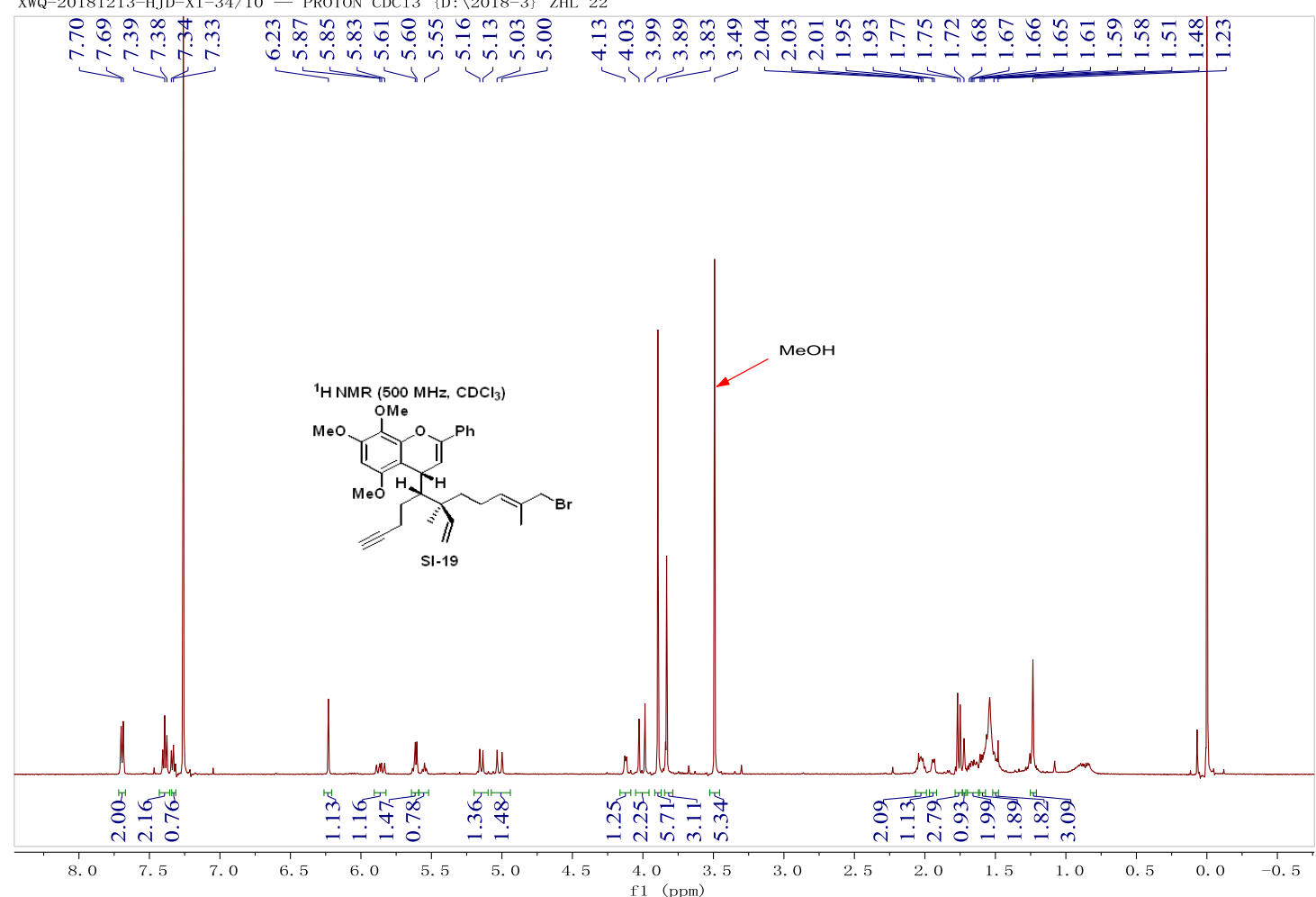




\section{HRMS data for compounds}
National Center for Organic Mass Spectrometry in Shanghai Shanghai Institute of Organic Chemistry
Chinese Academic of Sciences

High Resolution MS DATA REPORT

Instrument: Thermo Fisher Scientific LTQ FTICR-MS

Card Serial Number : D182688

Sample Serial Number: NO.12 HJD-VII-39

Operator : DONG Date: $2018 / 09 / 10$

Operation Mode: ESI POSITIVE Ion Mode

Elemental composition search on mass 511.25

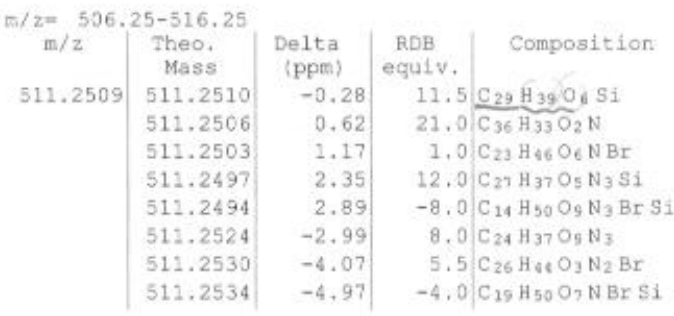<smiles>CCCC[C@@](C=O)(CCCOC(C)(C)C)C1=CC(c2ccccc2)Oc2c(OC)c(OC)cc(OC)c21</smiles>

(BBS 


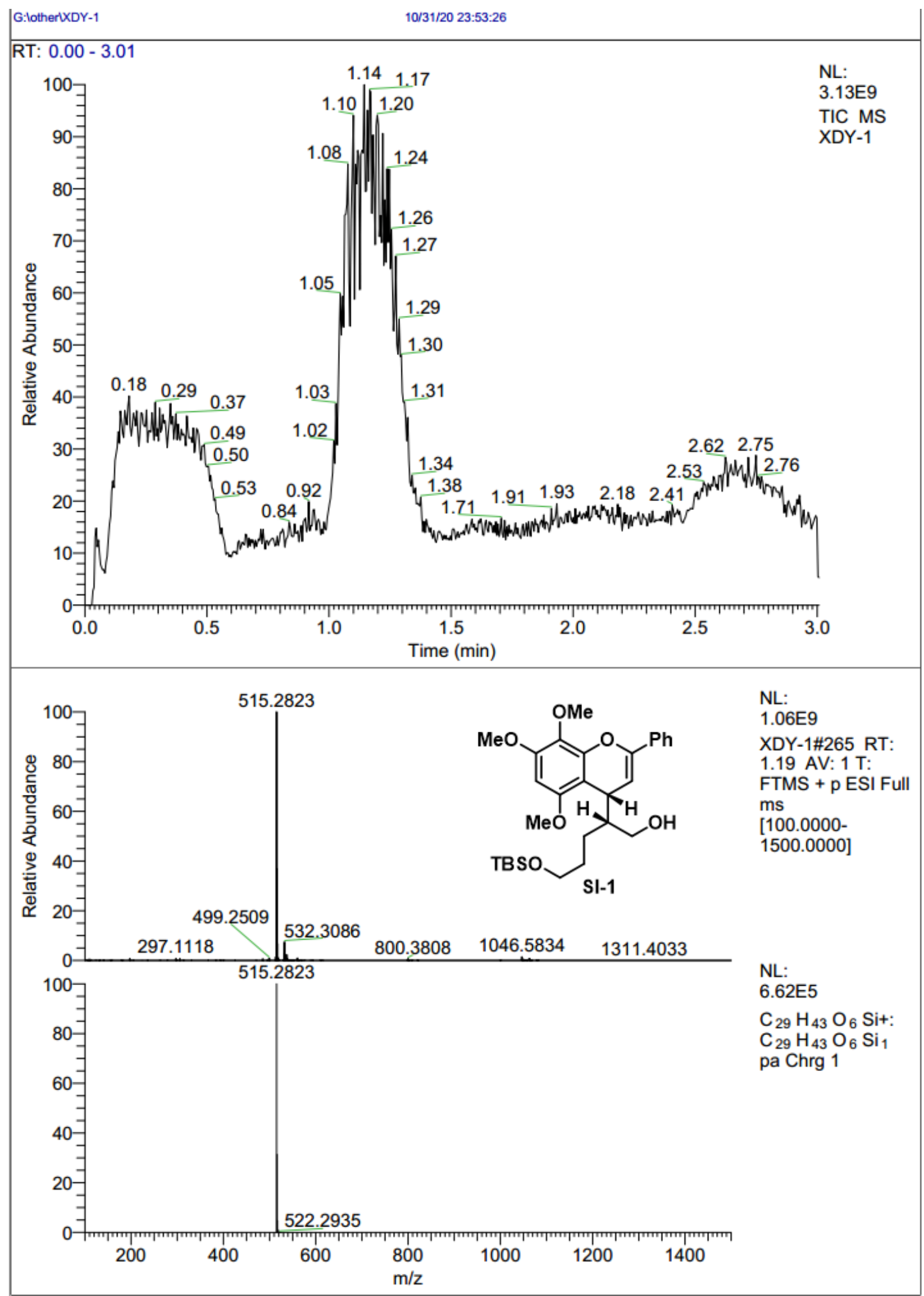




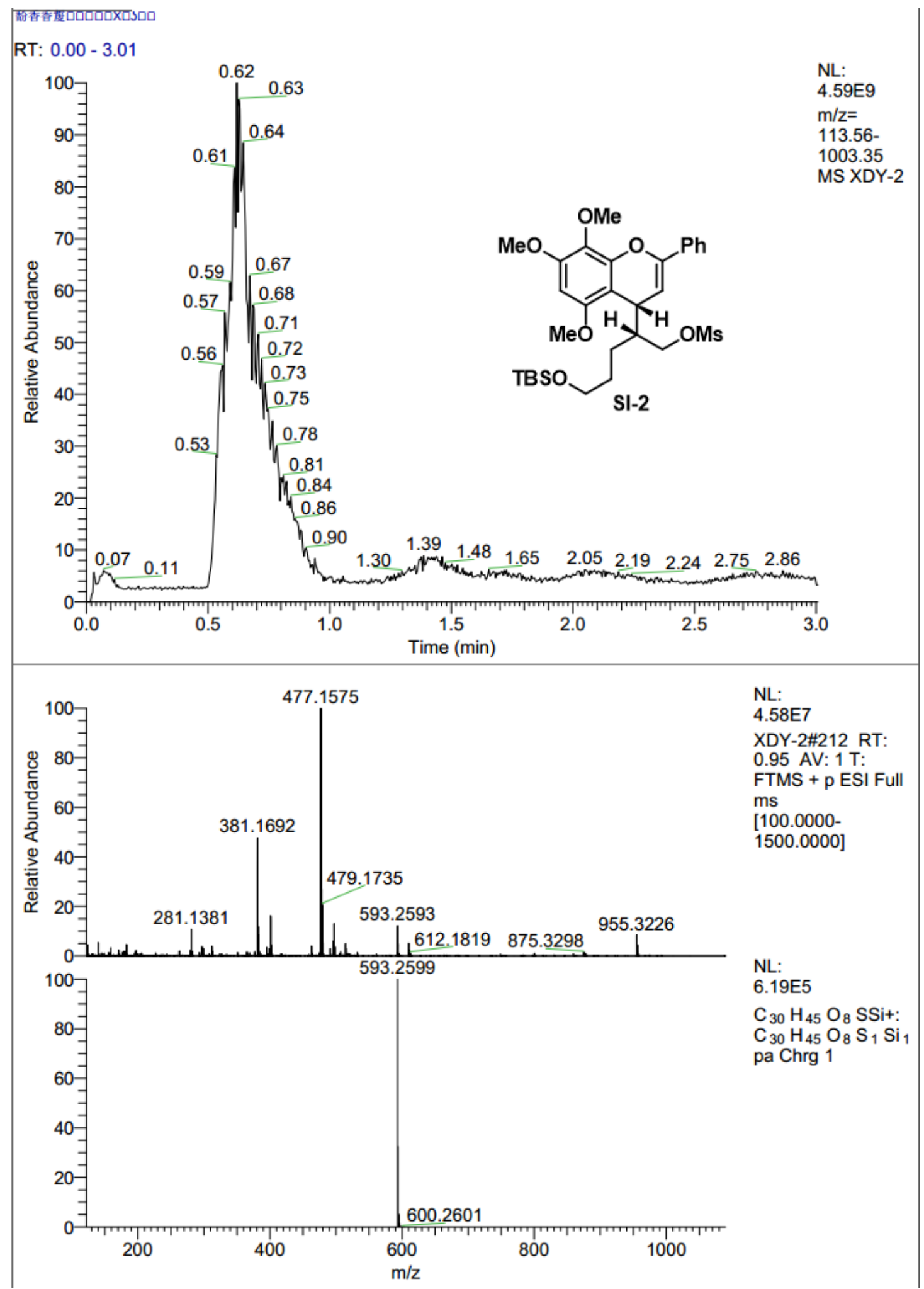




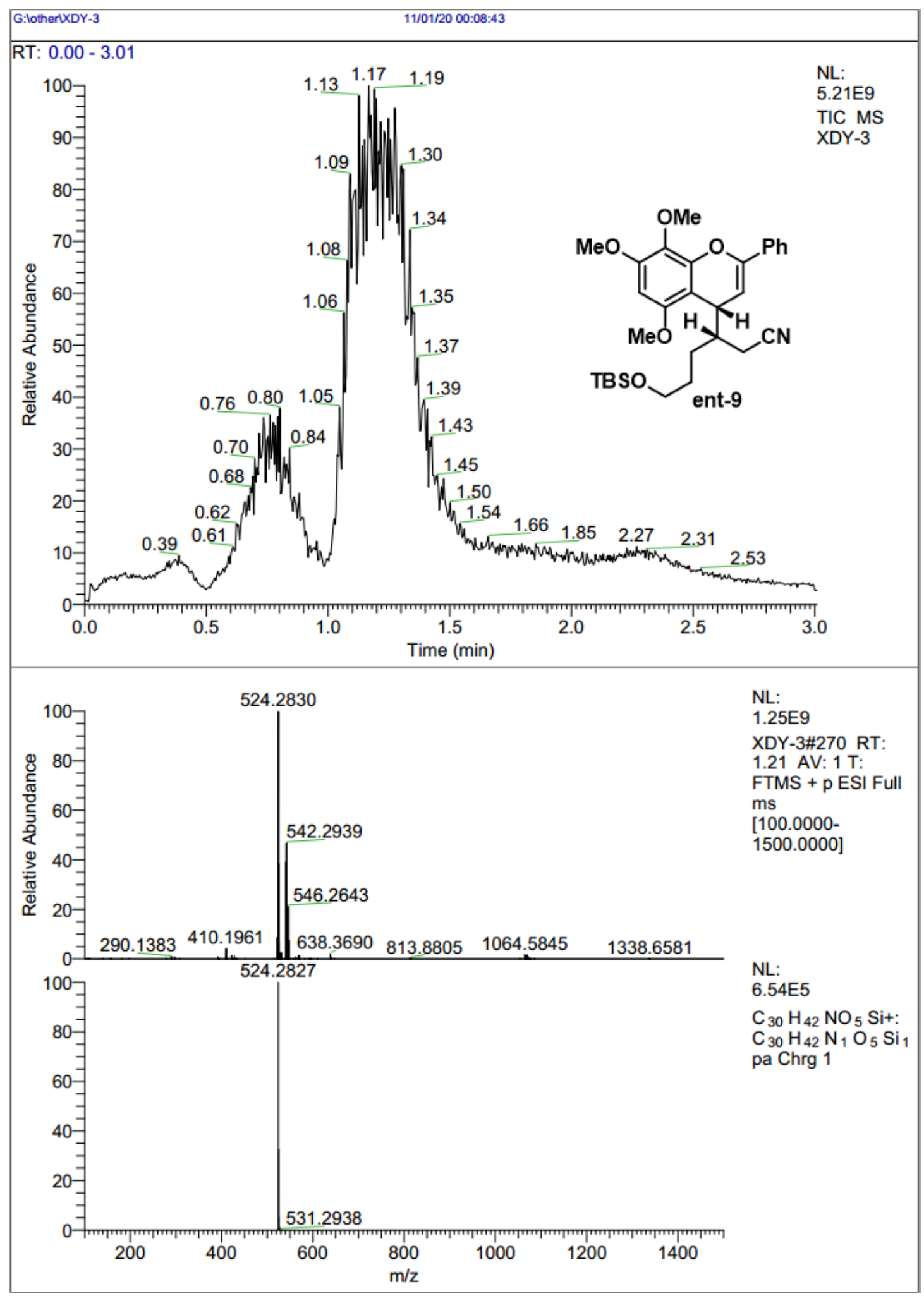




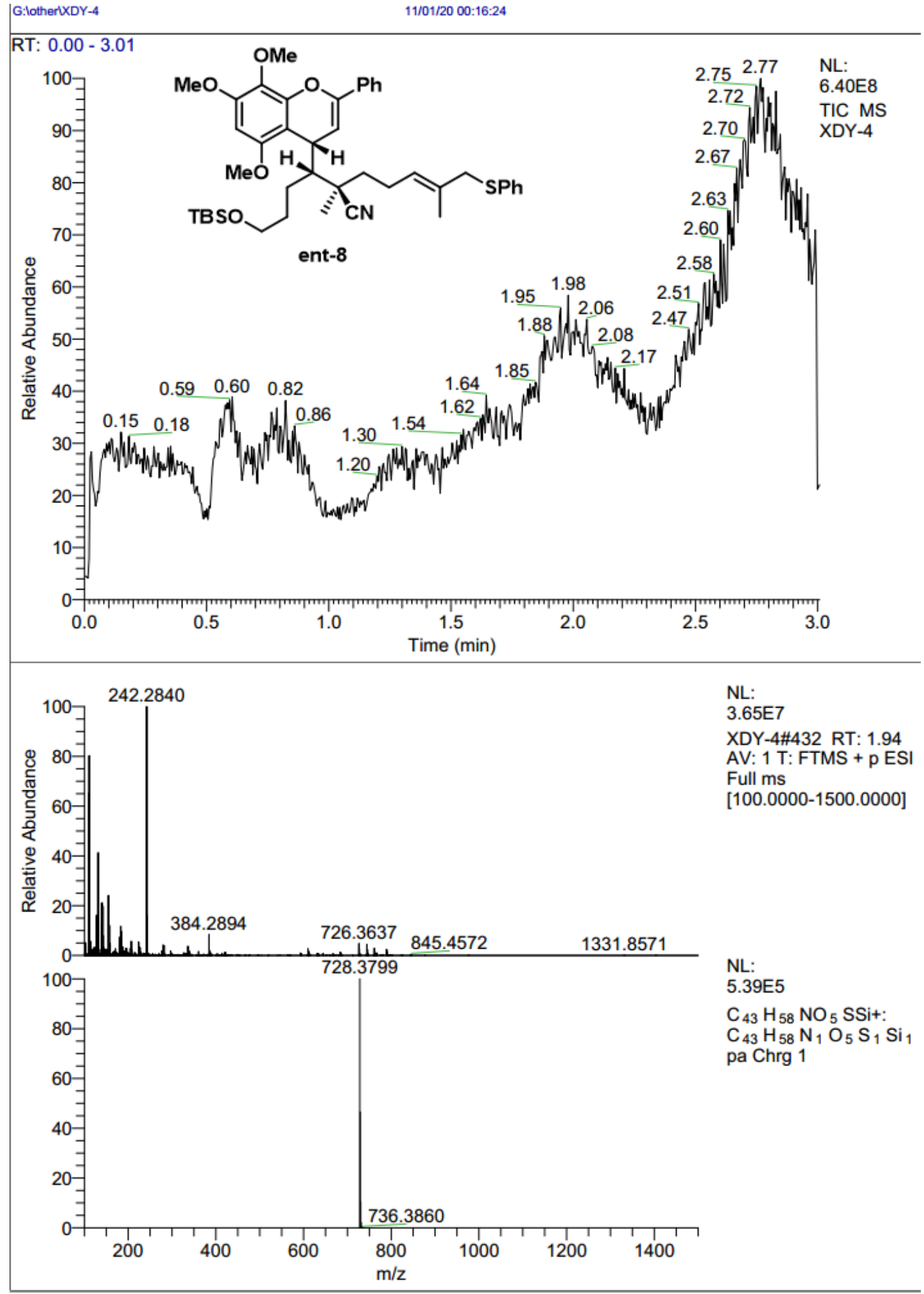




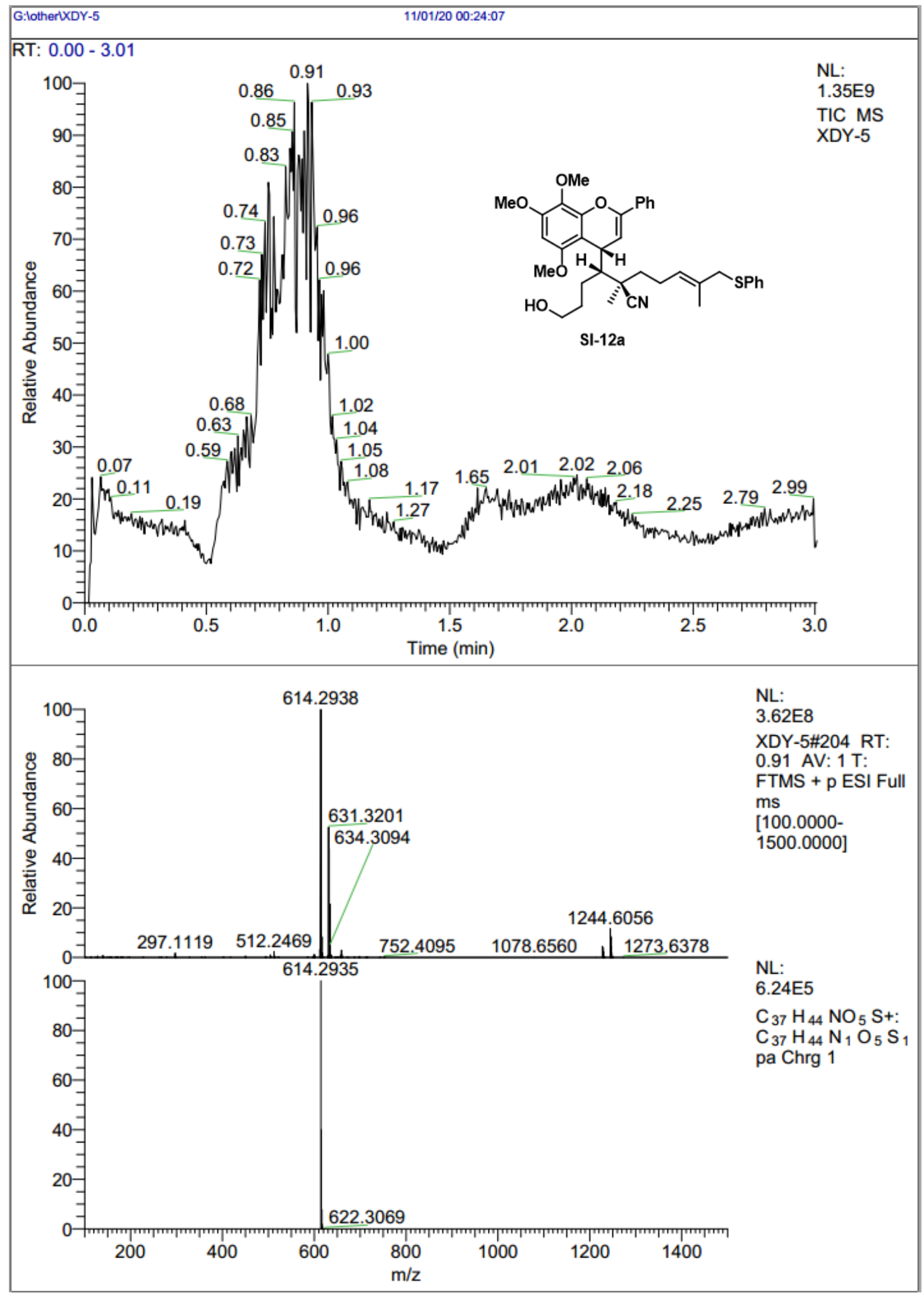




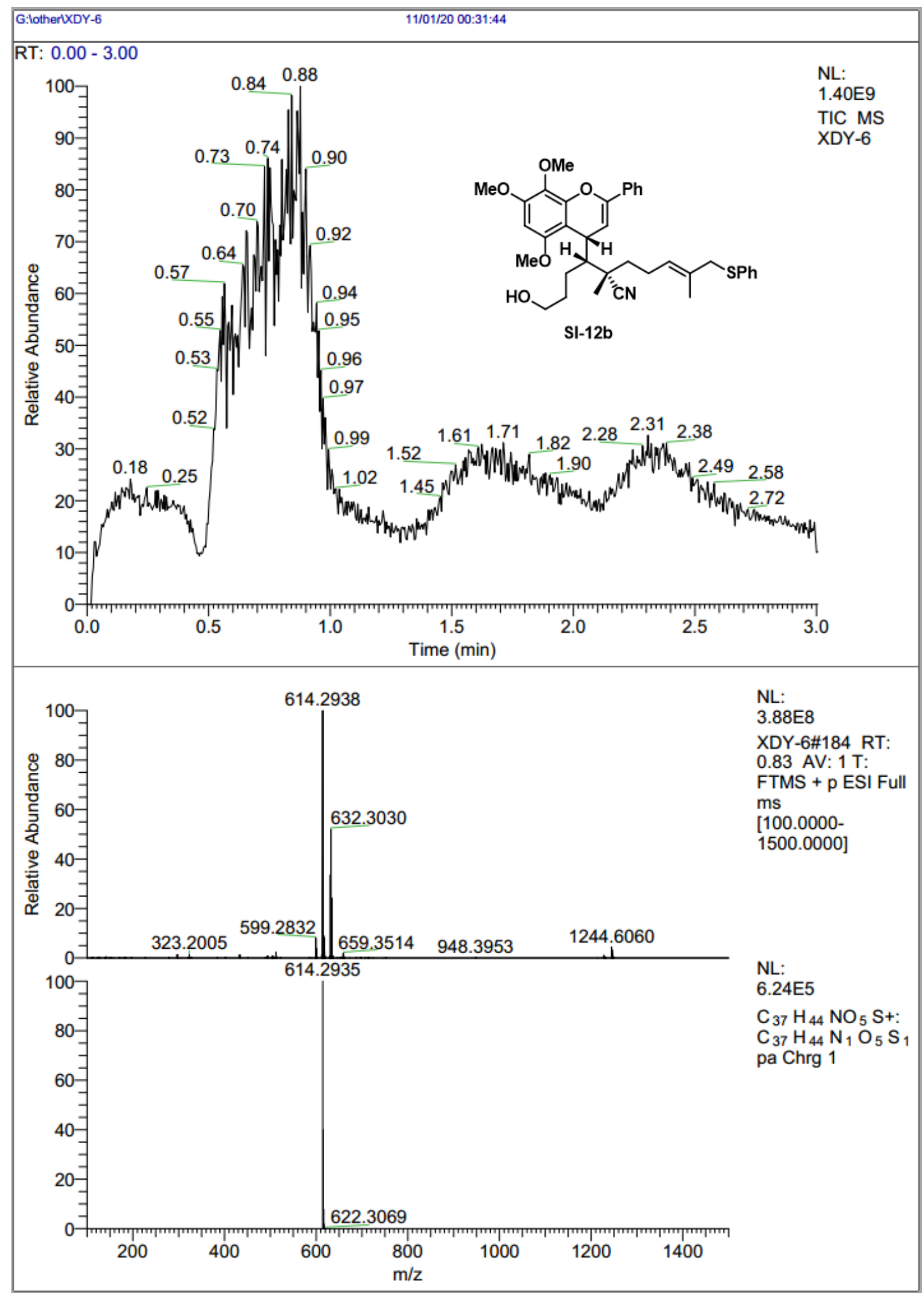




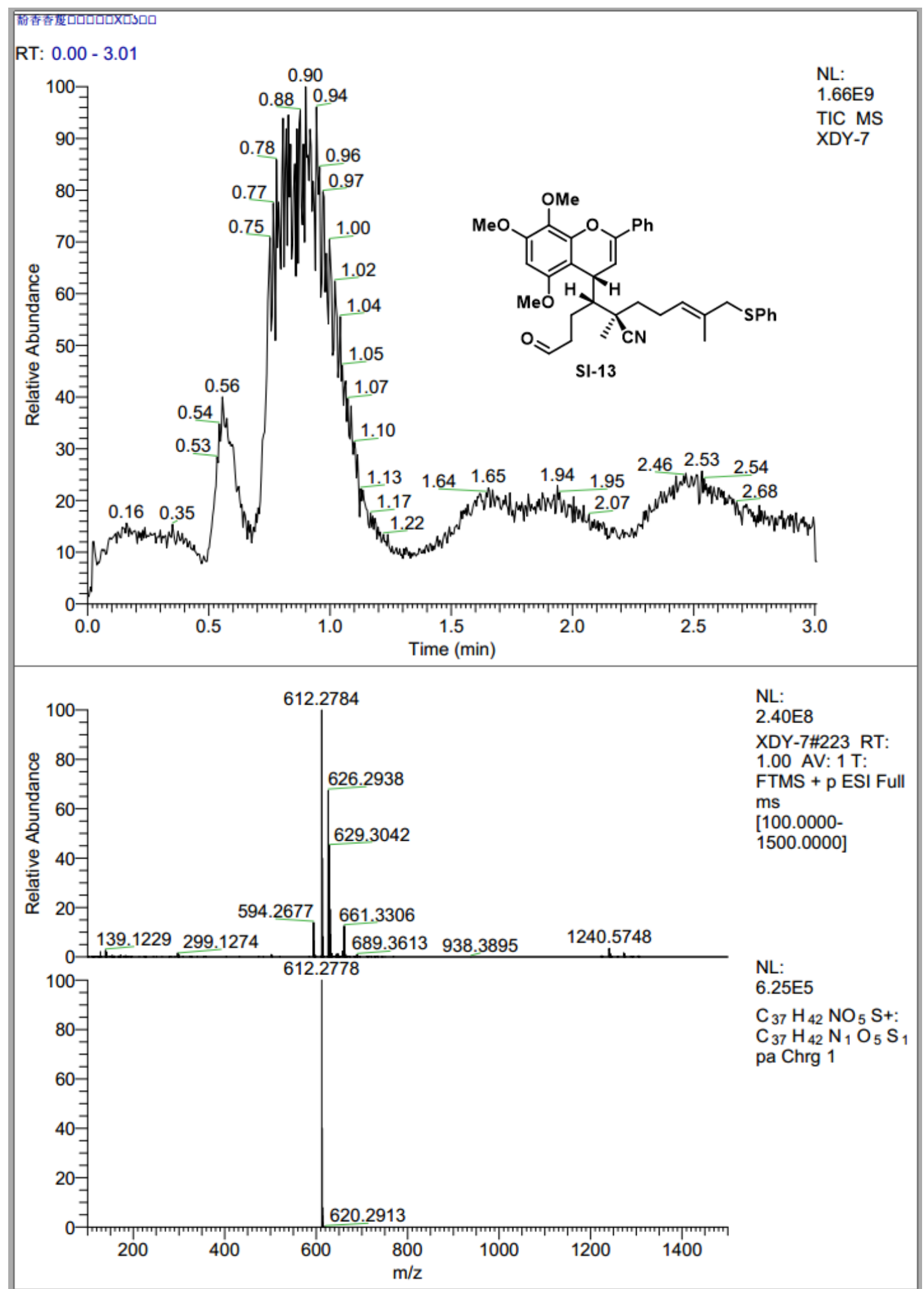




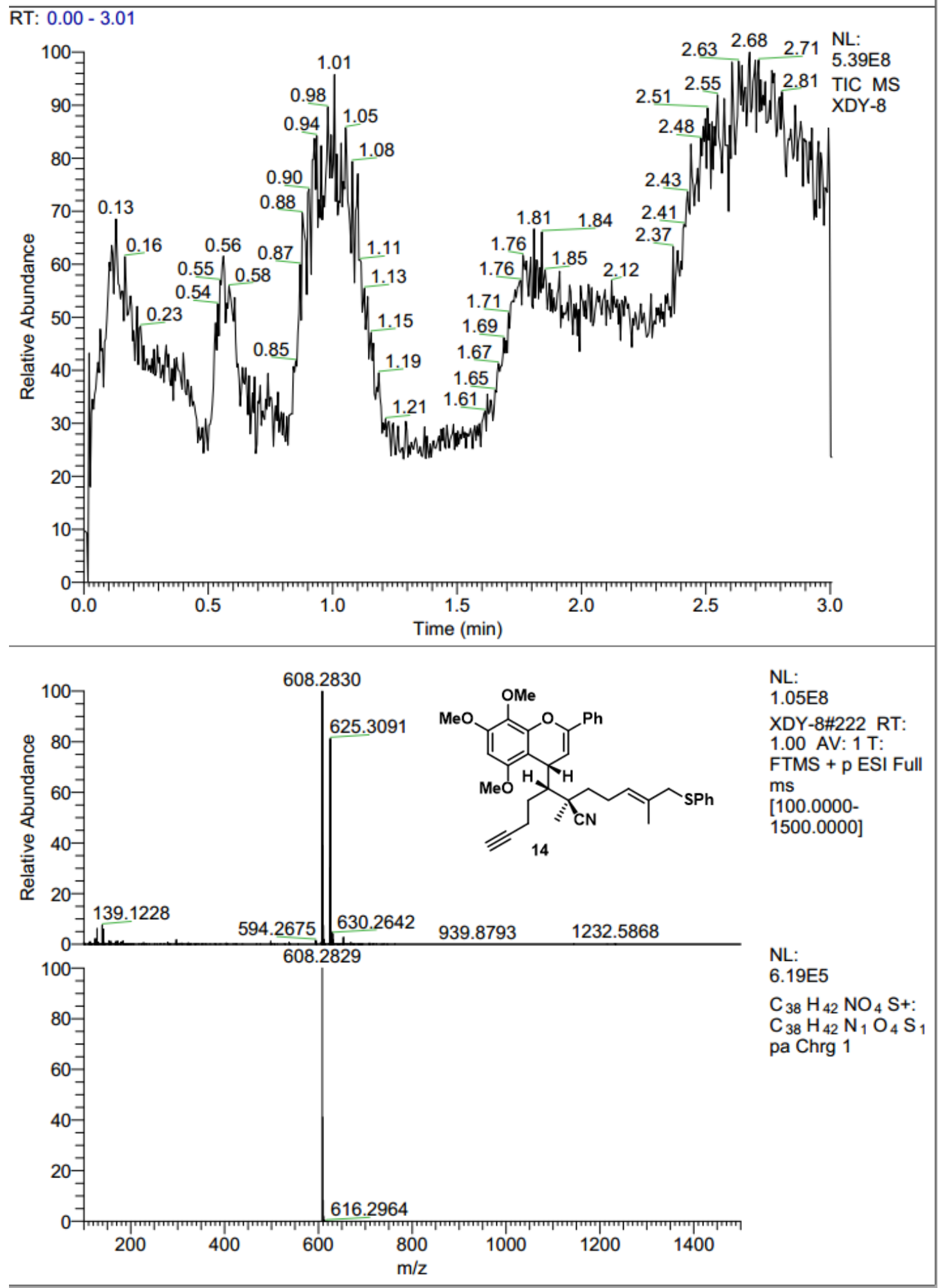




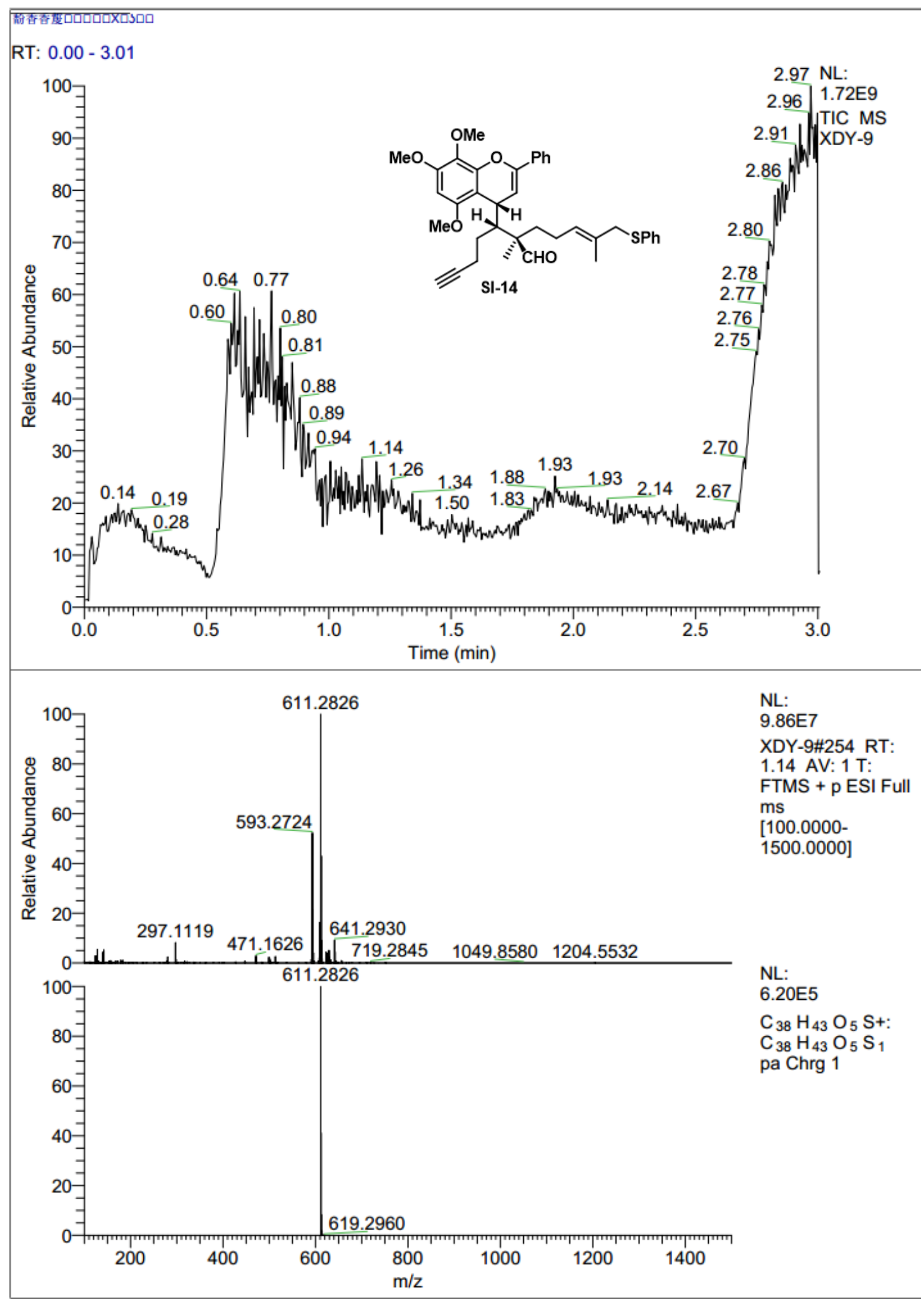




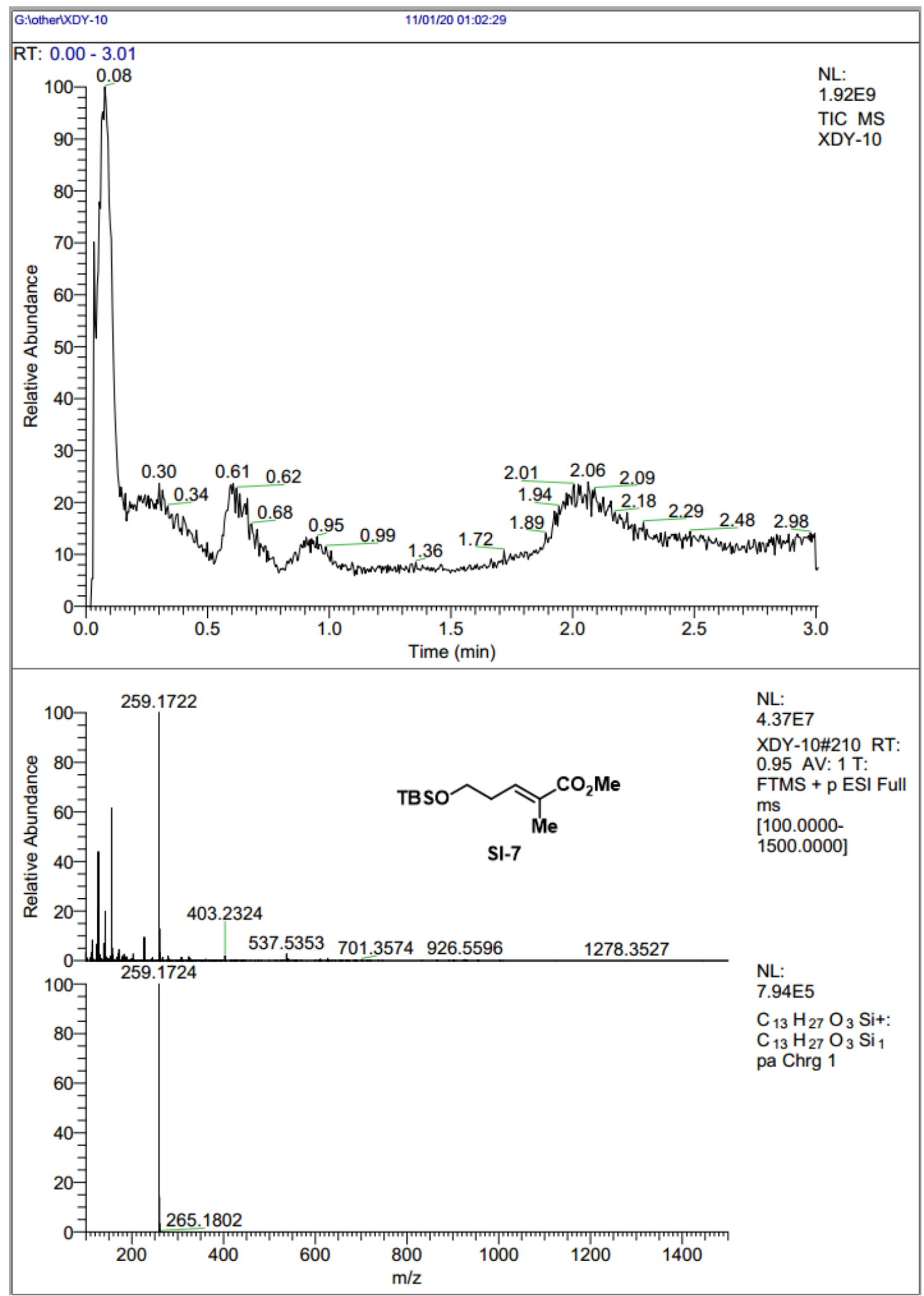




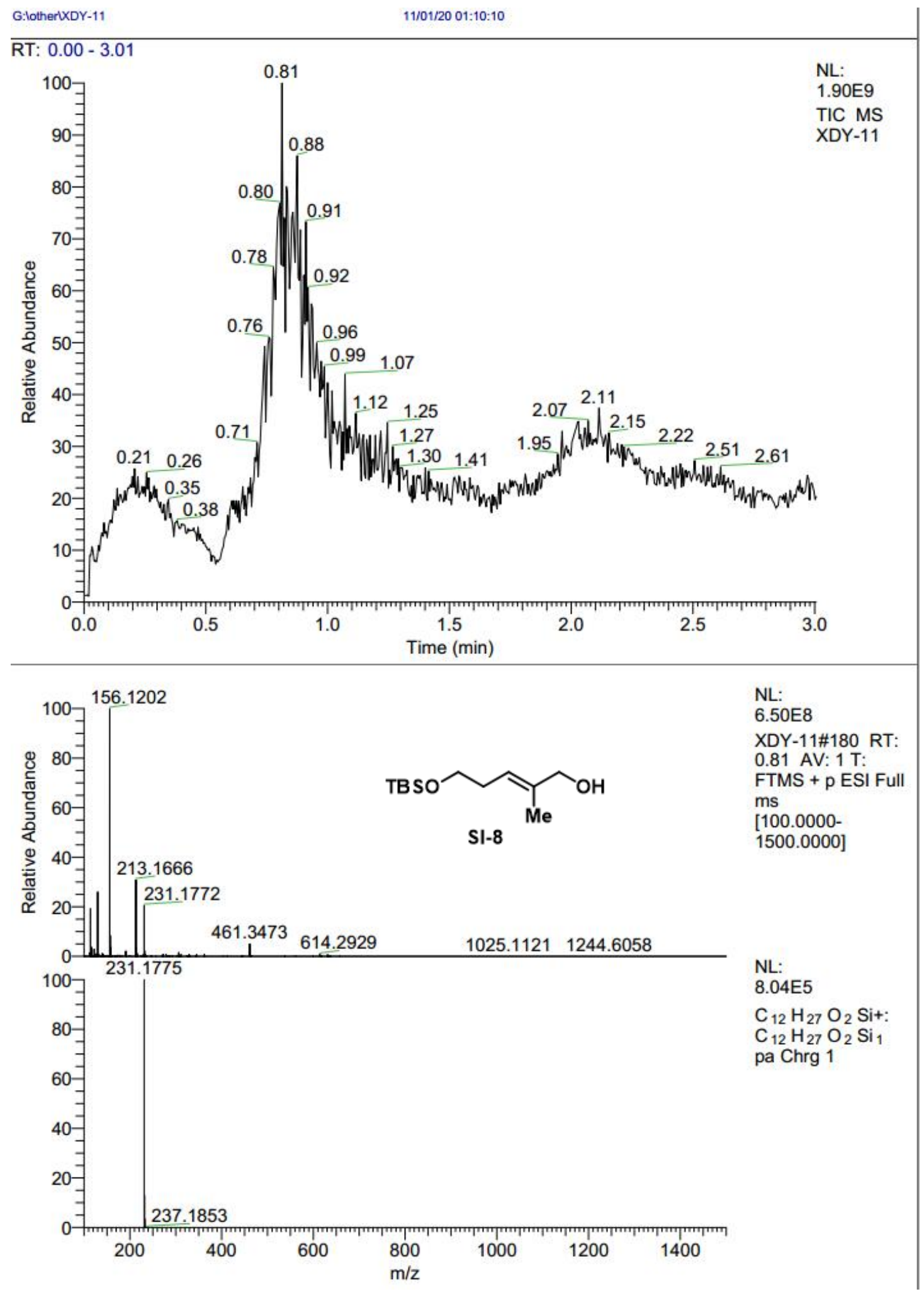




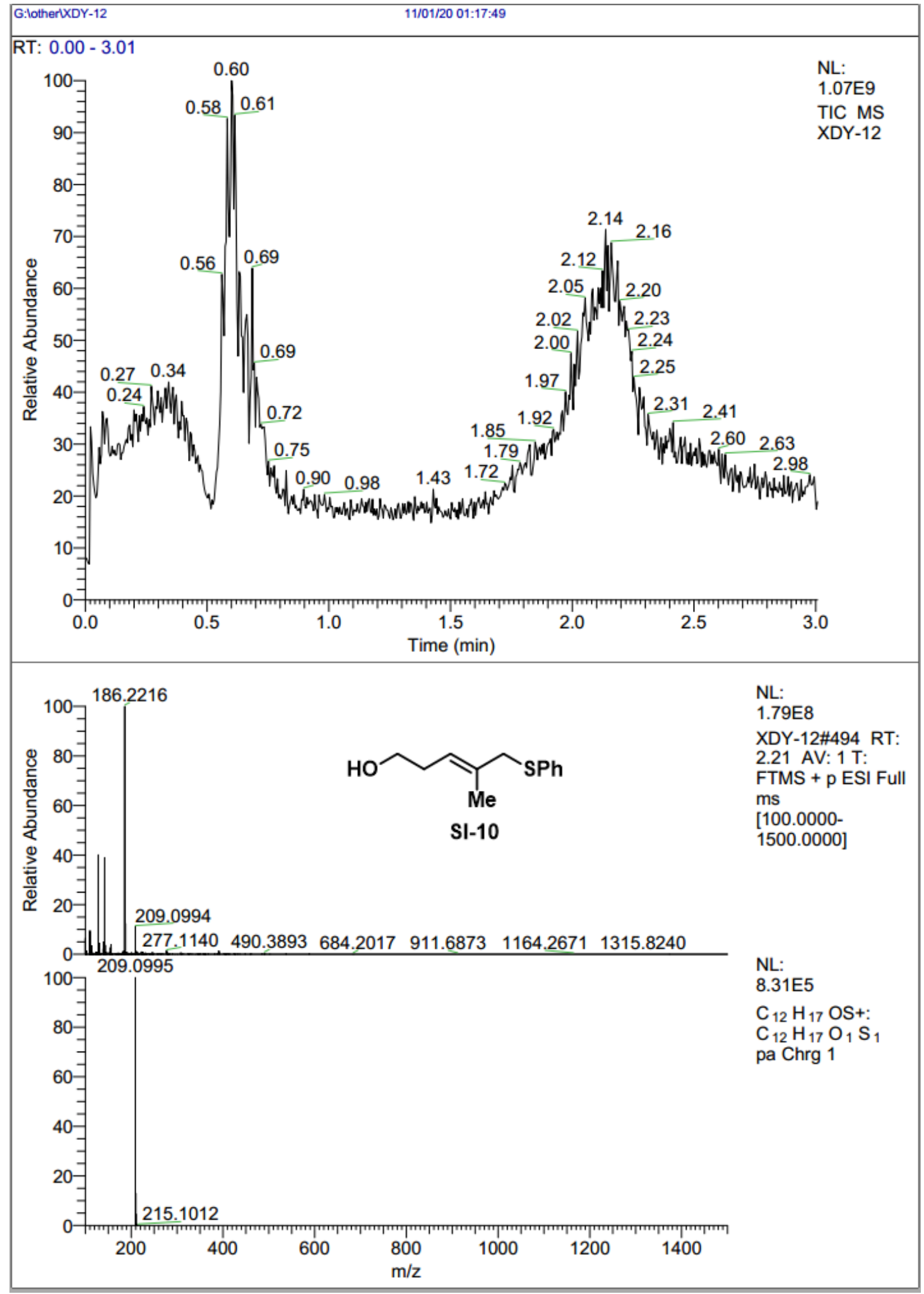




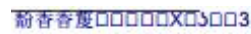

RT: $0.00-3.01$
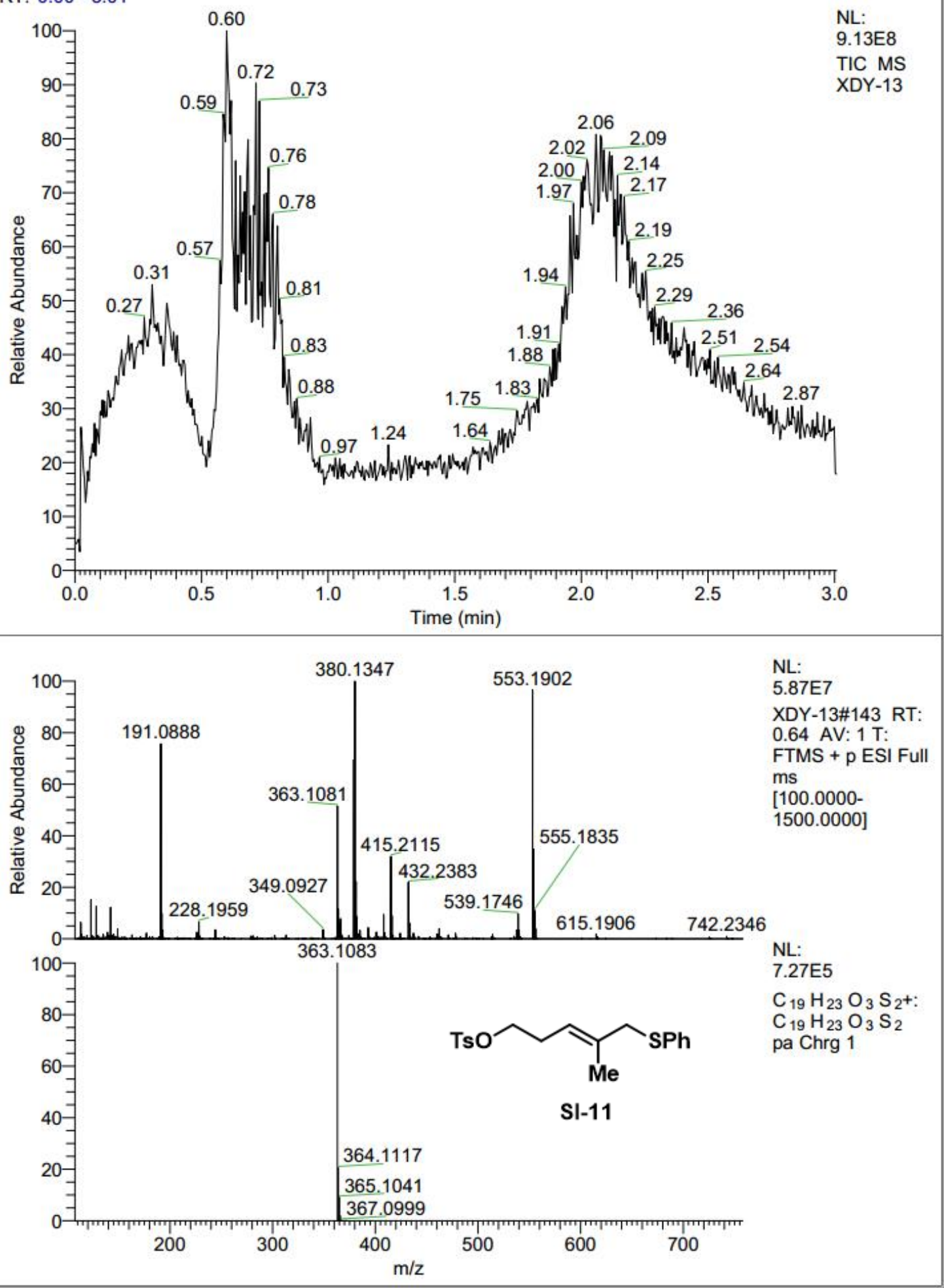


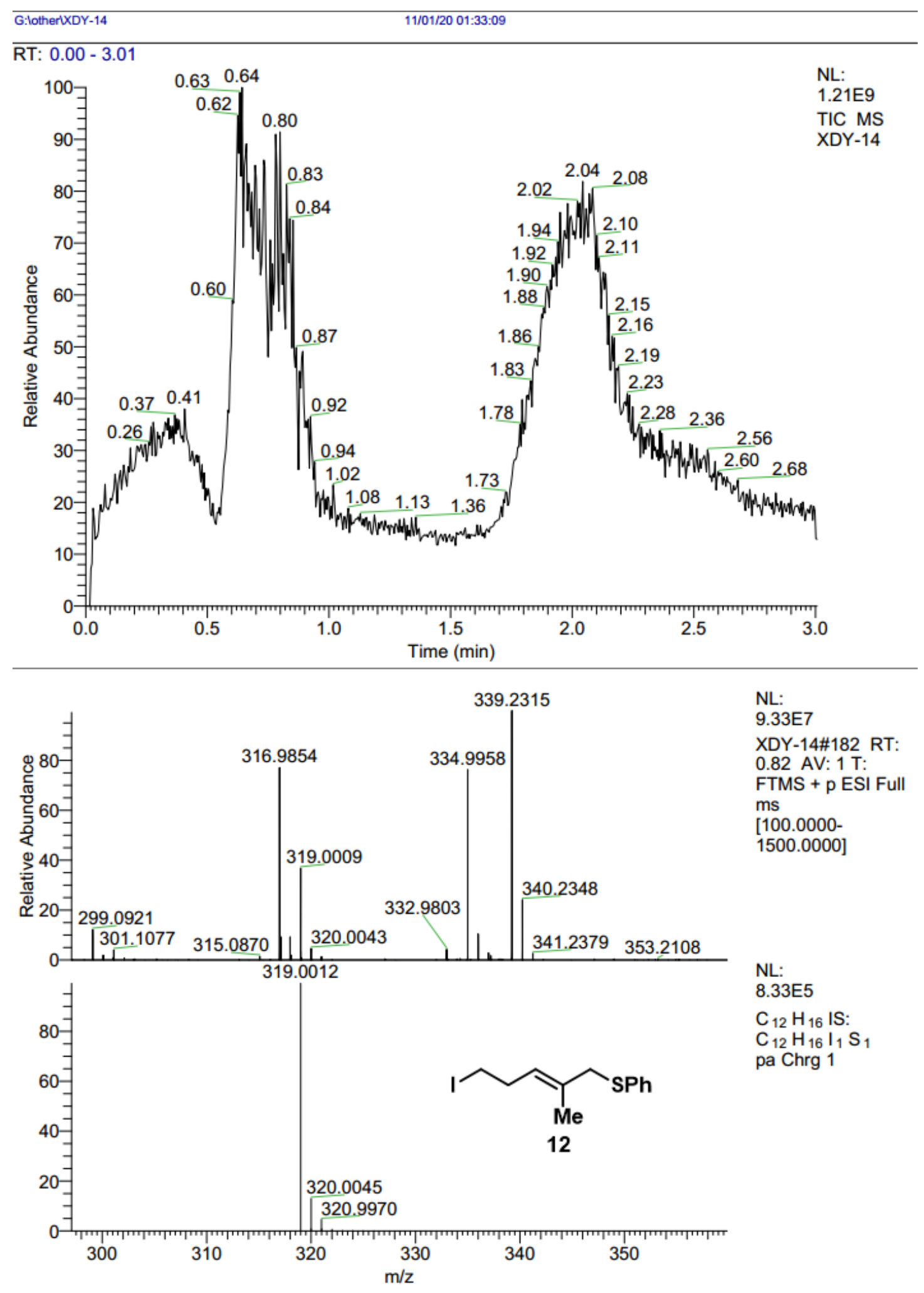


National Center for Organic Mass Spectrometry in Shanghai Shanghai Institute of Organic Chemistry

Chinese Academic of Sciences

High Resolution MS DATA REPORT

Instrument: Thermo Fisher Scientific LTQ FTICR-MS

Card Serial Number : D182588

Sample Serial Number: NO.33 HJD-X-69

Operator : DONG

Date: $2018 / 09 / 04$

Operation Mode: DART POSITIVE Ion Mode

Elemental composition search on mass 609.30

$\mathrm{m} / \mathrm{z}=604 \cdot 30-614 \cdot 30$

\begin{tabular}{l|l|l|l|l}
$\mathrm{m} / \mathrm{z}$ & Theo. & Delta & RDB & Composition
\end{tabular}

\begin{tabular}{l|r|r|r}
\multicolumn{1}{c|}{$\begin{array}{c}\text { Mass } \\
609.3 \mathrm{C} 20\end{array}$} & [Ppm] & equiv. & \\
6.53 & 18.0 & $\mathrm{C}_{37} \mathrm{H}_{43} \mathrm{O}_{3} \mathrm{~N}_{3} \mathrm{~S}$
\end{tabular}

$609.3033 \quad-1.67 \quad 17.5 \mathrm{C}_{39} \mathrm{H}_{45} \mathrm{O}_{4} \mathrm{~S}$

$6 0 9 . 3 0 0 6 \quad 2 . 7 4 \quad 1 8 . 5 \longdiv { \mathrm { C } _ { 3 5 } \mathrm { H } _ { 4 2 \mathrm { O } _ { 2 } \mathrm { N } _ { 6 } \mathrm { S } } }$

\begin{tabular}{l|l|l|l|l|}
609.3045 & -3.56 & 19.5 & $\mathrm{C}_{32} \mathrm{H}_{37} \mathrm{O}_{3} \mathrm{~N}_{10}$
\end{tabular}

$609.3046 \quad-3.86 \quad 22.5 \mathrm{C}_{40 \mathrm{H}_{41} \mathrm{~N}_{4} \mathrm{~S}}$

\begin{tabular}{ll|l|l|l}
609.2993 & 4.94 & 19.0 & $\mathrm{C}_{33} \mathrm{H}_{39} \mathrm{ON} 9 \mathrm{~S}$
\end{tabular}

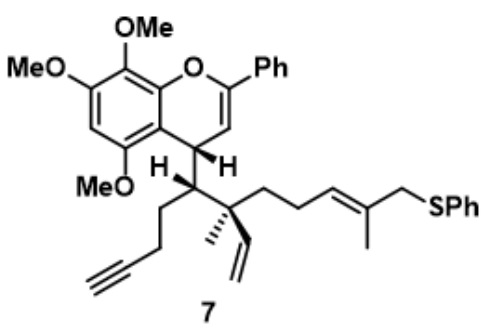


National Center for Organic Mass Spectrometry in Shanghai Shanghai Institute of Organic Chemistry Chinese Academic of Sciences High Resolution MS DATA REPORT

Instrument: Thermo Fisher Scientific LTQ FTICR-MS

Card Serial Number : D182587

Sample Serial Number: NO.34 HJD-X-70

Operator : DONG

Date: $2018 / 09 / 04$

Operation Mode: DART POSITIVE Ion Mode

Elemental composition search on mass 501.30<smiles>C=C1CC[C@@H]([C@H]2C=C(c3ccccc3)Oc3c(OC)cc(OC)c(OC)c32)[C@H]2CC[C@@H](C(=C)C)C[C@H]12</smiles>

$\mathrm{m} / \mathrm{z}=496.30-506.30$

\begin{tabular}{l|l|r|r|l}
$\mathrm{m} / \mathrm{z}$ & $\begin{array}{c}\text { Theo. } \\
\text { Mass }\end{array}$ & $\begin{array}{c}\text { Delta } \\
(\mathrm{ppm})\end{array}$ & \multicolumn{1}{c|}{$\begin{array}{l}\text { RDB } \\
\text { equiv. }\end{array}$} & \multicolumn{1}{c}{ Composition } \\
01.2993 & 501.2997 & -0.76 & -1.0 & $\mathrm{C}_{19} \mathrm{H}_{48} \mathrm{O}_{3} \mathrm{~N}_{7} \mathrm{Br}$ \\
& 501.2999 & -1.33 & 13.5 & $\mathrm{C}_{33} \mathrm{H}_{41} \mathrm{O}_{4}$ \\
\hline & 501.2986 & 1.35 & 14.0 & $\mathrm{C}_{31} \mathrm{H}_{39} \mathrm{O}_{3} \mathrm{~N}_{3}$ \\
& 501.2983 & 1.92 & -0.5 & $\mathrm{C}_{17} \mathrm{H}_{46} \mathrm{O}_{2} \mathrm{~N}_{10} \mathrm{Br}$ \\
& 501.3013 & -4.00 & 18.5 & $\mathrm{C}_{34} \mathrm{H}_{37} \mathrm{~N}_{4}$ \\
& 501.2973 & 4.03 & 14.5 & $\mathrm{C}_{29} \mathrm{H}_{37} \mathrm{O}_{2} \mathrm{~N}_{6}$
\end{tabular}

